\title{
Collaborative Dynamics between Firms and Consumers: an Empirical Review from an Integrated Management Perspective
}

\author{
Eleonora Paolocci ${ }^{1}$ \\ ${ }^{1}$ Research Doctorate in Corporate Communication, IULM University, Milan, Italy \\ Correspondence: Eleonora Paolocci, IULM University, Via Carlo Bò 1, 20143 Milan, Italy. \\ E-mail:eleonora.paolocci@iulm.it
}

Received: March 4, 2014

Accepted: June29, $2014 \quad$ Online Published: July 25, 2014

doi:10.5539/ijbm.v9n8p1

URL: http://dx.doi.org/10.5539/ijbm.v9n8p1

\begin{abstract}
Combining bibliographic and content analysis, the study quantitatively analyses the scientific production on consumer involvement in marketing and innovation processes, a complex challenge for managers. Recently, the attention given to collaborative approaches has grown exponentially and this area of research has been given new impetus especially by the opportunities that virtual environments offer, and which allow firms to transform new interaction modalities into added value. However, there is a lack of literature reviews which rely on recent data. The topic, from an empirical and longitudinal point of view, appears to be under-explored. Covering the period from 1976 to December 2013, findings show broad trends in the reviewed studies and, extrapolating key concepts, highlight the main dimensions that have to be taken into account to explore the subject. The present review shows that a variety of focal points are used and interdisciplinary influences are expected to continue. Suggestions for future research have also been put forward.
\end{abstract}

Keywords: B2C, consumer involvement, literature review, market driven innovation

\section{Introduction}

The importance of collaborating with consumers in the innovation process has been recognized for many years and there has been a steady proliferation of studies on this topic (Von Hippel, 1976, 1978, 1986, 1988; Grönross, 1990; Day, 1991; Gales \& Mansour-Cole, 1995; Bruce, Leverick, Littler, \& Wilson, 1995; Prahalad \& Ramaswamy, 2004a; Vargo \& Lusch, 2004). The concept of cooperation and value co-creation includes a range of potential forms of collaboration: co-conception of ideas, co-design, co-production, co-promotion, co-pricing, co-distribution, co-consumption, co-maintenance, co-disposal, co-outsourcing (Sheth \& Uslay, 2007); co-meaning, co-experience (Frow, Payne, \& Storbacka, 2011); collaborative filtering systems and word of mouth (Adomavicius \& Tuzhilin, 2005; Chen \& Xie, 2008); continuous co-innovation in the various aspects of a company's management, such as approach to the market, competitive positioning, organisational form, products and processes (Prandelli, Verona, \& Raccagni, 2006). The fundamental idea embedded in the strategy of value co-creation is that it should be understood as "the benefits created from helping customers to achieve their desires and aspirations with their products experiences, and as a result of the revenue created from tailoring customer individual requirements" (Romero \& Molina, 2011, p. 11).

According to this perspective, a value co-creation context is characterized by a continuous process of discovery of new sources, new opportunities and ways to co-create it by and for consumers and companies. It integrates firms' competencies and involves consumers' individual preferences into a network for the co-creation of the next level of value for products, services and experiences to be launched on the market.

Also, this new perspective is central in open-business innovation (Chesbrough, 2003, 2006, 2011) and in network-centric innovation (NCI) (Nambisan \& Sawhney, 2007) models. These approaches emphasize the need to continuously experiment around value creation and encourage companies to open up to new ideas coming from the outside, combining them with in-house ideas to develop new products, projects and systems which can then be brought to the market.

In recent years, the attention given to collaborative approaches, based on the emergence of a new creative consumer (Berthon, Pitt, McCarthy, \& Kates, 2007; Berthon, Campbell, Pitt, \& McCarthy, 2011; Jespersen, 2011; Page \& Pitt, 2011; Cova \& Cova, 2012), has grown exponentially. This area of research has been given new 
impetus especially by the rapid growth of the Web, through which organisational learning develops thanks to the continuous interaction with the market and the shared creation of the offer. The Web also serves as a powerful platform to access external and distributed knowledge.

Existing academic literature suggests a significant potential of collaboration with consumers and, in general, with external subjects in the process of market value creation through ICTs (Von Hippel 2001; Von Hippel \& Katz, 2002; Sawhney, Verona, \& Prandelli, 2005; Bilgram, Brem, \& Voigt, 2008; Füller \& Von Hippel, 2008; Prandelli, Sawhney, \& Verona, 2008; Choi \& Cheung, 2008; Füller, Muhlbacher, Matzler, \& Jawecki, 2009; Morgan \& Wang, 2010). Considerable attention has been given to the benefits offered by the advent of digital technologies: low-cost interaction; increase in the speed and duration of the engagement process; easier sharing processes if compared to what can be done offline, where dynamics are limited to contexts of physical closeness (Dahan \&Hauser, 2002; Afuah, 2003); customerisation, that is, maximum strategic flexibility for companies, both in terms of product technological customization and marketing policies (Wind \& Rangaswamy, 2001); taking on board of various suggestions and knowledge inputs coming from the "periphery", which break consolidated patters in innovation processes.

Because of this growth of research, a review of the state of the art in this domain is deemed necessary. The topic deserves particular attention given the opportunities that the Internet offers: the challenge for firms is to exploit and transform new connection and interaction modalities into added value.

However, literature lacks literature reviews which rely on recent data: the topic, from an empirical and longitudinal point of view, appears to be under-explored. In particular, some contributions have a conceptual nature and have been carried out through a narrative review method (see for example: Hoyer, Chandy, Dorotic, Krafft, \&Singh, 2010; Greer \& Lei, 2012) or within the context of the OI general paradigm (see for example: Gianiodis, Ellis, \& Secchi, 2010; Giannopoulou, Yström, \& Ollila, 2011), while other studies use a quantitative review method but mainly focus on specific aspects (see for example: Di Stefano, Gambardella, \& Verona, 2012 on the juxtaposition between technology and demand as sources of innovations).

Given the illustrated context and following the integrated marketing and innovation management perspective suggested by Ofek \& Toubia (2010), the goal of this study is to map the scientific production related to the subject, illustrate the state of the art of research and highlight broad trends in the reviewed studies.

To achieve this aim, an empirical literature review has been carried out (Rumrill, Fitzgerald, \& Merchant, 2010). In terms of methodology, the review process uses a mainly quantitative-descriptive approach, based on a survey of bibliographic scientific-academic sources.

The following set of research questions drive the study:

RQ1. With what frequency has the topic been studied, also in diachronic terms?

RQ2. Which are the most involved academic-disciplinary areas?

RQ3. What are the reference scientific sources?

RQ4. Which are the most prevalent countries?

RQ5. What types of contributions have been produced on the considered topic?

RQ6 a, b. Which are the most recurrent topics in the studies and the most widely used keywords?

\section{RQ7. Which are the most prevalent scientific institutions?}

In addition, to get a sense of the community that has formed around this topic, the author also provides an overview of who has been working in advancing this area of research, identifying the most prolific authors and the type of authorship.

The structure of the paper is as follows: first, the methodology and study design will be illustrated; in the central section, findings and studies related to the investigated phenomenon will be presented. In the last section, results will be discussed. The author also provides insights from the reviewed literature to better understand consumer involvement processes and put forward suggestions for future research.

\section{Review Method}

\subsection{Scope of the Study}

The study mainly takes on the point of view of firms in B2C contexts, given some prerogatives which make their exploration particularly stimulating.

First, studies on collaborative processes were originally carried out mainly in B2B markets, where they boast a 
longer tradition (cf. studies on strategic alliances: Håkansson 1982; Håkansson \& Lundgren, 1995; Håkansson, Ford, Gadde, Snehota, \& Waluszewski, 2009; the development of the concept of customer-supplier interaction, largely explored since the $80 \mathrm{~s}$ by the researchers of the IMP - Industrial Marketing and Purchasing; the study of purchasing patterns in B2B markets: Ford 2004; social media utilization in B2B relationships: Kärkkäinen, Jussila, \&Väisänen, 2013).

Second, organizations, in their role as purchasers, and end customers follow significantly different purchasing and decision-making models and processes. In the latter case, non-rational factors and inputs are more significant purchase drivers, while in the former it is economic incentives which play a major role.

Third, B2C markets contain more elements of risk, due to the size of the scope of reference and the wider language gap, which is linked to the difficulty of directly understanding needs. Of course, the statement by Gummesson and Polese (2009), according to which B2B and B2C markets are both part of a wider, more complex and common context is taken into due account (Note 1).

Consistently with the delimitation of the illustrated research area, the analysis of the existing literature focuses on specific content characteristics. The legal aspects related to the management of intellectual property rights and the appropriability regime of the outputs of collaborative processes were excluded, as well as the problematic nature and unforeseeability of co-creation processes (see for example: Cova, Dalli, \& Zwick, 2011; Echeverri \& Skålen, 2011; Fisher \& Smith, 2011). Similarly, the critical-sociological currents which highlight the work carried out by the new figure of the working consumer (see for example: Cova \& Dalli, 2009; Zwick, Bonsu, \&Darmody, 2008), papers centred on the non-integration of consumers in the market, such as works on "consumer resistance" (Wipperfürt, 2005) and those which analyse the emergence of forms of co-entrepreneurship or tribal entrepreneurship (Cova, Kozinets, \& Shanlar, 2007; Cova \&White, 2010) were also excluded. The approach which, in the context of brand management studies, has developed the concepts of brand valueco-creationmodel (Iglesias, Ind, \& Alfaro, 2013) and multi-stakeholder brand meaning co-creation (Vallaster \& Von Wallpach, 2013) was also left out. By embracing a mainly managerial perspective, the study finally excludes papers produced by academic research on consumption which focus exclusively on the role of consumers in the co-creation of meaning, where value is transferred to the market and becomes accessible by companies, thus emphasizing the productive role of consumers.

\subsection{Sample and Data Collection}

By using a descriptive approach, an empirical analysis of the existing literature was carried out. The object of such analysis was the monitoring of the following bibliographic sources: journal articles, proceedings papers, working papers. The tools used to support the research were the ISI Web of Knowledge (Web of Science) and EBSCO (Business Source Premier) databases, which complement each other.

Books-including e-books and monographs - were excluded from the analysis as the ISI database does not include these types of sources in the direct indexation mode. The time of publication of the analysed research material ranges from 1976 to December 2013. The units of analysis taken into account were the title, the abstract, the keywords provided by the author and the full body of the papers. The research was limited to contributions in English.

The methodological approach included the use of a two-step procedure, so as to identify an exhaustive and relevant set of contributions, and the use of the Semantic search / Keyword-based retrieval and Content analysis (Kassarjian, 1977; Krippendorff, 1980).

Content analysis is a descriptive method based on the observation of communication contents. One of its advantages lies in the fact that it relies on secondary words. This means that it is not prone to the most common methodological mistakes, as the researcher has minimum impact on data. In addition, it can be applied even to large quantities of data. Each article was scanned for appropriate contents. The search, both in individual and aggregate form, was performed with logical or Boolean operators, using adequately selected key words: co-creation, co-creation, co-design, collaborative innovation, collaboration policy, consumer engagement, consumer empowerment, consumer feedback, consumer insights, consumer involvement, contest, crowd sourcing, demand pull innovation, idea generation, online consumer panel, market driven innovation, open innovation, user innovation.

After removing all duplicates-both from the output data individually produced by each of the databases and those derived from crossing the two tools-the results of the first research stage were analysed based on the mentioned key words. 789 articles were identified. Drawing from the sample of studies from the first stage, the search process then adopted more specific boundary criteria for the review. The following were therefore 
excluded: contributions which focus solely on B2B contexts and intercompany partnerships; research which specifically focuses on the consumer-individual as a privileged perspective, such as socio-psychological studies on the motivations which push the subject to cooperation.

The final output is a list made available to other researchers to build upon, which includes 118 contributions (115 journal papers, 2 working papers, 1 conference proceeding). Table 1 presents this selection. For each identified study, the following data have been highlighted: year of publication; academic area / research setting (indicating the main reference field and stream of literature to which the research belongs); author; source; ranking (where applicable); topic / focus (summarizing the central idea of the contribution); author-provided keywords; main findings / insights (summarizing the primary conclusions of the study and the insights provided); scientific institution; country; type of study.

Each paper was read and coded as belonging to the most appropriate categories. In the study, the process of content analysis coding was complemented by open coding. The method of open coding followed specific steps. First, each paper was read in order to check for relevance and actual fit in the initial categorization resulting from the structured coding. In particular, with reference to the "topic / focus" class, when a coder did not identify an appropriate existing subcategory, new categorizations were reconsidered after the preliminary round of coding. Articles for which new classifications had been suggested were rotated again and reconsidered for recoding.

Table 1. Literature review

\begin{tabular}{|c|c|c|c|c|c|c|c|c|c|c|}
\hline Year & $\begin{array}{l}\text { Academic } \\
\text { area } \\
\text { /research } \\
\text { setting }\end{array}$ & Author & Source & $\begin{array}{l}\text { Ranking } \\
\text { (where } \\
\text { applicable) } \\
{ }^{*} \text { IF-ISI } \\
\text { (2012) } \\
\end{array}$ & Topic / Focus & $\begin{array}{l}\text { Author-provided } \\
\text { keywords }\end{array}$ & $\begin{array}{l}\text { Highlights / Main } \\
\text { findings / Insights }\end{array}$ & $\begin{array}{l}\text { Scientific } \\
\text { Institution }\end{array}$ & Country & Type of study \\
\hline 2013 & $\begin{array}{l}\text { Innovation } \\
\text { Management / } \\
\text { NPD }\end{array}$ & $\begin{array}{l}\text { Hienerth C., } \\
\text { von Hippel E., } \\
\text { Jensen M.B. }\end{array}$ & Research Policy & 2.850 & $\begin{array}{l}\text { Efficiency of user vs. } \\
\text { producer innovation in } \\
\text { the whitewater kayaking } \\
\text { industry }\end{array}$ & $\begin{array}{l}\text { user innovation, } \\
\text { efficiency } \\
\text { measurement, } \\
\text { industry } \\
\text { development }\end{array}$ & $\begin{array}{l}\text { User consumers are } \\
\text { more prolific and } \\
\text { efficient product } \\
\text { developers than } \\
\text { producers in the } \\
\text { early stages of the } \\
\text { field; producers get } \\
\text { more efficient as } \\
\text { the field matures }\end{array}$ & $\begin{array}{l}\text { WHU Otto } \\
\text { Beisheim School } \\
\text { of Management; } \\
\text { MIT Sloan } \\
\text { School of } \\
\text { Management; } \\
\text { Department of } \\
\text { Economics and } \\
\text { Business, Aarhus } \\
\text { University }\end{array}$ & $\begin{array}{l}\text { Germany, } \\
\text { USA, } \\
\text { Denmark }\end{array}$ & $\begin{array}{l}\text { Empirical paper } \\
\text { (mixed } \\
\text { approach: } \\
\text { qualitative- } \\
\text { explorative } \\
\text { interviews-, } \\
\text { quantitative- } \\
\text { multiple } \\
\text { surveys-) }\end{array}$ \\
\hline 2013 & $\begin{array}{l}\text { Marketing } \\
\text { management }\end{array}$ & $\begin{array}{l}\text { Djelassi S., } \\
\text { Decoopman I. }\end{array}$ & $\begin{array}{l}\text { Industrial } \\
\text { Marketing } \\
\text { Management }\end{array}$ & 1.933 & $\begin{array}{l}\text { Customers' participation } \\
\text { in PD processes through } \\
\text { crowdsourcing practices }\end{array}$ & $\begin{array}{l}\text { open business } \\
\text { model; } \\
\text { crowdsourcing; } \\
\text { value co-creation; } \\
\text { customer } \\
\text { participation }\end{array}$ & $\begin{array}{l}\text { Modeling of an } \\
\text { open business } \\
\text { model based on } \\
\text { crowdsourcing }\end{array}$ & $\begin{array}{l}\text { SKEMA } \\
\text { Business School, } \\
\text { University of } \\
\text { Lille Nord de } \\
\text { France }\end{array}$ & France & $\begin{array}{l}\text { Empirical paper } \\
\text { (multiple case } \\
\text { study) }\end{array}$ \\
\hline 2013 & $\begin{array}{l}\text { Technology } \\
\text { and } \\
\text { Innovation } \\
\text { Management }\end{array}$ & $\begin{array}{l}\text { Goduscheit } \\
\text { R.C., } \\
\text { Jørgensen J.H. }\end{array}$ & $\begin{array}{l}\text { International } \\
\text { Journal of } \\
\text { Technology } \\
\text { Management }\end{array}$ & 0.564 & $\begin{array}{l}\text { Review of the literature } \\
\text { on toolkits for user } \\
\text { innovation }\end{array}$ & $\begin{array}{l}\text { customer-active } \\
\text { paradigm, } \\
\text { literature review, } \\
\text { user toolkits for } \\
\text { innovation }\end{array}$ & $\begin{array}{l}\text { The user toolkit for } \\
\text { innovation } \\
\text { approach } \\
\text { represents a } \\
\text { distinct theoretical } \\
\text { position in the } \\
\text { literature, while the } \\
\text { empirical evidence } \\
\text { is closer to other } \\
\text { approaches within } \\
\text { the user innovation } \\
\text { paradigm like } \\
\text { customization }\end{array}$ & $\begin{array}{l}\text { Integrative } \\
\text { Innovation } \\
\text { Management } \\
\text { Unit, University } \\
\text { of Southern } \\
\text { Denmark }\end{array}$ & Denmark & $\begin{array}{l}\text { Conceptual/ } \\
\text { theoretical } \\
\text { paper }\end{array}$ \\
\hline 2013 & Marketing & $\begin{array}{l}\text { Syam N.B., } \\
\text { Pazgal A. }\end{array}$ & $\begin{array}{l}\text { Marketing } \\
\text { Science }\end{array}$ & 2.201 & $\begin{array}{l}\text { Co-Creation } \\
\text { environments where te } \\
\text { price mechanism is } \\
\text { crucial in mediating the } \\
\text { value exchange process }\end{array}$ & $\begin{array}{l}\text { co-creation, } \\
\text { customization, } \\
\text { externalities, game } \\
\text { theory, networks }\end{array}$ & $\begin{array}{l}\text { Mathematical } \\
\text { modellization of } \\
\text { co-creation } \\
\text { between firms and } \\
\text { consumers as a } \\
\text { network of } \\
\text { externalities. } \\
\text { A monopoly firm } \\
\text { opts for co-creation } \\
\text { with customers }\end{array}$ & $\begin{array}{lr}\text { C. T. } & \text { Bauer } \\
\text { College } & \text { of } \\
\text { Business, } \\
\text { University of } \\
\text { Houston; } \\
\text { Jones Graduate } \\
\text { School of } \\
\text { Business, Rice } \\
\text { University }\end{array}$ & USA & $\begin{array}{l}\text { Methodological } \\
\text { paper (analytic } \\
\text { method) }\end{array}$ \\
\hline
\end{tabular}




\begin{tabular}{|c|c|c|c|c|c|c|c|c|c|c|}
\hline & & & & & & & $\begin{array}{l}\text { rather than deal } \\
\text { with passive } \\
\text { price-taking } \\
\text { consumers: the } \\
\text { pricing affects } \\
\text { customers' } \\
\text { incentive to invest } \\
\text { effort in the } \\
\text { innovation-product } \\
\text { ion stage }\end{array}$ & & & \\
\hline 2013 & $\begin{array}{l}\text { Technology } \\
\text { and } \\
\text { Innovation } \\
\text { Management }\end{array}$ & $\begin{array}{l}\text { Christiansen } \\
\text { J.K., Gasparin } \\
\text { M., Varnes } \\
\text { C.J. }\end{array}$ & $\begin{array}{l}\text { Research } \\
\text { Technology } \\
\text { Management }\end{array}$ & 0.712 & $\begin{array}{l}\text { Improving Design with } \\
\text { Open Innovation }\end{array}$ & $\begin{array}{l}\text { adaptation, } \\
\text { innovation } \\
\text { management } \\
\text { technologies, open } \\
\text { innovation, } \\
\text { sensemaking }\end{array}$ & $\begin{array}{l}\text { Innovation } \\
\text { develops in } \\
\text { nonlinear ways. } \\
\text { Firms involved in } \\
\text { OI need a broad } \\
\text { knowledge of the } \\
\text { various potential } \\
\text { elements of an OI } \\
\text { effort and a flexible } \\
\text { attitude toward } \\
\text { their application }\end{array}$ & $\begin{array}{l}\text { Copenhagen } \\
\text { Business School }\end{array}$ & Denmark & $\begin{array}{l}\text { Empirical paper } \\
\text { (single case } \\
\text { study) }\end{array}$ \\
\hline 2013 & $\begin{array}{l}\text { Technology } \\
\text { and } \\
\text { Innovation } \\
\text { Management }\end{array}$ & $\begin{array}{l}\text { Kosonen, M., } \\
\text { Gan, C., } \\
\text { Olander, H., \& } \\
\text { Blomqvist, K. }\end{array}$ & $\begin{array}{l}\text { International } \\
\text { Journal of } \\
\text { Innovation } \\
\text { Management }\end{array}$ & - & $\begin{array}{l}\text { User driven innovation } \\
\text { in online communities }\end{array}$ & $\begin{array}{l}\text { crowdsourcing, } \\
\text { institutional trust, } \\
\text { knowledge } \\
\text { sharing, norms, } \\
\text { online community, } \\
\text { trust }\end{array}$ & $\begin{array}{l}\text { Importance of } \\
\text { providing } \\
\text { continued support } \\
\text { for knowledge } \\
\text { integration, such as } \\
\text { more opportunities } \\
\text { for interaction and } \\
\text { constructive } \\
\text { feedback }\end{array}$ & $\begin{array}{l}\text { Lappeenranta } \\
\text { University of } \\
\text { Technology; } \\
\text { School of } \\
\text { Information } \\
\text { Management Sun } \\
\text { Yat-sen } \\
\text { University }\end{array}$ & $\begin{array}{l}\text { Finland, } \\
\text { China }\end{array}$ & $\begin{array}{l}\text { Empirical paper } \\
\text { (quantitative } \\
\text { approach: } \\
\text { survey) }\end{array}$ \\
\hline 2013 & $\begin{array}{l}\text { Innovation } \\
\text { Management/ } \\
\text { NPD }\end{array}$ & Bayus B.L. & $\begin{array}{l}\text { Management } \\
\text { Science }\end{array}$ & 1.859 & $\begin{array}{l}\text { Crowdsourcing } \text { to } \\
\text { collect new product } \\
\text { ideas }\end{array}$ & $\begin{array}{l}\text { creativity, fixation, } \\
\text { ideation, } \\
\text { innovation, } \\
\text { marketing }\end{array}$ & $\begin{array}{l}\text { Highlighting of the } \\
\text { challenges in } \\
\text { maintaining an } \\
\text { ongoing supply of } \\
\text { quality ideas from } \\
\text { the crowd over } \\
\text { time }\end{array}$ & $\begin{array}{l}\text { Kenan-Flagler } \\
\text { Business School, } \\
\text { University of } \\
\text { North Carolina }\end{array}$ & USA & $\begin{array}{l}\text { Empirical paper } \\
\text { (single case } \\
\text { study) }\end{array}$ \\
\hline 2013 & $\begin{array}{l}\text { Business } \\
\text { research }\end{array}$ & $\begin{array}{ll}\text { Gebauer } & \text { J., } \\
\text { Füller } & \text { J., } \\
\text { Pezzei R. } & \end{array}$ & $\begin{array}{l}\text { Journal of } \\
\text { Business } \\
\text { Research }\end{array}$ & 1.484 & $\begin{array}{l}\text { Dynamics of online } \\
\text { innovation communities }\end{array}$ & $\begin{array}{l}\text { co-creation, online } \\
\text { innovation } \\
\text { communities, } \\
\text { dysfunctional } \\
\text { behavior, } \\
\text { word-of-mouth, } \\
\text { fairness, sense of } \\
\text { community }\end{array}$ & $\begin{array}{l}\text { Managing possible } \\
\text { criticality in } \\
\text { co-creation and } \\
\text { innovation } \\
\text { communities needs } \\
\text { open dialog, } \\
\text { co-negotiation and } \\
\text { co-moderation by } \\
\text { the firm }\end{array}$ & $\begin{array}{l}\text { Innsbruck } \\
\text { University } \\
\text { School of } \\
\text { Management }\end{array}$ & Austria & $\begin{array}{l}\text { Empirical paper } \\
\text { (single case } \\
\text { study) }\end{array}$ \\
\hline 2013 & $\begin{array}{l}\text { Technology } \\
\text { and } \\
\text { Innovation } \\
\text { Management }\end{array}$ & $\begin{array}{l}\text { Parmentier G., } \\
\text { Gandia R. }\end{array}$ & $\begin{array}{l}\text { Creativity and } \\
\text { Innovation } \\
\text { Management }\end{array}$ & 0.855 & $\begin{array}{l}\text { User toolkit role in the } \\
\text { sustainable innovative } \\
\text { approach with a } \\
\text { community }\end{array}$ & not provided & $\begin{array}{l}\text { Toolkit as a way to } \\
\text { manage: boundary } \\
\text { between firm and } \\
\text { users; innovation } \\
\text { of an existing } \\
\text { product through a } \\
\text { sustainable } \\
\text { relationship with } \\
\text { users }\end{array}$ & $\begin{array}{l}\text { University of } \\
\text { Grenoble; } \\
\text { INSEEC } \\
\text { Business School }\end{array}$ & France & $\begin{array}{l}\text { Empirical paper } \\
\text { (single case } \\
\text { study) }\end{array}$ \\
\hline 2013 & $\begin{array}{l}\text { Marketing } \\
\text { management }\end{array}$ & $\begin{array}{ll}\text { Ind } & \text { N., } \\
\text { Iglesias } & \text { O., } \\
\text { Schultz M. }\end{array}$ & $\begin{array}{l}\text { California } \\
\text { Management } \\
\text { Review }\end{array}$ & 1.667 & $\begin{array}{l}\text { Understanding how } \\
\text { consumer participation } \\
\text { in virtual co-creation } \\
\text { projects emerges and } \\
\text { develops }\end{array}$ & not provided & $\begin{array}{l}\text { The first } \\
\text { requirement for a } \\
\text { successful online } \\
\text { co-creation } \\
\text { environment is to } \\
\text { facilitate and to } \\
\text { encourage social } \\
\text { conversations } \\
\text { among people }\end{array}$ & $\begin{array}{l}\text { Oslo School of } \\
\text { Management; } \\
\text { ESADE } \\
\text { Universitat } \\
\text { Ramon Llull; } \\
\text { Copenhagen } \\
\text { Business School }\end{array}$ & $\begin{array}{l}\text { Norway, } \\
\text { Spain, } \\
\text { Denmark }\end{array}$ & $\begin{array}{l}\text { Methodological } \\
\text { paper }\end{array}$ \\
\hline 2013 & Management & $\begin{array}{l}\text { King A., } \\
\text { Lakhani K.R. }\end{array}$ & $\begin{array}{l}\text { MIT Sloan } \\
\text { management } \\
\text { review }\end{array}$ & 1.413 & $\begin{array}{l}\text { OI and customer } \\
\text { feedback to identify } \\
\text { ideas: the emergence of }\end{array}$ & not provided & $\begin{array}{l}\text { Problems created } \\
\text { by openness: with } \\
\text { OI, the firm pays }\end{array}$ & $\begin{array}{lr}\text { Dartmouth } \\
\text { College's } \\
\text { School }\end{array} \begin{array}{r}\text { of } \\
\end{array}$ & USA & $\begin{array}{l}\text { Empirical paper } \\
\text { (mixed } \\
\text { approach: }\end{array}$ \\
\hline
\end{tabular}




\begin{tabular}{|c|c|c|c|c|c|c|c|c|c|c|c|}
\hline & & & & & & $\begin{array}{l}\text { distributed-innovation } \\
\text { forms (communities, OI } \\
\text { platforms, contests) }\end{array}$ & & $\begin{array}{l}\text { for a design only } \\
\text { after it has been } \\
\text { completed, so it } \\
\text { should consider } \\
\text { mechanisms for } \\
\text { lowering cost of } \\
\text { participation: } \\
\text { provide contestants } \\
\text { with design tools, } \\
\text { break problems } \\
\text { into smaller pieces, } \\
\text { each with a prize; } \\
\text { while outsiders } \\
\text { may have unique } \\
\text { insights, their } \\
\text { concepts are not } \\
\text { always aligned } \\
\text { with the firm's one: } \\
\text { retain explicit } \\
\text { residual control to } \\
\text { exercise over the } \\
\text { designs; } 3 \text {. the } \\
\text { more dispersed the } \\
\text { skills or the more } \\
\text { uncertainty about } \\
\text { what skills are } \\
\text { needed, the more } \\
\text { valuable opening } \\
\text { will be }\end{array}$ & $\begin{array}{l}\text { Business; } \\
\text { Harvard } \\
\text { Business School }\end{array}$ & & $\begin{array}{l}\text { quantitative- } \\
\text { multiple } \\
\text { surveys-, } \\
\text { qualitative- } \\
\text { field } \\
\text { experiment-) }\end{array}$ \\
\hline 2013 & $\begin{array}{l}\text { Technology } \\
\text { and Society }\end{array}$ & $\begin{array}{l}\text { Godin } \\
\text { Lane J.P. }\end{array}$ & B., & $\begin{array}{l}\text { Science, } \\
\text { Technology \& } \\
\text { Human Values }\end{array}$ & 2.406 & $\begin{array}{l}\text { Demand-pull model of } \\
\text { innovation }\end{array}$ & $\begin{array}{l}\text { demand-pull } \\
\text { model, innovation } \\
\text { policy, innovation } \\
\text { studies, linear } \\
\text { model of } \\
\text { innovation, } \\
\text { science, supply of } \\
\text { inventions, } \\
\text { technological } \\
\text { innovation, } \\
\text { technology }\end{array}$ & $\begin{array}{l}\text { The concept of } \\
\text { demand as a factor } \\
\text { explaining } \\
\text { technological } \\
\text { innovation } \\
\text { emerged in the } \\
\text { 1960s, was } \\
\text { formalized into } \\
\text { models in the } \\
\text { 1970-1980s, then } \\
\text { got integrated into } \\
\text { "multidimensional } \\
\text { " models. From } \\
\text { then on, the } \\
\text { demand-pull model } \\
\text { disappeared from } \\
\text { the literature, } \\
\text { existing only as an } \\
\text { object of the past, } \\
\text { like the linear } \\
\text { model of } \\
\text { innovation }\end{array}$ & $\begin{array}{l}\text { INRS University } \\
\text { (Institut national } \\
\text { de la recherche } \\
\text { scientifique); } \\
\text { University at } \\
\text { Buffalo }\end{array}$ & $\begin{array}{l}\text { Canada, } \\
\text { USA }\end{array}$ & $\begin{array}{l}\text { Conceptual/ } \\
\text { theoretical } \\
\text { paper }\end{array}$ \\
\hline 2013 & $\begin{array}{l}\text { Technology } \\
\text { and } \\
\text { Innovation } \\
\text { Management }\end{array}$ & $\begin{array}{l}\text { Porter } \\
\text { Devaraj } \\
\text { Sun D. }\end{array}$ & $\begin{array}{l}\text { C.E., } \\
\text { S.S., }\end{array}$ & $\begin{array}{l}\text { Journal of } \\
\text { Management } \\
\text { Information } \\
\text { Systems }\end{array}$ & 1.262 & $\begin{array}{l}\text { Value creation in virtual } \\
\text { communities: } \\
\text { customer-initiated vs } \\
\text { firm-sponsored model }\end{array}$ & $\begin{array}{l}\text { attribution theory, } \\
\text { co-creation, online } \\
\text { communities, } \\
\text { online trust, } \\
\text { user-generated } \\
\text { content, virtual } \\
\text { communities }\end{array}$ & $\begin{array}{l}\text { Results suggest a } \\
\text { continuum of value } \\
\text { creation whereby } \\
\text { firms extract } \\
\text { greater value as } \\
\text { they migrate } \\
\text { toward the } \\
\text { firm-sponsored } \\
\text { model. This } \\
\text { strategy has a } \\
\text { positive, direct } \\
\text { effect on the } \\
\text { trust-building } \\
\text { process }\end{array}$ & $\begin{array}{l}\text { Jones Graduate } \\
\text { School of } \\
\text { Business, Rice } \\
\text { University; } \\
\text { Mendoza } \\
\text { College } \\
\text { Business, of } \\
\text { University of } \\
\text { Notre Dame }\end{array}$ & USA & $\begin{array}{l}\text { Empirical paper } \\
\text { (quantitative } \\
\text { approach } \\
\text {-survey-) }\end{array}$ \\
\hline 2013 & $\begin{array}{l}\text { Marketing } \\
\text { management }\end{array}$ & Bin Guo & & $\begin{array}{l}\text { Industrial } \\
\text { Marketing }\end{array}$ & 1.933 & $\begin{array}{l}\text { Driving factors of user } \\
\text { innovation }\end{array}$ & $\begin{array}{l}\text { user innovation, } \\
\text { user innovator, }\end{array}$ & $\begin{array}{l}\text { Integrative model } \\
\text { of user innovation, }\end{array}$ & $\begin{array}{l}\text { School of } \\
\text { Management, }\end{array}$ & China & $\begin{array}{l}\text { Empirical paper } \\
\text { (mixed }\end{array}$ \\
\hline
\end{tabular}




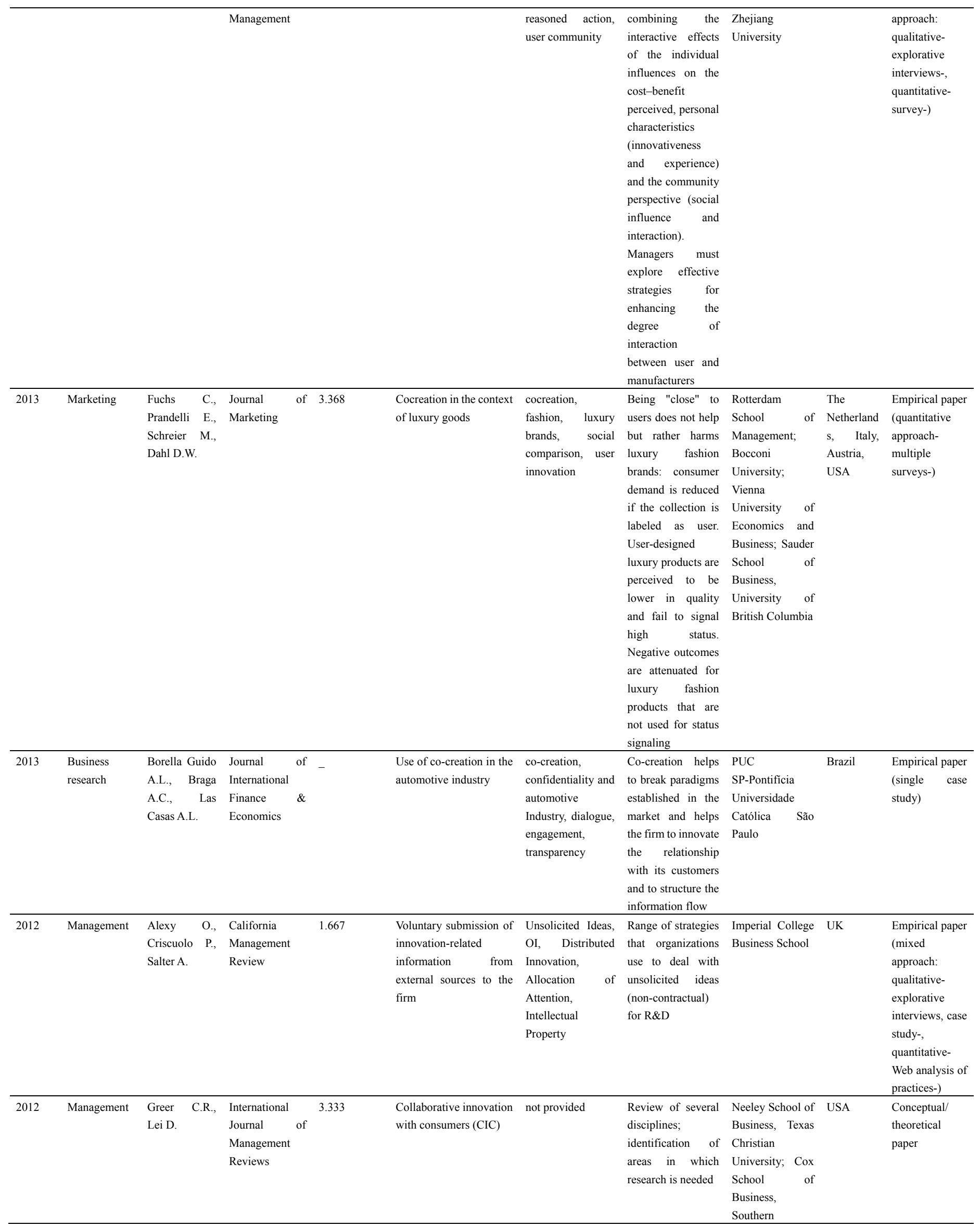




\begin{tabular}{|c|c|c|c|c|c|c|c|c|c|c|}
\hline & & & & & & & & $\begin{array}{l}\text { Methodist } \\
\text { University }\end{array}$ & & \\
\hline 2012 & $\begin{array}{l}\text { Organization } \\
\text { Management }\end{array}$ & $\begin{array}{ll}\text { Keinz } & \text { P., } \\
\text { Hienerth } & \text { C., } \\
\text { Lettl C. } & \end{array}$ & $\begin{array}{l}\text { Journal of } \\
\text { Organization } \\
\text { Design }\end{array}$ & - & $\begin{array}{l}\text { Implications of user } \\
\text { innovation strategies on } \\
\text { key dimensions of } \\
\text { organizational design }\end{array}$ & $\begin{array}{l}\text { user innovation, } \\
\text { organization } \\
\text { design }\end{array}$ & $\begin{array}{l}\text { Integrated } \\
\text { perspective of the } \\
\text { research fields of } \\
\text { user innovation and } \\
\text { organizational } \\
\text { design }\end{array}$ & $\begin{array}{l}\text { WU Vienna } \\
\text { University of } \\
\text { Economics and } \\
\text { Business; WHU - } \\
\text { Otto Beisheim } \\
\text { School of } \\
\text { Management } \\
\end{array}$ & $\begin{array}{l}\text { Austria, } \\
\text { Germany }\end{array}$ & $\begin{array}{l}\text { Conceptual/ } \\
\text { theoretical } \\
\text { paper }\end{array}$ \\
\hline 2012 & $\begin{array}{l}\text { Technology } \\
\text { and } \\
\text { Innovation } \\
\text { Management }\end{array}$ & $\begin{array}{l}\text { Chesbrough } \\
\text { H. }\end{array}$ & $\begin{array}{l}\text { Research } \\
\text { Technology } \\
\text { Management }\end{array}$ & 0.712 & $\begin{array}{ll}\text { Open } & \text { Innovation } \\
\text { paradigm } & \end{array}$ & $\begin{array}{l}\text { Business model } \\
\text { innovation, } \\
\text { innovation } \\
\text { communities, } \\
\text { intellectual } \\
\text { property, OI }\end{array}$ & $\begin{array}{l}\text { Review of the } \\
\text { recent history of } \\
\text { the OI concept; } \\
\text { perspective on } \\
\text { where OI is going } \\
\text { in the future }\end{array}$ & $\begin{array}{l}\text { Haas School of } \\
\text { Business, } \\
\text { University of } \\
\text { California-Berke } \\
\text { ley }\end{array}$ & USA & $\begin{array}{l}\text { Conceptual/ } \\
\text { theoretical } \\
\text { paper }\end{array}$ \\
\hline 2012 & $\begin{array}{l}\text { Technology } \\
\text { and } \\
\text { Innovation } \\
\text { Management }\end{array}$ & $\begin{array}{l}\text { Di Stefano G., } \\
\text { Gambardella } \\
\text { A., Verona G. }\end{array}$ & Research Policy & 2.850 & $\begin{array}{lr}\text { Demand } & \text { pull } \\
\text { perspectives } & \text { in } \\
\text { innovation studies } & \end{array}$ & $\begin{array}{l}\text { technology push, } \\
\text { demand pull } \\
\text { innovation, } \\
\text { bibliometrics, } \\
\text { co-citation } \\
\text { analysis }\end{array}$ & $\begin{array}{l}\text { Understanding of } \\
\text { how technology } \\
\text { and demand } \\
\text { interact and the } \\
\text { role of demand as a } \\
\text { source of } \\
\text { innovation and } \\
\text { entrepreneurial } \\
\text { opportunities }\end{array}$ & $\begin{array}{l}\text { HEC Paris, } \\
\text { Department of } \\
\text { Strategy and } \\
\text { Business Policy; } \\
\text { Bocconi } \\
\text { University }\end{array}$ & $\begin{array}{l}\text { France, } \\
\text { Italy }\end{array}$ & $\begin{array}{l}\text { Conceptual/ } \\
\text { theoretical } \\
\text { paper }\end{array}$ \\
\hline 2012 & $\begin{array}{l}\text { Technology } \\
\text { and } \\
\text { Information } \\
\text { Management }\end{array}$ & $\begin{array}{l}\text { Chen L., } \\
\text { Marsden J.R., } \\
\text { Zhang Z. }\end{array}$ & $\begin{array}{l}\text { Journal of } \\
\text { Management } \\
\text { Information } \\
\text { Systems }\end{array}$ & 1.262 & $\begin{array}{l}\text { Company-Sponsored } \\
\text { Online Co-Creation } \\
\text { Brainstorming }(\mathrm{COCB})\end{array}$ & $\begin{array}{l}\text { brainstorming, } \\
\text { co-creation, } \\
\text { contribution } \\
\text { quality, sustained } \\
\text { participation }\end{array}$ & $\begin{array}{l}\text { The sponsoring } \\
\text { firms should } \\
\text { develop efficient } \\
\text { processes for } \\
\text { reviewing and } \\
\text { responding to } \\
\text { submitted ideas }\end{array}$ & $\begin{array}{l}\text { School of } \\
\text { Business and } \\
\text { Economics, } \\
\text { Fayetteville State } \\
\text { University; } \\
\text { University of } \\
\text { Connecticut } \\
\end{array}$ & USA & $\begin{array}{l}\text { Empirical paper } \\
\text { (single case } \\
\text { study) }\end{array}$ \\
\hline 2012 & $\begin{array}{l}\text { Innovation } \\
\text { Management/ } \\
\text { NPD }\end{array}$ & $\begin{array}{l}\text { Poetz M.K., } \\
\text { Schreier M. }\end{array}$ & $\begin{array}{l}\text { Journal of } \\
\text { Product } \\
\text { Innovation } \\
\text { Management }\end{array}$ & 1.572 & $\begin{array}{l}\text { Users vs professionals in } \\
\text { generating NP ideas }\end{array}$ & not provided & $\begin{array}{l}\text { Crowdsourcing } \\
\text { might constitute a } \\
\text { promising method } \\
\text { to gather ideas, in } \\
\text { terms of novelty } \\
\text { and customer } \\
\text { benefit, that can } \\
\text { complement those } \\
\text { of a firm }\end{array}$ & $\begin{array}{l}\text { Copenhagen } \\
\text { Business School; } \\
\text { Bocconi } \\
\text { University }\end{array}$ & $\begin{array}{l}\text { Denmark, } \\
\text { Italy }\end{array}$ & $\begin{array}{l}\text { Empirical paper } \\
\text { (single case } \\
\text { study) }\end{array}$ \\
\hline 2012 & $\begin{array}{l}\text { Technology } \\
\text { and } \\
\text { Innovation } \\
\text { Management }\end{array}$ & $\begin{array}{l}\text { Schweitzer } \\
\text { F.M., } \\
\text { Buchinger W., } \\
\text { Gassmann O., } \\
\text { Obrist M. }\end{array}$ & $\begin{array}{l}\text { Research } \\
\text { Technology } \\
\text { Management }\end{array}$ & 0.712 & $\begin{array}{l}\text { Leveraging innovation } \\
\text { through online idea } \\
\text { competitions vs } \\
\text { traditional focus groups }\end{array}$ & $\begin{array}{l}\text { focus groups, } \\
\text { fuzzy front end, } \\
\text { idea competitions, } \\
\text { virtual } \\
\text { communities }\end{array}$ & $\begin{array}{l}\text { Online idea } \\
\text { competitions lead } \\
\text { to more and better } \\
\text { ideas at a lower } \\
\text { cost per idea, while } \\
\text { focus groups yield } \\
\text { richer interactions } \\
\text { with users }\end{array}$ & $\begin{array}{l}\text { Upper Austria } \\
\text { University of } \\
\text { Applied } \\
\text { Sciences; } \\
\text { Institute of } \\
\text { Technology } \\
\text { Management, } \\
\text { University of St. } \\
\text { Gallen; } \\
\text { Newcastle } \\
\text { University }\end{array}$ & $\begin{array}{l}\text { Austria, } \\
\text { Switzerlan } \\
\text { d, UK }\end{array}$ & $\begin{array}{l}\text { Empirical paper } \\
\text { (single case } \\
\text { study) }\end{array}$ \\
\hline 2012 & $\begin{array}{l}\text { Technology } \\
\text { and } \\
\text { Innovation } \\
\text { Management }\end{array}$ & $\begin{array}{l}\text { Leminen S., } \\
\text { Westerlund } \\
\text { M., Nyström } \\
\text { A.G. }\end{array}$ & $\begin{array}{l}\text { Technology } \\
\text { Innovation } \\
\text { Management } \\
\text { Review }\end{array}$ & - & $\begin{array}{l}\text { User-driven living labs } \\
\text { as OI tools to create } \\
\text { value }\end{array}$ & $\begin{array}{l}\text { co-creation, } \\
\text { innovation, living } \\
\text { labs, networks, OI }\end{array}$ & $\begin{array}{l}\text { Living labs are } \\
\text { environments in } \\
\text { which user } \\
\text { experiences reveal } \\
\text { future directions of } \\
\text { PD, strategic tools } \\
\text { to collect data on } \\
\text { trends, competitors } \\
\text { and to support the } \\
\text { firm's business in } \\
\text { short and long term }\end{array}$ & $\begin{array}{l}\text { Aalto University } \\
\text { School of } \\
\text { Business; } \\
\text { Carleton } \\
\text { University's } \\
\text { Sprott School of } \\
\text { Business; School } \\
\text { of Business and } \\
\text { Economics, Åbo } \\
\text { Akademi } \\
\text { University } \\
\end{array}$ & $\begin{array}{l}\text { Canada, } \\
\text { Finland }\end{array}$ & $\begin{array}{l}\text { Empirical paper } \\
\text { (quantitative } \\
\text { approach: } \\
\text { survey) }\end{array}$ \\
\hline 2012 & $\begin{array}{l}\text { Innovation } \\
\text { Management }\end{array}$ & $\begin{array}{l}\text { Bogers M., } \\
\text { West J. }\end{array}$ & $\begin{array}{l}\text { Creativity and } \\
\text { Innovation } \\
\text { Management }\end{array}$ & 0.855 & $\begin{array}{l}\text { Vertically integrated vs } \\
\text { distributed models of } \\
\text { innovation }\end{array}$ & not provided & $\begin{array}{l}\text { Framework for the } \\
\text { strategic } \\
\text { management of } \\
\text { distributed } \\
\text { innovation, }\end{array}$ & $\begin{array}{l}\text { University of } \\
\text { Southern } \\
\text { Denmark; Keck } \\
\text { Graduate } \\
\text { Institute } \\
\end{array}$ & $\begin{array}{l}\text { Denmark, } \\
\text { USA }\end{array}$ & $\begin{array}{l}\text { Conceptual/ } \\
\text { theoretical } \\
\text { paper }\end{array}$ \\
\hline
\end{tabular}




\begin{tabular}{|c|c|c|c|c|c|c|c|c|c|c|}
\hline & & & & & & & $\begin{array}{l}\text { considering } \\
\text { differences in its } \\
\text { features }\end{array}$ & $\begin{array}{l}\text { Applied } \\
\text { Sciences }\end{array}$ & & \\
\hline 2012 & $\begin{array}{l}\text { Technology } \\
\text { and } \\
\text { Innovation } \\
\text { Management }\end{array}$ & $\begin{array}{lc}\text { Füller } & \text { J., } \\
\text { Matzler } & \text { K., } \\
\text { Hutter } & \text { K., } \\
\text { Hautz J. } & \end{array}$ & $\begin{array}{l}\text { Creativity and } \\
\text { Innovation } \\
\text { Management }\end{array}$ & 0.855 & $\begin{array}{l}\text { Consumer' } \\
\text { characteristics for OI } \\
\text { projects }\end{array}$ & not provided & $\begin{array}{lr}\begin{array}{l}\text { Only when } \\
\text { consumers }\end{array} \\
\text { qualified are } \\
\text { motivated and } \\
\text { contribute } \\
\text { promising ideas } \\
\text { and relevant } \\
\text { know-how they are } \\
\text { able to add value to } \\
\text { a producer's } \\
\text { innovation process }\end{array}$ & $\begin{array}{l}\text { Innsbruck } \\
\text { University } \\
\text { School of } \\
\text { Management }\end{array}$ & Austria & $\begin{array}{l}\text { Empirical paper } \\
\text { (quantitative } \\
\text { approach: } \\
\text { survey) }\end{array}$ \\
\hline 2012 & $\begin{array}{l}\text { Marketing } \\
\text { management }\end{array}$ & $\begin{array}{l}\text { Schreier M., } \\
\text { Fuchs C., } \\
\text { Dahl D.W. }\end{array}$ & $\begin{array}{l}\text { Journal of } \\
\text { Marketing }\end{array}$ & 3.368 & $\begin{array}{l}\text { Innovation effect of user } \\
\text { design }\end{array}$ & $\begin{array}{l}\text { co-creation, } \\
\text { innovation, } \\
\text { product design, } \\
\text { user design, user } \\
\text { innovation }\end{array}$ & $\begin{array}{l}\text { Common design by } \\
\text { users enhances } \\
\text { perceptions of a } \\
\text { firm's innovation } \\
\text { ability, leads to } \\
\text { positive outcomes } \\
\text { to purchase and to } \\
\text { recommend the } \\
\text { firm to others. } \\
\text { Number of users, } \\
\text { diversity of } \\
\text { background, lack } \\
\text { of company } \\
\text { constraints, the fact } \\
\text { that consumer } \\
\text { actually uses the } \\
\text { designed product } \\
\text { contribute in } \\
\text { building positive } \\
\text { perceptions }\end{array}$ & $\begin{array}{l}\text { WU Vienna } \\
\text { University of } \\
\text { Economics and } \\
\text { Business; } \\
\text { Rotterdam } \\
\text { School of } \\
\text { Management; } \\
\text { Sauder School of } \\
\text { Business, } \\
\text { University of } \\
\text { British Columbia }\end{array}$ & $\begin{array}{l}\text { Austria, } \\
\text { The } \\
\text { Netherland } \\
\text { s, Canada }\end{array}$ & $\begin{array}{l}\text { Empirical paper } \\
\text { (quantitative } \\
\text { approach: } \\
\text { multiple } \\
\text { surveys) }\end{array}$ \\
\hline 2012 & Management & $\begin{array}{l}\text { Lee } \quad \text { S.M., } \\
\text { Olson David } \\
\text { L., Trimi S. }\end{array}$ & $\begin{array}{l}\text { Management } \\
\text { Decision }\end{array}$ & 3.787 & $\begin{array}{l}\text { Evolution of value } \\
\text { creation, from closed to } \\
\text { collaborative, open and } \\
\text { co-innovation }\end{array}$ & $\begin{array}{l}\text { co-creation, } \\
\text { co-innovation, } \\
\text { collaboration, } \\
\text { convergenomics, } \\
\text { evolution of } \\
\text { innovation, } \\
\text { innovation, } \\
\text { innovation } \\
\text { imperative, } \\
\text { partnership, value } \\
\text { creation }\end{array}$ & $\begin{array}{l}\text { Co-innovation is a } \\
\text { paradigm where } \\
\text { new ideas and } \\
\text { approaches from } \\
\text { various internal } \\
\text { and external } \\
\text { sources are } \\
\text { integrated in a } \\
\text { platform to } \\
\text { generate new and } \\
\text { shared values } \\
\text { difficult to imitate } \\
\text { by competition. It } \\
\text { includes } \\
\text { engagement and } \\
\text { compelling } \\
\text { experience for } \\
\text { value co-creation } \\
\text { with stakeholders }\end{array}$ & $\begin{array}{l}\text { Department of } \\
\text { Management, } \\
\text { University of } \\
\text { Nebraska }\end{array}$ & USA & $\begin{array}{l}\text { Conceptual/ } \\
\text { theoretical } \\
\text { paper }\end{array}$ \\
\hline 2012 & Management & $\begin{array}{l}\text { Chesbrough } \\
\text { H. }\end{array}$ & $\begin{array}{l}\text { California } \\
\text { Management } \\
\text { Review }\end{array}$ & 1.667 & $\begin{array}{l}\text { Experiment In Open } \\
\text { Innovation }\end{array}$ & not provided & $\begin{array}{l}\text { Process analysis } \\
\text { and description of a } \\
\text { collaborative } \\
\text { temporary platform } \\
\text { to collect ideas } \\
\text { from outside }\end{array}$ & $\begin{array}{l}\text { Haas School of } \\
\text { Business, } \\
\text { University of } \\
\text { California-Berke } \\
\text { ley }\end{array}$ & USA & $\begin{array}{l}\text { Empirical paper } \\
\text { (single case } \\
\text { study) }\end{array}$ \\
\hline 2012 & $\begin{array}{l}\text { Technology } \\
\text { and } \\
\text { Innovation } \\
\text { Management }\end{array}$ & $\begin{array}{l}\text { Dahlander L., } \\
\text { Frederiksen L. }\end{array}$ & $\begin{array}{l}\text { Organization } \\
\text { Science }\end{array}$ & 3.351 & $\begin{array}{l}\text { How relationships in } \\
\text { user communities } \\
\text { provide opportunities to } \\
\text { innovate }\end{array}$ & $\begin{array}{l}\text { collaboration, } \\
\text { communities, } \\
\text { innovation, } \\
\text { network, online, } \\
\text { users }\end{array}$ & $\begin{array}{l}\text { Dispositional } \\
\text { explanations for } \\
\text { user innovation } \\
\text { should be } \\
\text { complemented by a } \\
\text { relational view that } \\
\text { emphasizes how } \\
\text { communities differ }\end{array}$ & $\begin{array}{l}\text { ESMT European } \\
\text { School of } \\
\text { Management and } \\
\text { Technology; } \\
\text { Aarhus } \\
\text { University, } \\
\text { Business and } \\
\text { Social Sciences }\end{array}$ & $\begin{array}{l}\text { Germany, } \\
\text { Denmark }\end{array}$ & $\begin{array}{l}\text { Empirical paper } \\
\text { (mixed } \\
\text { approach: } \\
\text { quantitative- } \\
\text { survey-, } \\
\text { qualitative- } \\
\text { interviews -) }\end{array}$ \\
\hline
\end{tabular}




\begin{tabular}{|c|c|c|c|c|c|c|c|c|c|c|}
\hline & & & & & & & $\begin{array}{l}\text { from other firms, } \\
\text { the types of } \\
\text { behaviors this } \\
\text { enables, and the } \\
\text { effects on } \\
\text { innovation }\end{array}$ & & & \\
\hline 2012 & $\begin{array}{l}\text { Innovation } \\
\text { Management }\end{array}$ & $\begin{array}{l}\text { Mahr D., } \\
\text { Lievens A. }\end{array}$ & Research Policy & 2.850 & $\begin{array}{l}\text { Creation of } \\
\text { related-knowledge } \\
\text { innovation in virtual } \\
\text { communities visited by } \\
\text { lead users }\end{array}$ & $\begin{array}{l}\text { lead users, virtual } \\
\text { communities, } \\
\text { Knowledge value, } \\
\text { innovation }\end{array}$ & $\begin{array}{l}\text { Contributions } \\
\text { given by lead users } \\
\text { in a proactive way } \\
\text { contain more novel } \\
\text { insights than } \\
\text { reactive } \\
\text { contributions such } \\
\text { as answers to } \\
\text { community } \\
\text { members' } \\
\text { questions. The } \\
\text { digital context } \\
\text { favors the creation } \\
\text { of explicit } \\
\text { knowledge that can } \\
\text { be easily integrated } \\
\text { into the } \\
\text { development of } \\
\text { new products }\end{array}$ & $\begin{array}{l}\text { Maastricht } \\
\text { University; } \\
\text { University of } \\
\text { Antwerp }\end{array}$ & $\begin{array}{l}\text { The } \\
\text { Netherland } \\
\text { s, Belgium }\end{array}$ & $\begin{array}{l}\text { Empirical paper } \\
\text { (single case } \\
\text { study- } \\
\text { qualitative } \\
\text { approach: } \\
\text { netnography, } \\
\text { CAT-Consensu } \\
\text { al Agreement } \\
\text { Technique) }\end{array}$ \\
\hline 2012 & $\begin{array}{l}\text { Innovation } \\
\text { Management }\end{array}$ & $\begin{array}{l}\text { Russo-Spena } \\
\text { T., Mele C. }\end{array}$ & $\begin{array}{l}\text { Journal of } \\
\text { Service } \\
\text { Management }\end{array}$ & 1.864 & $\begin{array}{l}\text { Innovation as a process } \\
\text { of co-creation; } \\
\text { innovators as carriers of } \\
\text { practices }\end{array}$ & $\begin{array}{l}\text { "Co-s", } \\
\text { co-creation, } \\
\text { innovating, } \\
\text { innovation, } \\
\text { practices, } \\
\text { value-creating } \\
\text { processes, } \\
\text { working practices }\end{array}$ & $\begin{array}{l}\text { Five "Co-s" model: } \\
\text { co-ideation, } \\
\text { co-valuation, } \\
\text { co-design, co-test, } \\
\text { co-launch. Each } \\
\text { "Co-“ represents a } \\
\text { phase of the } \\
\text { innovation process } \\
\text { resulting from } \\
\text { dynamic and } \\
\text { on-going ing } \\
\text { interactions among } \\
\text { resources, actions, } \\
\text { tools, knowledge, } \\
\text { actors interrelated } \\
\text { and implicates } \\
\text { specific practices. } \\
\text { Managers should } \\
\text { influence } \\
\text { co-creation phases } \\
\text { by contributing to } \\
\text { script practices }\end{array}$ & $\begin{array}{l}\text { Department of } \\
\text { Business } \\
\text { Economics, } \\
\text { University of } \\
\text { Naples "Federico } \\
\text { II" }\end{array}$ & Italy & $\begin{array}{l}\text { Empirical paper } \\
\text { (multiple case } \\
\text { study) }\end{array}$ \\
\hline 2012 & ICT & $\begin{array}{l}\text { Estellés-Arola } \\
\mathrm{s} \quad \mathrm{E} ., \\
\text { González-Lad } \\
\text { rón-de-Gueva } \\
\text { ra F. }\end{array}$ & $\begin{array}{l}\text { Journal of } \\
\text { Information } \\
\text { science }\end{array}$ & 1.238 & $\begin{array}{l}\text { Crowdsourcing } \\
\text { definition }\end{array}$ & $\begin{array}{l}\text { crowdsourcing, } \\
\text { definition, } \\
\text { innovation }\end{array}$ & $\begin{array}{l}\text { Crowdsourcing is a } \\
\text { type of } \\
\text { participative online } \\
\text { activity in which } \\
\text { the firm proposes } \\
\text { to a group of } \\
\text { individuals of } \\
\text { varying knowledge } \\
\text { and number, via a } \\
\text { flexible open call, } \\
\text { the voluntary } \\
\text { undertaking of a } \\
\text { task of variable } \\
\text { complexity. The } \\
\text { user will receive } \\
\text { the satisfaction of a } \\
\text { type of need, be it } \\
\text { economic, social } \\
\text { recognition, } \\
\text { self-esteem, or the }\end{array}$ & $\begin{array}{l}\text { Technical } \\
\text { University of } \\
\text { Valencia }\end{array}$ & Spain & $\begin{array}{l}\text { Conceptual/ } \\
\text { theoretical } \\
\text { paper }\end{array}$ \\
\hline
\end{tabular}




\begin{tabular}{|c|c|c|c|c|c|c|c|c|c|c|}
\hline & & & & & & & $\begin{array}{l}\text { development of } \\
\text { individual skills }\end{array}$ & & & \\
\hline 2012 & $\begin{array}{l}\text { Product } \\
\text { management / } \\
\text { Marketing }\end{array}$ & $\begin{array}{l}\text { Gyrd-Jones } \\
\text { R.I., Kornum } \\
\text { N. }\end{array}$ & $\begin{array}{l}\text { Journal } \\
\text { Business } \\
\text { Research }\end{array}$ & 1.484 & $\begin{array}{l}\text { Co-created brand: } \\
\text { relations with the } \\
\text { stakeholder ecosystems }\end{array}$ & $\begin{array}{l}\text { co-creation, } \\
\text { multi-stakeholder } \\
\text { interaction, online } \\
\text { communities, } \\
\text { complementarity }\end{array}$ & $\begin{array}{l}\text { Successful } \\
\text { co-creation } \\
\text { outcomes are } \\
\text { dependent on: } \\
\text { dialogue and } \\
\text { interaction, value } \\
\text { and cultural } \\
\text { complementarities, } \\
\text { maintenance of } \\
\text { identities of the } \\
\text { parties (lead-users } \\
\text { and firm's staff). If } \\
\text { the firm desires } \\
\text { longevity for a } \\
\text { co-creation project } \\
\text { within a user } \\
\text { community, it } \\
\text { needs not only } \\
\text { listen to the core } \\
\text { lead-users, but also } \\
\text { to the wider } \\
\text { ecosystem (other } \\
\text { committed } \\
\text { consumers) }\end{array}$ & $\begin{array}{l}\text { Griffith } \\
\text { University; } \\
\text { Copenhagen } \\
\text { Business School }\end{array}$ & $\begin{array}{l}\text { Australia, } \\
\text { Denmark }\end{array}$ & $\begin{array}{l}\text { Empirical paper } \\
\text { (single case } \\
\text { study) }\end{array}$ \\
\hline 2011 & $\begin{array}{l}\text { Marketing } \\
\text { management }\end{array}$ & $\begin{array}{l}\text { Cova B., Dalli } \\
\text { D., Zwick D. }\end{array}$ & $\begin{array}{l}\text { Marketing } \\
\text { Theory }\end{array}$ & 0.953 & $\begin{array}{lll}\text { Perspectives } & & \text { on } \\
\text { consumers' role } & \text { as } \\
\text { 'producers' } & & \end{array}$ & $\begin{array}{l}\text { collaborative } \\
\text { capitalism, } \\
\text { consumer work, } \\
\text { exploitation, } \\
\text { prosumption, } \\
\text { service-dominant } \\
\text { logic, value } \\
\text { co-creation }\end{array}$ & $\begin{array}{l}\text { Understanding of } \\
\text { the place of } \\
\text { marketing } \\
\text { techniques in value } \\
\text { creation }\end{array}$ & $\begin{array}{l}\text { Euromed } \\
\text { Management } \\
\text { Marseille; } \\
\text { University of } \\
\text { Pisa; Schulich } \\
\text { School of } \\
\text { Business York } \\
\text { University } \\
\end{array}$ & $\begin{array}{l}\text { France, } \\
\text { Italy, } \\
\text { Canada }\end{array}$ & $\begin{array}{l}\text { Conceptual/ } \\
\text { theoretical } \\
\text { paper }\end{array}$ \\
\hline 2011 & Marketing & $\begin{array}{l}\text { Frow P., } \\
\text { Payne A.F., } \\
\text { Storbacka K. }\end{array}$ & $\begin{array}{l}\text { Proceedings of } \\
\text { ANZMAC } \\
\text { Conference }\end{array}$ & - & Concept of co-creation & $\begin{array}{l}\text { co-creation, } \\
\text { co-production, } \\
\text { taxonomy, } \\
\text { conceptual } \\
\text { framework }\end{array}$ & $\begin{array}{l}\text { Identification of } \\
\text { key forms of } \\
\text { co-creation }\end{array}$ & $\begin{array}{lr}\text { University } & \text { of } \\
\text { Sidney; } & \\
\text { University } & \text { of } \\
\text { New South } & \text { Wales; Hanken } \\
\text { School } & \text { of } \\
\text { Economics } & \\
\end{array}$ & $\begin{array}{l}\text { Australia, } \\
\text { Finland }\end{array}$ & $\begin{array}{l}\text { Conceptual/ } \\
\text { theoretical } \\
\text { paper }\end{array}$ \\
\hline 2011 & $\begin{array}{l}\text { Production } \\
\text { Management }\end{array}$ & $\begin{array}{l}\text { Romero D., } \\
\text { Molina A. }\end{array}$ & $\begin{array}{l}\text { Production } \\
\text { Planning \& } \\
\text { Control: The } \\
\text { Management of } \\
\text { Operations }\end{array}$ & - & $\begin{array}{l}\text { Collaborative networked } \\
\text { firms and VCCs }\end{array}$ & $\begin{array}{l}\text { CNOs; customer } \\
\text { communities; } \\
\text { interactive } \\
\text { marketing; } \\
\text { co-innovation; OI; } \\
\text { value co-creation; } \\
\text { value systems; } \\
\text { virtual breeding } \\
\text { environments; } \\
\text { virtual } \\
\text { organizations }\end{array}$ & $\begin{array}{l}\text { Framework for } \\
\text { creating interface } \\
\text { networks in order } \\
\text { to support } \\
\text { user-driven } \\
\text { innovation }\end{array}$ & $\begin{array}{l}\text { Tecnologico de } \\
\text { Monterrey }\end{array}$ & Mexico & $\begin{array}{l}\text { Conceptual/ } \\
\text { theoretical } \\
\text { paper }\end{array}$ \\
\hline 2011 & Marketing & $\begin{array}{l}\text { Berthon, P.R., } \\
\text { Campbell } \\
\text { C.L., Pitt L.F., } \\
\text { McCarthy I. }\end{array}$ & $\begin{array}{l}\text { Journal of } \\
\text { Consumer } \\
\text { Marketing }\end{array}$ & - & $\begin{array}{l}\text { Creative consumers and } \\
\text { firm's stance towards } \\
\text { them }\end{array}$ & $\begin{array}{l}\text { consumers, } \\
\text { creative } \\
\text { consumers, } \\
\text { creative thinking, } \\
\text { firm response, } \\
\text { instruments, } \\
\text { modifying }\end{array}$ & $\begin{array}{l}\text { Construction of a } \\
\text { scale for measuring } \\
\text { a firm's stance } \\
\text { toward creative } \\
\text { consumers }\end{array}$ & $\begin{array}{l}\text { Monash } \\
\text { University; } \\
\text { Bentley } \\
\text { University; } \\
\text { Simon Fraser } \\
\text { University }\end{array}$ & $\begin{array}{l}\text { Australia, } \\
\text { USA, } \\
\text { Canada }\end{array}$ & $\begin{array}{l}\text { Methodological } \\
\text { paper }\end{array}$ \\
\hline 2011 & $\begin{array}{l}\text { Technology } \\
\text { and } \\
\text { Information } \\
\text { Management }\end{array}$ & $\begin{array}{lr}\text { Kohler } & \text { T., } \\
\text { Füller } & \text { J., } \\
\text { Matzler } & \text { K., } \\
\text { Stieger D. } & \end{array}$ & MIS Quarterly & 4.659 & $\begin{array}{l}\text { Emerging virtual worlds } \\
\text { and opportunities for } \\
\text { firms to collaborate with } \\
\text { co-creating users }\end{array}$ & $\begin{array}{l}\text { virtual worlds, } \\
\text { action research, } \\
\text { co-creation, } \\
\text { experience design, } \\
\text { SecondLife }\end{array}$ & $\begin{array}{l}\text { Conception and } \\
\text { testing of a virtual } \\
\text { co-creation system } \\
\text { called Ideation } \\
\text { Quest, revealing } \\
\text { how to design } \\
\text { co-creation } \\
\text { systems in the }\end{array}$ & $\begin{array}{l}\text { College of BA, } \\
\text { Hawaii Pacific } \\
\text { University; } \\
\text { Innsbruck } \\
\text { University } \\
\text { School of } \\
\text { Management }\end{array}$ & $\begin{array}{l}\text { USA, } \\
\text { Austria }\end{array}$ & $\begin{array}{l}\text { Methodological } \\
\text { paper }\end{array}$ \\
\hline
\end{tabular}




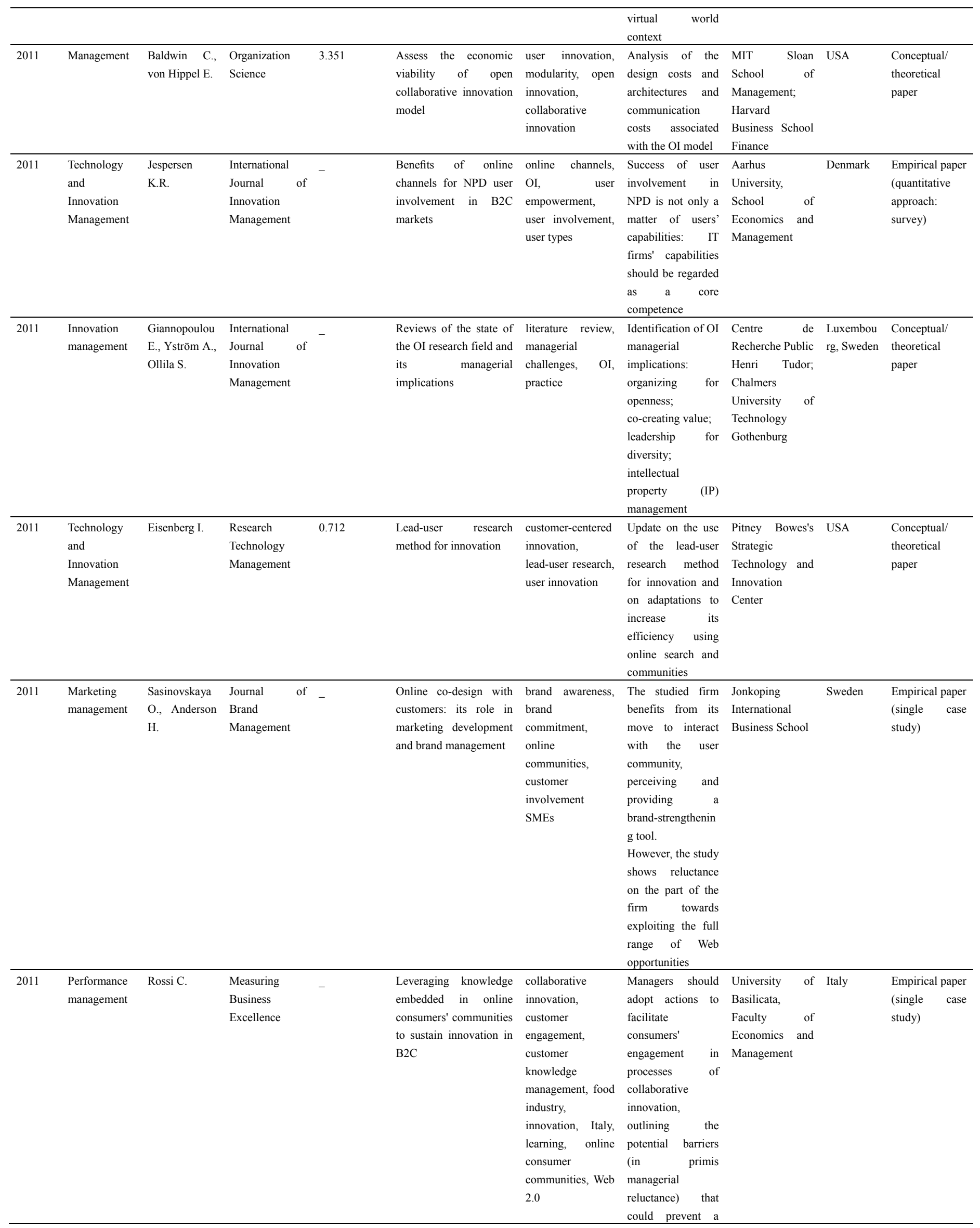




\begin{tabular}{|c|c|c|c|c|c|c|c|c|c|c|}
\hline & & & & & & & $\begin{array}{l}\text { successful } \\
\text { co-creation } \\
\text { outcome: } \\
\text { combination of } \\
\text { strategies } \\
\text { addressed at } \\
\text { accruing not only } \\
\text { users' motivation } \\
\text { but also managerial } \\
\text { commitment }\end{array}$ & & & \\
\hline 2011 & $\begin{array}{l}\text { Innovation } \\
\text { Management / } \\
\text { NPD }\end{array}$ & $\begin{array}{l}\text { Fuchs C., } \\
\text { Schreier M. }\end{array}$ & $\begin{array}{l}\text { Journal of } \\
\text { Product } \\
\text { Innovation } \\
\text { Management }\end{array}$ & 1.572 & $\begin{array}{l}\text { Customer empowerment } \\
\text { in NPD: how customers } \\
\text { from the "periphery" } \\
\text { perceive empowerment } \\
\text { strategies }\end{array}$ & not provided & 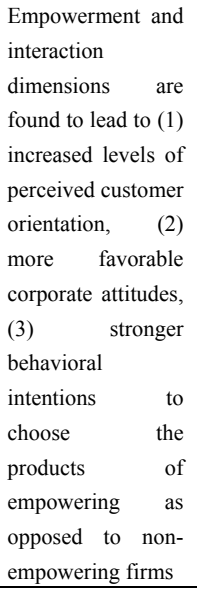 & $\begin{array}{l}\text { Rotterdam } \\
\text { School of } \\
\text { Management; } \\
\text { Bocconi } \\
\text { University }\end{array}$ & $\begin{array}{l}\text { The } \\
\text { Netherland } \\
\text { s, Italy }\end{array}$ & $\begin{array}{l}\text { Empirical paper } \\
\text { (quantitative } \\
\text { approach- } \\
\text { multiple } \\
\text { surveys-) }\end{array}$ \\
\hline 2011 & Management & $\begin{array}{l}\text { Lichtenthaler } \\
\text { U. }\end{array}$ & $\begin{array}{l}\text { The Academy } \\
\text { of Management } \\
\text { Perspectives }\end{array}$ & 3.174 & $\begin{array}{l}\text { Open Innovation: state } \\
\text { of the art }\end{array}$ & not provided & $\begin{array}{l}\text { Definition of OI } \\
\text { delimiting it from } \\
\text { open source; } \\
\text { overview of prior } \\
\text { research, which } \\
\text { identifies key } \\
\text { topics of earlier } \\
\text { research: } \\
\text { technology } \\
\text { transactions, user } \\
\text { innovation, } \\
\text { business models, } \\
\text { innovation } \\
\text { markets. }\end{array}$ & $\begin{array}{l}\text { University of } \\
\text { Mannheim }\end{array}$ & Germany & $\begin{array}{l}\text { Conceptual/ } \\
\text { theoretical } \\
\text { paper }\end{array}$ \\
\hline 2010 & NPD & $\begin{array}{l}\text { Hoyer W., } \\
\text { Chandy R., } \\
\text { Dorotic M., } \\
\text { Krafft M., } \\
\text { Singh S.S. }\end{array}$ & $\begin{array}{l}\text { Journal } \\
\text { Service } \\
\text { Research }\end{array}$ & 2.714 & $\begin{array}{l}\text { Consumer cocreation in } \\
\text { NPD }\end{array}$ & $\begin{array}{l}\text { cocreation, } \\
\text { conceptual , } \\
\text { theoretical, } \\
\text { innovations, NPI, } \\
\text { value }\end{array}$ & $\begin{array}{l}\text { Impact of } \\
\text { co-creation at each } \\
\text { stage of the NPD } \\
\text { process. } \\
\text { Identification of } \\
\text { areas for future } \\
\text { research }\end{array}$ & $\begin{array}{l}\text { McCombs } \\
\text { School of } \\
\text { Business, } \\
\text { University of } \\
\text { Texas at Austin; } \\
\text { London Business } \\
\text { School; } \\
\text { University of } \\
\text { Groningen; } \\
\text { University of } \\
\text { Muenster; } \\
\text { Rice University }\end{array}$ & $\begin{array}{l}\text { USA, UK, } \\
\text { The } \\
\text { Netherland } \\
\text { s, } \\
\text { Germany }\end{array}$ & $\begin{array}{l}\text { Conceptual/ } \\
\text { theoretical } \\
\text { paper }\end{array}$ \\
\hline 2010 & $\begin{array}{l}\text { Product } \\
\text { management / } \\
\text { Marketing }\end{array}$ & $\begin{array}{l}\text { Hatch MJ., } \\
\text { Schultz M. }\end{array}$ & $\begin{array}{l}\text { Journal of } \\
\text { Brand } \\
\text { Management }\end{array}$ & - & Brand co-creation & $\begin{array}{l}\text { brand governance, } \\
\text { brands and } \\
\text { branding, } \\
\text { co-creation, } \\
\text { company } \\
\text { stakeholder } \\
\text { engagement, } \\
\text { organizational } \\
\text { self-disclosure }\end{array}$ & $\begin{array}{l}\text { Link between } \\
\text { co-creation and } \\
\text { brand governance; } \\
\text { marketing-based } \\
\text { model reframing of } \\
\text { co-creation from a } \\
\text { longitudinal case } \\
\text { study }\end{array}$ & $\begin{array}{l}\text { University of } \\
\text { Virginia; } \\
\text { Copenhagen } \\
\text { Business School }\end{array}$ & $\begin{array}{l}\text { USA, } \\
\text { Denmark }\end{array}$ & $\begin{array}{l}\text { Empirical paper } \\
\text { (single case } \\
\text { study) }\end{array}$ \\
\hline 2010 & $\begin{array}{l}\text { Innovation } \\
\text { Management }\end{array}$ & $\begin{array}{l}\text { Morgan } \quad \text { J., } \\
\text { Wang R }\end{array}$ & $\begin{array}{l}\text { California } \\
\text { Management } \\
\text { Review }\end{array}$ & 1.667 & $\begin{array}{l}\text { Contests designed to } \\
\text { produce innovation }\end{array}$ & not provided & $\begin{array}{l}\text { Decision tree } \\
\text { which includes the } \\
\text { major points in the } \\
\text { design of } \\
\text { tournaments for }\end{array}$ & $\begin{array}{l}\text { Haas School of } \\
\text { Business, UC } \\
\text { Berkeley }\end{array}$ & USA & $\begin{array}{l}\text { Conceptual/ } \\
\text { theoretical } \\
\text { paper }\end{array}$ \\
\hline
\end{tabular}




\begin{tabular}{|c|c|c|c|c|c|c|c|c|c|c|}
\hline & & & & & & & ideas is discussed & & & \\
\hline 2010 & Management & $\begin{array}{l}\text { Nambisan S., } \\
\text { Baron R.A. }\end{array}$ & $\begin{array}{l}\text { Organization } \\
\text { Science }\end{array}$ & 3.351 & $\begin{array}{l}\text { Organizing } \begin{array}{l}\text { VCEs to } \\
\text { promote }\end{array} \\
\text { contributions }\end{array}$ & $\begin{array}{l}\text { VCE, customer } \\
\text { co-innovation, } \\
\text { product support, } \\
\text { customer } \\
\text { identification, } \\
\text { CRM }\end{array}$ & 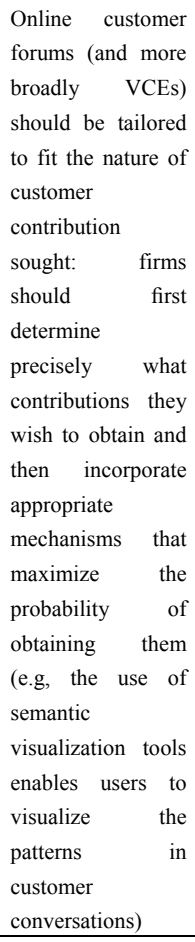 & $\begin{array}{l}\text { Lally School of } \\
\text { Management, } \\
\text { Rensselaer } \\
\text { Polytechnic } \\
\text { Institute; Spears } \\
\text { School } \\
\text { Business, } \\
\text { Oklahoma State } \\
\text { University }\end{array}$ & USA & $\begin{array}{l}\text { Empirical paper } \\
\text { (quantitative } \\
\text { approach- } \\
\text { online } \\
\text { surveys-) }\end{array}$ \\
\hline 2010 & $\begin{array}{l}\text { Business } \\
\text { research }\end{array}$ & Zwass, V. & $\begin{array}{l}\text { International } \\
\text { Journal } \\
\text { Electronic } \\
\text { Commerce }\end{array}$ & of 1.425 & Concept of co-creation & $\begin{array}{l}\text { active } \\
\text { consumption, } \\
\text { co-creation, } \\
\text { consumer roles, } \\
\text { e-commerce } \\
\text { research, } \\
\text { taxonomic } \\
\text { frameworks }\end{array}$ & $\begin{array}{l}\text { Typology of } \\
\text { co-created value } \\
\text { and taxonomy } \\
\text { framework of } \\
\text { factors } \\
\text { Web-based of } \\
\text { co-creation in } \\
\text { autonomously and } \\
\text { in symbiosis with } \\
\text { producer } \\
\text { organizations }\end{array}$ & $\begin{array}{l}\text { Fairleigh } \\
\text { Dickinson } \\
\text { University }\end{array}$ & USA & $\begin{array}{l}\text { Conceptual/ } \\
\text { theoretical } \\
\text { paper }\end{array}$ \\
\hline 2010 & $\begin{array}{l}\text { Technology } \\
\text { and } \\
\text { Innovation } \\
\text { Management }\end{array}$ & $\begin{array}{l}\text { Wu S.C., Fang } \\
\text { W.C. }\end{array}$ & Technovation & 3.177 & $\begin{array}{l}\mathrm{C} 2 \mathrm{C} \text { interactions and } \\
\text { idea generation in virtual } \\
\text { brand communities }\end{array}$ & $\begin{array}{l}\text { consumer-to-cons } \\
\text { umer interaction, } \\
\text { human capital, } \\
\text { brand knowledge, } \\
\text { idea generation } \\
\end{array}$ & $\begin{array}{l}\mathrm{C} 2 \mathrm{C} \text { interactions } \\
\text { are positively } \\
\text { associated with } \\
\text { idea generation }\end{array}$ & $\begin{array}{l}\text { National Taipei } \\
\text { University; } \\
\text { Chihlee Institute } \\
\text { of Technology }\end{array}$ & $\begin{array}{l}\text { Taiwan } \\
\text { (Republic } \\
\text { of China) }\end{array}$ & $\begin{array}{l}\text { Empirical paper } \\
\text { (single case } \\
\text { study) }\end{array}$ \\
\hline 2010 & $\begin{array}{l}\text { Innovation } \\
\text { Management / } \\
\text { NPD }\end{array}$ & $\begin{array}{l}\text { Jespersen, } \\
\text { K.R. }\end{array}$ & $\begin{array}{l}\text { International } \\
\text { Journal } \\
\text { Innovation } \\
\text { Management }\end{array}$ & of & $\begin{array}{l}\text { User-involvement in OI } \\
\text { and decision-maker } \\
\text { openness }\end{array}$ & $\begin{array}{l}\text { cognitive distance, } \\
\text { decision-maker } \\
\text { openness, } \\
\text { involvement } \\
\text { intensity, NPD } \\
\text { stages, OI, user } \\
\text { types, } \\
\text { user-involvement }\end{array}$ & $\begin{array}{l}\text { The user } \\
\text { involvement } \\
\text { process is } \\
\text { moderated by the } \\
\text { decision-makers } \\
\text { openness }\end{array}$ & $\begin{array}{l}\text { Aarhus } \\
\text { University, } \\
\text { School of } \\
\text { Economics and } \\
\text { Management }\end{array}$ & Denmark & $\begin{array}{l}\text { Empirical paper } \\
\text { (quantitative } \\
\text { approach: } \\
\text { survey) }\end{array}$ \\
\hline 2010 & $\begin{array}{l}\text { Technology } \\
\text { and } \\
\text { Innovation } \\
\text { Management }\end{array}$ & $\begin{array}{l}\text { Heiskanen E., } \\
\text { Hyysalo S., } \\
\text { Kotro T., Repo } \\
\text { P. }\end{array}$ & $\begin{array}{l}\text { Technology } \\
\text { Analysis } \\
\text { Strategic } \\
\text { Management }\end{array}$ & $\begin{array}{ll} & 1.095 \\
\& & \end{array}$ & $\begin{array}{l}\text { User-inclusive } \\
\text { innovation communitites }\end{array}$ & $\begin{array}{l}\text { user, innovation, } \\
\text { innovation } \\
\text { community, } \\
\text { community of } \\
\text { practice }\end{array}$ & $\begin{array}{l}\text { An innovation } \\
\text { community needs } \\
\text { to include at least } \\
\text { one community of } \\
\text { practice; firms } \\
\text { should consider } \\
\text { their actions in } \\
\text { fostering } \\
\text { interaction and in } \\
\text { responding to } \\
\text { users' initiatives }\end{array}$ & $\begin{array}{l}\text { University } \quad \text { of } \\
\text { Helsinki; NCR } \\
\text { Centre Helsinki }\end{array}$ & Finland & $\begin{array}{l}\text { Empirical paper } \\
\text { (multiple case } \\
\text { study) }\end{array}$ \\
\hline 2010 & Management & Füller J. & $\begin{array}{l}\text { California } \\
\text { Management } \\
\text { Review }\end{array}$ & 1.667 & $\begin{array}{lr}\text { Virtual } & \text { co-creation } \\
\text { refined from a } & \text { social } \\
\text { exchange } & \text { theory } \\
\text { perspective } & \end{array}$ & not provided & $\begin{array}{l}\text { Consumers } \\
\text { co-creation beliefs } \\
\text { differ along various } \\
\text { aspects such as the }\end{array}$ & $\begin{array}{l}\text { Innsbruck } \\
\text { University } \\
\text { School of } \\
\text { Management }\end{array}$ & Austria & $\begin{array}{l}\text { Empirical paper } \\
\text { (quantitative } \\
\text { approach: } \\
\text { survey) }\end{array}$ \\
\hline
\end{tabular}




\begin{tabular}{|c|c|c|c|c|c|c|c|c|c|c|}
\hline & & & & & & & $\begin{array}{l}\text { preferred } \\
\text { interaction partner, } \\
\text { the intensity and } \\
\text { extent of } \\
\text { participation }\end{array}$ & & & \\
\hline 2010 & $\begin{array}{l}\text { Innovation } \\
\text { Management }\end{array}$ & $\begin{array}{l}\text { Gianiodis P.T., } \\
\text { Ellis S.C., } \\
\text { Secchi E. }\end{array}$ & $\begin{array}{l}\text { International } \\
\text { Journal of } \\
\text { Innovation } \\
\text { Management }\end{array}$ & _ & $\begin{array}{l}\text { Conceptual framework } \\
\text { of OI }\end{array}$ & $\begin{array}{l}\text { innovation broker, } \\
\text { innovation } \\
\text { sourcing, OI, } \\
\text { technological } \\
\text { change, typology }\end{array}$ & 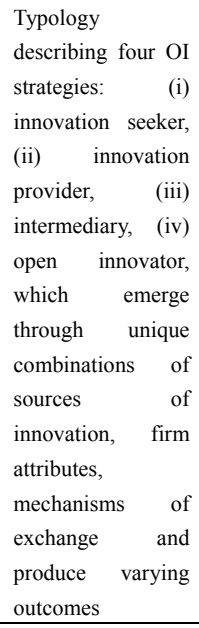 & $\begin{array}{l}\text { College of } \\
\text { Business and } \\
\text { Behavioral } \\
\text { Science, } \\
\text { Clemson } \\
\text { University; } \\
\text { Gatton College } \\
\text { of Business \& } \\
\text { Economics, } \\
\text { University of } \\
\text { Kentucky }\end{array}$ & USA & $\begin{array}{l}\text { Conceptual/ } \\
\text { theoretical } \\
\text { paper }\end{array}$ \\
\hline 2010 & $\begin{array}{l}\text { Technology } \\
\text { and Society }\end{array}$ & Brabham D.C. & $\begin{array}{l}\text { Information, } \\
\text { Communication } \\
\& \text { Society }\end{array}$ & 0.700 & Crowdsourcing & $\begin{array}{l}\text { addiction, } \\
\text { crowdsourcing, } \\
\text { instant messaging } \\
\text { interviews, } \\
\text { motivations, } \\
\text { online community }\end{array}$ & $\begin{array}{l}\text { Four primary } \\
\text { motivators for } \\
\text { participation at } \\
\text { Threadless (case } \\
\text { study) have } \\
\text { emerged: the } \\
\text { opportunity to } \\
\text { make money, the } \\
\text { opportunity to } \\
\text { develop one's } \\
\text { creative skills, the } \\
\text { potential to take up } \\
\text { freelance work, the } \\
\text { love of community } \\
\text { at Threadless }\end{array}$ & $\begin{array}{l}\text { School of } \\
\text { Journalism \& } \\
\text { Mass } \\
\text { Communication, } \\
\text { University of } \\
\text { North Carolina } \\
\text { (UNC-Chapel } \\
\text { Hill) }\end{array}$ & USA & $\begin{array}{l}\text { Empirical paper } \\
\text { (qualitative } \\
\text { approach: } \\
\text { interviews) }\end{array}$ \\
\hline 2010 & Management & $\begin{array}{l}\text { Awa H.O., } \\
\text { Eze S.C. }\end{array}$ & $\begin{array}{l}\text { International } \\
\text { Business and } \\
\text { Management }\end{array}$ & - & $\begin{array}{l}\text { User community } \\
\text { collaboration in } \\
\text { innovation }\end{array}$ & $\begin{array}{l}\text { democracy, value } \\
\text { co-creation, user } \\
\text { community, } \\
\text { innovation, } \\
\text { extended } \\
\text { enterprise and } \\
\text { technology }\end{array}$ & $\begin{array}{l}\text { Framework of user } \\
\text { community } \\
\text { collaboration, } \\
\text { suggesting } \\
\text { effective } \\
\text { mobilization of } \\
\text { consumers and } \\
\text { flattened } \\
\text { organizational } \\
\text { structures. } \\
\text { Co-creationist } \\
\text { capitalism is an } \\
\text { instrument to build } \\
\text { competitive } \\
\text { advantage in an } \\
\text { environment where } \\
\text { market power } \\
\text { resides in } \\
\text { immaterial and } \\
\text { symbolic resources }\end{array}$ & $\begin{array}{l}\text { University of } \\
\text { Port Harcourt; } \\
\text { Business and } \\
\text { Management } \\
\text { Research } \\
\text { Institutes, } \\
\text { University of } \\
\text { Bedfordshire }\end{array}$ & $\begin{array}{l}\text { Nigeria, } \\
\text { UK }\end{array}$ & $\begin{array}{l}\text { Conceptual/ } \\
\text { theoretical } \\
\text { paper }\end{array}$ \\
\hline 2010 & $\begin{array}{l}\text { Marketing } \\
\text { management }\end{array}$ & $\begin{array}{l}\text { Harwood } \mathrm{T} ., \\
\text { Garry T. }\end{array}$ & $\begin{array}{l}\text { Journal of } \\
\text { Marketing } \\
\text { Management }\end{array}$ & - & $\begin{array}{l}\text { Virtual co-creation } \\
\text { environments: } \\
\text { post-product } \\
\text { manipulations by } \\
\text { consumers }\end{array}$ & $\begin{array}{l}\text { value co-creation, } \\
\text { participation } \\
\text { marketing, } \\
\text { consumption } \\
\text { community }\end{array}$ & $\begin{array}{l}\text { Findings suggest } \\
\text { consumers are able } \\
\text { to take ownership, } \\
\text { define and create } \\
\text { their own } \\
\text { post-product } \\
\text { consumption } \\
\text { experience, and, } \\
\text { through a }\end{array}$ & $\begin{array}{l}\text { De Montfort } \\
\text { University; } \\
\text { University of } \\
\text { Canterbury }\end{array}$ & $\begin{array}{l}\text { UK, New } \\
\text { Zealand }\end{array}$ & $\begin{array}{l}\text { Empirical paper } \\
\text { (single case } \\
\text { study) }\end{array}$ \\
\hline
\end{tabular}




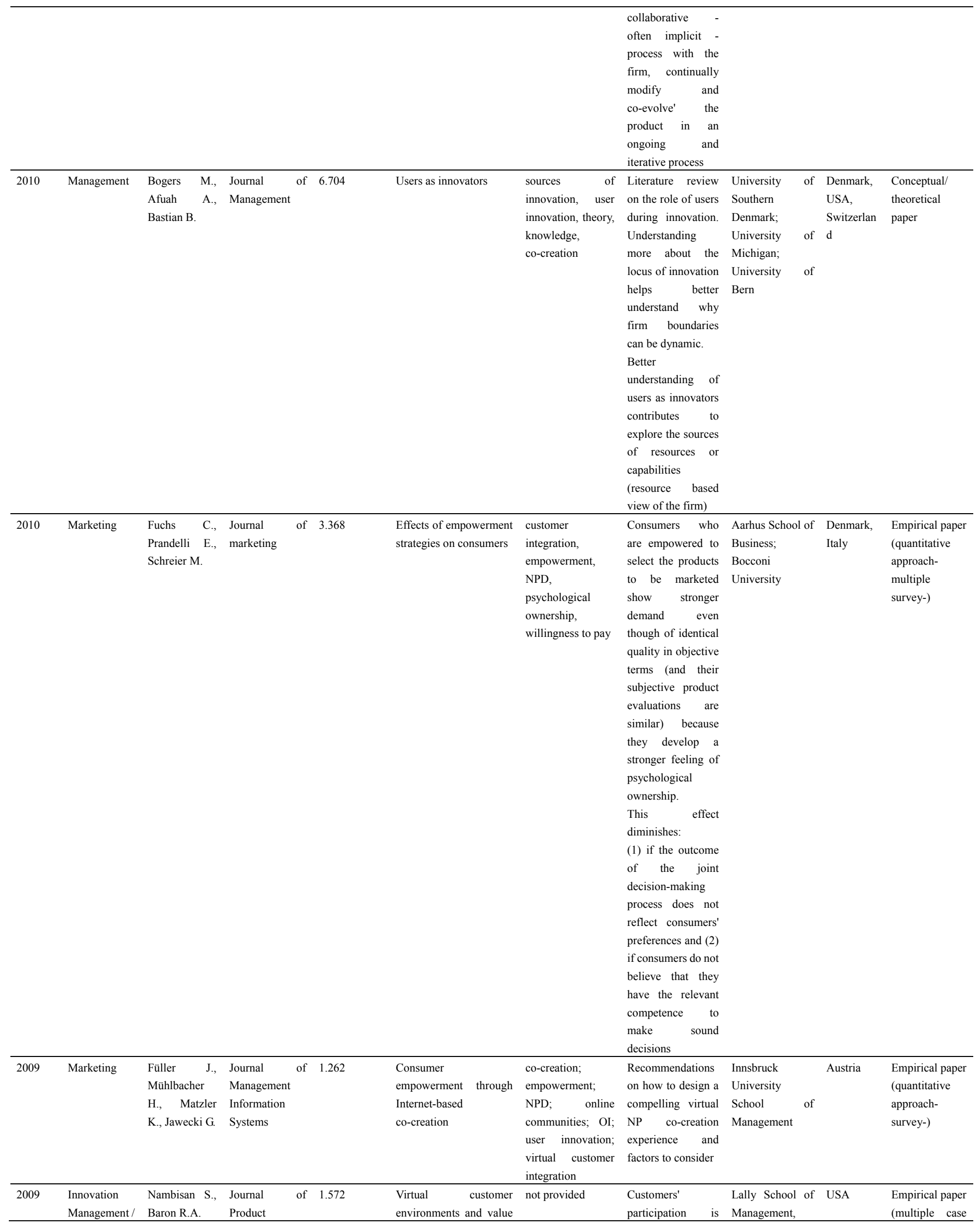




\begin{tabular}{|c|c|c|c|c|c|c|c|c|c|c|}
\hline & NPD & & $\begin{array}{l}\text { Innovation } \\
\text { Management }\end{array}$ & & co-creation activities & & $\begin{array}{l}\text { motivated not just } \\
\text { by their } \\
\text { norm-related } \\
\text { behavior but by the } \\
\text { benefits of } \\
\text { engaging in such } \\
\text { activities }\end{array}$ & $\begin{array}{l}\text { Rensselaer } \\
\text { Polytechnic } \\
\text { Institute, NY; } \\
\text { Spears School of } \\
\text { Business, } \\
\text { Oklahoma State } \\
\text { University } \\
\end{array}$ & & study) \\
\hline 2009 & $\begin{array}{l}\text { Technology } \\
\text { and } \\
\text { Innovation } \\
\text { Management }\end{array}$ & $\begin{array}{l}\text { Ojanen } \quad \text { V., } \\
\text { Hallikas J. }\end{array}$ & $\begin{array}{l}\text { International } \\
\text { Journal of } \\
\text { Technology } \\
\text { Management }\end{array}$ & 0.564 & $\begin{array}{l}\text { Transformation of } \\
\text { customer relationships in } \\
\text { collaborative innovation }\end{array}$ & $\begin{array}{l}\text { collaborative } \\
\text { innovation } \\
\text { learning; customer } \\
\text { relationships; } \\
\text { engineering } \\
\text { industry; } \\
\text { innovation } \\
\text { management; } \\
\text { inter-organizationa } \\
\text { 1 learning / } \\
\text { relationships; } \\
\text { knowledge } \\
\text { intensity; } \\
\text { organizational } \\
\text { learning; routines; } \\
\text { transformation } \\
\text { process }\end{array}$ & $\begin{array}{l}\text { Driving forces and } \\
\text { influence of } \\
\text { inter-organizationa } \\
1 \text { routines in the } \\
\text { transformation } \\
\text { process towards } \\
\text { customer } \\
\text { collaboration in } \\
\text { innovation. As } \\
\text { routines are } \\
\text { generally regarded } \\
\text { as key elements of } \\
\text { organizational } \\
\text { structure and are } \\
\text { seen as the primary } \\
\text { means by which } \\
\text { firms accomplish } \\
\text { much of what they } \\
\text { do, they represent a } \\
\text { very important } \\
\text { aspect of } \\
\text { collaboration }\end{array}$ & $\begin{array}{l}\text { Lappeenranta } \\
\text { University of } \\
\text { Technology }\end{array}$ & Finland & $\begin{array}{l}\text { Empirical paper } \\
\text { (single case } \\
\text { study) }\end{array}$ \\
\hline 2009 & $\begin{array}{l}\text { Business } \\
\text { research }\end{array}$ & Vargo S.L. & $\begin{array}{ll}\text { Journal } & \text { of } \\
\text { Business } \quad \& \\
\text { Industry }\end{array}$ & 1.000 & $\begin{array}{l}\text { Service-dominant-logic- } \\
\text { based conceptualization } \\
\text { of relationship }\end{array}$ & $\begin{array}{l}\text { relationship } \\
\text { marketing, } \\
\text { buyer-seller } \\
\text { relationships, } \\
\text { ecology, } \\
\text { networking }\end{array}$ & $\begin{array}{l}\text { Better } \\
\text { understanding of } \\
\text { the role of } \\
\text { relationship in } \\
\text { value creation and } \\
\text { its correspondence } \\
\text { to transactions and } \\
\text { products }\end{array}$ & $\begin{array}{l}\text { Shidler College } \\
\text { of Business, } \\
\text { University of } \\
\text { Hawaii at Manoa }\end{array}$ & USA & $\begin{array}{l}\text { Conceptual/ } \\
\text { theoretical } \\
\text { paper }\end{array}$ \\
\hline 2009 & $\begin{array}{l}\text { Technology } \\
\text { and } \\
\text { Innovation } \\
\text { Management }\end{array}$ & $\begin{array}{l}\text { Kohler } \quad \text { T., } \\
\text { Matzler } \quad \text { K., } \\
\text { Johann } \\
\text { J. Füller }\end{array}$ & Technovation & 3.177 & $\begin{array}{lr}\text { Opportunities virtual } \\
\text { worlds offer for } \\
\text { innovations }\end{array}$ & $\begin{array}{l}\text { innovation, Avatar, } \\
\text { SecondLife, } \\
\text { co-creation }\end{array}$ & $\begin{array}{l}\text { To realize the } \\
\text { potential of } \\
\text { Avatar-based } \\
\text { innovation, firms } \\
\text { need to create a } \\
\text { compelling OI } \\
\text { experience and } \\
\text { consider the } \\
\text { peculiarities of } \\
\text { virtual worlds }\end{array}$ & $\begin{array}{l}\text { Innsbruck } \\
\text { University } \\
\text { School of } \\
\text { Management }\end{array}$ & Austria & $\begin{array}{l}\text { Empirical paper } \\
\text { (multiple case } \\
\text { study) }\end{array}$ \\
\hline 2008 & $\begin{array}{l}\text { Innovation } \\
\text { Management / } \\
\text { NPD }\end{array}$ & $\begin{array}{l}\text { Bilgram } \\
\text { Brem } \\
\text { Voigt K.-I. }\end{array}$ & $\begin{array}{l}\text { International } \\
\text { Journal of } \\
\text { Innovation } \\
\text { Management }\end{array}$ & - & $\begin{array}{l}\text { User-Centric } \\
\text { Innovations in NPD }\end{array}$ & $\begin{array}{l}\text { NPD, lead user, } \\
\text { Web 2.0, online } \\
\text { communities, } \\
\text { user-centric } \\
\text { innovation }\end{array}$ & $\begin{array}{l}\text { Crucial factors for } \\
\text { the online } \\
\text { identification of } \\
\text { lead users in the } \\
\text { virtual } \\
\text { environment }\end{array}$ & $\begin{array}{l}\text { University of } \\
\text { Erlangen-Nurem } \\
\text { berg }\end{array}$ & Germany & $\begin{array}{l}\text { Conceptual/ } \\
\text { theoretical } \\
\text { paper }\end{array}$ \\
\hline 2008 & Marketing & Etgar M. & $\begin{array}{l}\text { Journal of the } \\
\text { Academy of } \\
\text { Marketing } \\
\text { Science }\end{array}$ & 2.570 & Co-production process & $\begin{array}{l}\text { co-production, } \\
\text { co-creation, } \\
\text { customization, risk } \\
\text { reduction, activity } \\
\text { chains }\end{array}$ & $\begin{array}{l}\text { Descriptive model } \\
\text { of consumer } \\
\text { engagement in } \\
\text { co-production }\end{array}$ & $\begin{array}{l}\text { Graduate School } \\
\text { of BA, College } \\
\text { of Management }\end{array}$ & Israel & $\begin{array}{l}\text { Conceptual/ } \\
\text { theoretical } \\
\text { paper }\end{array}$ \\
\hline 2008 & $\begin{array}{l}\text { Innovation } \\
\text { Management / } \\
\text { NPD }\end{array}$ & $\begin{array}{l}\text { Kim J.H., Bae } \\
\text { Z., Kang S.H. }\end{array}$ & $\begin{array}{l}\text { International } \\
\text { Journal of } \\
\text { Innovation } \\
\text { Management } \\
\end{array}$ & - & $\begin{array}{l}\text { Online brand } \\
\text { communities and NPD }\end{array}$ & $\begin{array}{l}\text { NPD, online } \\
\text { community, role of } \\
\text { users, user } \\
\text { innovation } \\
\end{array}$ & $\begin{array}{l}\text { The roles of online } \\
\text { brand communities } \\
\text { vary along the } \\
\text { NPD stages }\end{array}$ & $\begin{array}{l}\text { KAIST Graduate } \\
\text { School of } \\
\text { Management }\end{array}$ & Korea & $\begin{array}{l}\text { Empirical paper } \\
\text { (multiple case } \\
\text { study) }\end{array}$ \\
\hline 2008 & $\begin{array}{l}\text { Innovation } \\
\text { Management }\end{array}$ & $\begin{array}{l}\text { Dahlander L., } \\
\text { Frederiksen } \\
\text { L., Rullani F. }\end{array}$ & $\begin{array}{l}\text { Industry and } \\
\text { Innovation }\end{array}$ & 0.790 & $\begin{array}{l}\text { Online communities and } \\
\text { OI }\end{array}$ & not provided & $\begin{array}{l}\text { Highlighting the } \\
\text { role of individual } \\
\text { users embedded in } \\
\text { online } \\
\text { communities for }\end{array}$ & $\begin{array}{l}\text { Imperial College } \\
\text { London; } \\
\text { Copenhagen } \\
\text { Business Scool }\end{array}$ & $\begin{array}{l}\text { UK, } \\
\text { Denmark }\end{array}$ & $\begin{array}{l}\text { Conceptual/ } \\
\text { theoretical } \\
\text { paper }\end{array}$ \\
\hline
\end{tabular}




\begin{tabular}{|c|c|c|c|c|c|c|c|c|c|c|}
\hline & & & & & & & $\begin{array}{l}\text { distributed and } \\
\text { cumulative } \\
\text { innovation }\end{array}$ & & & \\
\hline 2008 & $\begin{array}{l}\text { Innovation } \\
\text { Management }\end{array}$ & $\begin{array}{l}\text { West J., } \\
\text { Lakhani K.R. }\end{array}$ & $\begin{array}{l}\text { Industry and } \\
\text { Innovation }\end{array}$ & 0.790 & $\begin{array}{l}\text { Online communities and } \\
\text { OI }\end{array}$ & not provided & $\begin{array}{l}\text { Analysis and } \\
\text { theorization of the } \\
\text { "community" } \\
\text { construct } \\
\text { applicable to all } \\
\text { innovation-related } \\
\text { studies }\end{array}$ & $\begin{array}{l}\text { Keck Graduate } \\
\text { Institute; } \\
\text { Harvard } \\
\text { Business School }\end{array}$ & USA & $\begin{array}{l}\text { Conceptual/ } \\
\text { theoretical } \\
\text { paper }\end{array}$ \\
\hline 2008 & $\begin{array}{l}\text { Technology } \\
\text { and } \\
\text { Innovation } \\
\text { Management }\end{array}$ & $\begin{array}{l}\text { Lichtenthaler } \\
\text { U. }\end{array}$ & $\begin{array}{l}\text { IEEE } \\
\text { Transactions on } \\
\text { Engineering } \\
\text { Management }\end{array}$ & 0.893 & $\begin{array}{l}\text { Strategic approaches to } \\
\text { technology transactions }\end{array}$ & $\begin{array}{l}\text { external } \\
\text { technology } \\
\text { commercialization } \\
, \quad \text { intellectual } \\
\text { property, OI, } \\
\text { technology } \\
\text { strategy, } \\
\text { technology } \\
\text { transfer }\end{array}$ & $\begin{array}{l}\text { Analysis of the } \\
\text { current state of OI } \\
\text { in practice: despite } \\
\text { its growing } \\
\text { importance, many } \\
\text { firms experience } \\
\text { severe challenges } \\
\text { in actively } \\
\text { managing open } \\
\text { processes. They } \\
\text { often do not benefit } \\
\text { immediately from } \\
\text { establishing } \\
\text { particular } \\
\text { management } \\
\text { mechanisms for } \\
\text { strengthening OI, } \\
\text { such as new } \\
\text { incentive systems }\end{array}$ & $\begin{array}{l}\text { WHU-Otto } \\
\text { Beisheim School } \\
\text { of Management }\end{array}$ & Germany & $\begin{array}{l}\text { Empirical paper } \\
\text { (quantitative } \\
\text { approach- } \\
\text { survey-) }\end{array}$ \\
\hline 2008 & Marketing & $\begin{array}{l}\text { Frow P., } \\
\text { Payne A.F., } \\
\text { Storbacka K. }\end{array}$ & $\begin{array}{l}\text { Journal of the } \\
\text { Academy of } \\
\text { Marketing } \\
\text { Science }\end{array}$ & 2.570 & Value co-creation & $\begin{array}{l}\text { co-creation, } \\
\text { co-production, } \\
\text { service-dominant } \\
\text { logic, value }\end{array}$ & $\begin{array}{l}\text { Exploring the } \\
\text { nature of value } \\
\text { co-creation in the } \\
\text { context of S-D } \\
\text { logic }\end{array}$ & $\begin{array}{l}\text { Australian } \\
\text { School of } \\
\text { Business, } \\
\text { University of } \\
\text { New South } \\
\text { Wales; Nyenrode } \\
\text { Business } \\
\text { Universiteit; The } \\
\text { University of } \\
\text { Sydney }\end{array}$ & $\begin{array}{l}\text { Australia, } \\
\text { The } \\
\text { Netherland } \\
\mathrm{s}\end{array}$ & $\begin{array}{l}\text { Conceptual/ } \\
\text { theoretical } \\
\text { paper }\end{array}$ \\
\hline 2008 & $\begin{array}{l}\text { Technology } \\
\text { and } \\
\text { Innovation } \\
\text { Management }\end{array}$ & $\begin{array}{l}\text { Füller J., von } \\
\text { Hippel E. }\end{array}$ & $\begin{array}{l}\text { Sloan School of } \\
\text { Management } \\
\text { Working Paper }\end{array}$ & - & $\begin{array}{l}\text { First exploratory study } \\
\text { on the topic of } \\
\text { proprietary brands } \\
\text { created by user } \\
\text { communities }\end{array}$ & $\begin{array}{l}\text { community, } \\
\text { brand-premium, } \\
\text { consumer-generate } \\
\text { d brands }\end{array}$ & $\begin{array}{l}\text { User communities } \\
\text { can create strong, } \\
\text { community-owned } \\
\text { brands at a very } \\
\text { low cost. Producers } \\
\text { face a } \\
\text { previously-unexam } \\
\text { ined source of } \\
\text { collaboration; they } \\
\text { may find it } \\
\text { profitable to } \\
\text { co-brand with user } \\
\text { communities }\end{array}$ & $\begin{array}{l}\text { Innsbruck } \\
\text { University } \\
\text { School of } \\
\text { Management; } \\
\text { MIT Sloan } \\
\text { School of } \\
\text { Management }\end{array}$ & $\begin{array}{l}\text { Austria, } \\
\text { USA }\end{array}$ & $\begin{array}{l}\text { Empirical paper } \\
\text { (single case } \\
\text { study) }\end{array}$ \\
\hline 2008 & $\begin{array}{l}\text { Technology } \\
\text { and } \\
\text { Innovation } \\
\text { Management }\end{array}$ & $\begin{array}{ll}\text { Raasch } & \text { C., } \\
\text { Herstatt } & \text { C., } \\
\text { Lock P. } & \end{array}$ & $\begin{array}{l}\text { International } \\
\text { Journal of } \\
\text { Innovation } \\
\text { Management }\end{array}$ & - & $\begin{array}{l}\text { The dynamics of user } \\
\text { innovation }\end{array}$ & $\begin{array}{l}\text { case study, drivers, } \\
\text { dynamic analysis, } \\
\text { impediments, user } \\
\text { communities, user } \\
\text { innovation }\end{array}$ & $\begin{array}{l}\text { The level of user } \\
\text { activity does not } \\
\text { follow a } \\
\text { unidirectional } \\
\text { trend, but develops } \\
\text { depending on } \\
\text { contextual factors: } \\
\text { given a stimulating } \\
\text { setting, user } \\
\text { innovation can be } \\
\text { sustained over long } \\
\text { periods of time }\end{array}$ & $\begin{array}{l}\text { Hamburg } \\
\text { University of } \\
\text { Technology, } \\
\text { Institute of } \\
\text { Technology and } \\
\text { Innovation } \\
\text { Management }\end{array}$ & Germany & $\begin{array}{l}\text { Empirical paper } \\
\text { (single case } \\
\text { study) }\end{array}$ \\
\hline 2008 & $\begin{array}{l}\text { Innovation } \\
\text { Management }\end{array}$ & $\begin{array}{l}\text { Warnke P., } \\
\text { Weber M., } \\
\text { Leitner K.H. }\end{array}$ & $\begin{array}{l}\text { International } \\
\text { journal of } \\
\text { innovation }\end{array}$ & - & $\begin{array}{l}\text { User-centric Innovation } \\
\text { scenarios }\end{array}$ & $\begin{array}{l}\text { foresight, } \\
\text { scenarios, } \\
\text { transition, }\end{array}$ & $\begin{array}{l}\text { Possible future } \\
\text { working } \\
\text { configurations of }\end{array}$ & $\begin{array}{l}\text { Fraunhofer } \\
\text { Institute for } \\
\text { Systems and }\end{array}$ & $\begin{array}{l}\text { Germany, } \\
\text { Austria }\end{array}$ & $\begin{array}{l}\text { Conceptual/ } \\
\text { theoretical } \\
\text { paper }\end{array}$ \\
\hline
\end{tabular}




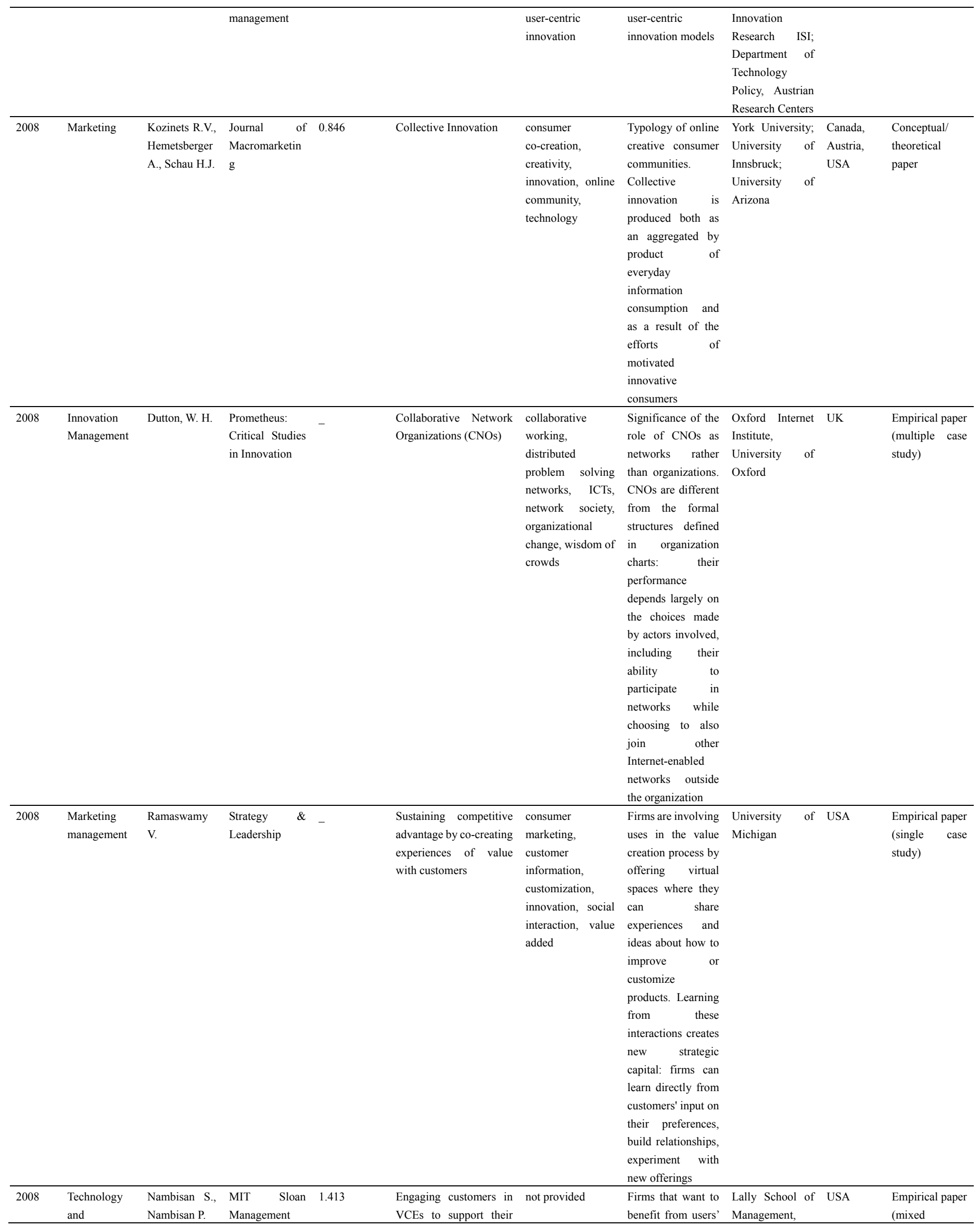




\begin{tabular}{|c|c|c|c|c|c|c|c|c|c|c|}
\hline & $\begin{array}{l}\text { Innovation } \\
\text { Management }\end{array}$ & & Review & & $\begin{array}{l}\text { roles as product } \\
\text { conceptualizers, } \\
\text { designers, marketers }\end{array}$ & & $\begin{array}{l}\text { creativity need to } \\
\text { adopt strategies } \\
\text { that: link their } \\
\text { external innovating } \\
\text { environments with } \\
\text { internal PD teams; } \\
\text { incorporate key } \\
\text { design features into } \\
\text { their VCEs (rating } \\
\text { systems, product } \\
\text { knowledge centers, } \\
\text { exclusive forums, } \\
\text { etc.); embed the } \\
\text { VCEs in CRM } \\
\text { activities }\end{array}$ & $\begin{array}{l}\text { Rensselaer } \\
\text { Polytechnic } \\
\text { Institute; } \\
\text { University at } \\
\text { Albany, State } \\
\text { University } \\
\text { New York }\end{array}$ & & $\begin{array}{l}\text { approach: } \\
\text { quantitative- } \\
\text { extensive } \\
\text { survey-, } \\
\text { qualitative - in } \\
\text { depth } \\
\text { interviews -) }\end{array}$ \\
\hline 2007 & Marketing & $\begin{array}{l}\text { Füller J., } \\
\text { Jawecki G., } \\
\text { Muhlbacher } \\
\text { H. }\end{array}$ & $\begin{array}{l}\text { Journal } \\
\text { Business } \\
\text { Research }\end{array}$ & 1.484 & $\begin{array}{l}\text { Online consumer } \\
\text { participation in the } \\
\text { development of tangible } \\
\text { consumer goods } \\
\text { (basketball shoes) }\end{array}$ & $\begin{array}{l}\text { innovation; online } \\
\text { community; } \\
\text { consumer goods; } \\
\text { knowledge } \\
\text { creation; virtual } \\
\text { consumer } \\
\text { integration; NPD }\end{array}$ & $\begin{array}{l}\text { Insights into the } \\
\text { process and } \\
\text { motives of } \\
\text { innovation creation } \\
\text { within online } \\
\text { communities }\end{array}$ & $\begin{array}{l}\text { Innsbruck } \\
\text { University } \\
\text { School of } \\
\text { Management }\end{array}$ & Austria & $\begin{array}{l}\text { Empirical paper } \\
\text { (qualitative } \\
\text { approach- } \\
\text { netnography: } \\
\text { observations of } \\
\text { community, } \\
\text { qualitative } \\
\text { analyses of the } \\
\text { members' } \\
\text { communication } \\
\text { interviews } \\
\text { with experts -) }\end{array}$ \\
\hline 2007 & Marketing & $\begin{array}{l}\text { Sheth J.N., } \\
\text { Uslay C. }\end{array}$ & $\begin{array}{l}\text { Journal of } \\
\text { Public Policy \& } \\
\text { Marketing }\end{array}$ & 1.348 & $\begin{array}{l}\text { Revised definition of } \\
\text { Marketing by the AMA: } \\
\text { a focus on creating value } \\
\text { through customer } \\
\text { replaces the focus on the } \\
\text { exchange } \\
\text { paradigm }\end{array}$ & $\begin{array}{l}\text { American } \\
\text { Marketing } \\
\text { Association; } \\
\text { definition } \quad \text { of } \\
\text { marketing; } \\
\text { exchange; } \\
\text { marketing } \\
\text { concept; value } \\
\text { cocreation; value } \\
\text { creation }\end{array}$ & 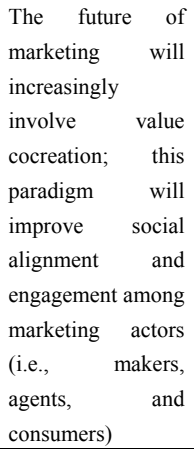 & $\begin{array}{l}\text { Goizueta } \\
\text { Business School, } \\
\text { Emory } \\
\text { University; } \\
\text { Argyros School } \\
\text { of Business and } \\
\text { Economics, } \\
\text { Chapman } \\
\text { University }\end{array}$ & USA & $\begin{array}{l}\text { Conceptual/ } \\
\text { theoretical } \\
\text { paper }\end{array}$ \\
\hline 2007 & $\begin{array}{l}\text { Business } \\
\text { research }\end{array}$ & $\begin{array}{l}\text { Berthon P.R., } \\
\text { Pitt L.F., } \\
\text { McCarthy I., } \\
\text { Kates S.M. }\end{array}$ & $\begin{array}{l}\text { Business } \\
\text { Horizons }\end{array}$ & 1.416 & $\begin{array}{l}\text { Conceptualization of } \\
\text { "creative consumer" vs } \\
\text { "lead-user" }\end{array}$ & $\begin{array}{l}\text { Creative } \\
\text { consumers; firm } \\
\text { stance; strategic } \\
\text { response; } \\
\text { diagnostics }\end{array}$ & $\begin{array}{l}\text { Initial framework } \\
\text { and managerial } \\
\text { approaches to } \\
\text { dealing } r \text { with } \\
\text { creative consumers }\end{array}$ & $\begin{array}{l}\text { Bentley } \\
\text { University; } \\
\text { Simon Fraser } \\
\text { University }\end{array}$ & $\begin{array}{l}\text { USA, } \\
\text { Canada }\end{array}$ & $\begin{array}{l}\text { Conceptual/ } \\
\text { theoretical } \\
\text { paper }\end{array}$ \\
\hline 2007 & Marketing & $\begin{array}{l}\text { Macdonald } \\
\text { E.K, Uncles } \\
\text { M.D. }\end{array}$ & $\begin{array}{l}\text { Journal of } \\
\text { Marketing } \\
\text { Management }\end{array}$ & _- & $\begin{array}{l}\text { Concept of 'savvy } \\
\text { consumers' }\end{array}$ & $\begin{array}{l}\text { Consumer-centrici } \\
\text { ty, consumer } \\
\text { savvy, savvy } \\
\text { consumers, } \\
\text { E-marketing, scale } \\
\text { development }\end{array}$ & $\begin{array}{l}\text { Identification of } \\
\text { key features of } \\
\text { savvy consumers } \\
\text { based on } \\
\text { competency and } \\
\text { empowerment; } \\
\text { SAVVY scale to } \\
\text { measure these } \\
\text { characteristics }\end{array}$ & $\begin{array}{lr}\text { University } & \text { of } \\
\text { New } & \text { South } \\
\text { Wales }\end{array}$ & Australia & $\begin{array}{l}\text { Empirical paper } \\
\text { (mixed } \\
\text { approach: } \\
\text { quantitative- } \\
\text { online survey -, } \\
\text { qualitative- } \\
\text { focus-groups -) }\end{array}$ \\
\hline 2007 & Marketing & $\begin{array}{l}\text { Nambisan S., } \\
\text { Baron R.A. }\end{array}$ & $\begin{array}{ll}\text { Journal of } \\
\text { Interactive }\end{array}$ & 1.000 & $\begin{array}{l}\text { Interactions in VCEs: } \\
\text { implications for value } \\
\text { co-creation and CRM }\end{array}$ & not provided & $\begin{array}{l}\text { Users' interactions } \\
\text { in value } \\
\text { co-creation can be } \\
\text { a strategic source } \\
\text { of value, can shape } \\
\text { their future } \\
\text { participation in the } \\
\text { value co-creation } \\
\text { process and } \\
\text { influence their } \\
\text { attitude to the host } \\
\text { firm: the critical } \\
\text { challenge is in }\end{array}$ & $\begin{array}{l}\text { Lally School of } \\
\text { Management, } \\
\text { Rensselaer } \\
\text { Polytechnic } \\
\text { Institute }\end{array}$ & USA & $\begin{array}{l}\text { Empirical paper } \\
\text { (quantitative } \\
\text { approach- } \\
\text { survey-) }\end{array}$ \\
\hline
\end{tabular}




\begin{tabular}{|c|c|c|c|c|c|c|c|c|c|c|}
\hline & & & & & & & $\begin{array}{l}\text { maintaining a } \\
\text { rewarding } \\
\text { "innovative } \\
\text { experience } \\
\text { environment" } \\
\end{array}$ & & & \\
\hline 2006 & $\begin{array}{l}\text { Technology } \\
\text { and } \\
\text { Innovation } \\
\text { Management }\end{array}$ & Swink M. & $\begin{array}{l}\text { Research } \\
\text { Technology } \\
\text { Management }\end{array}$ & 0.712 & $\begin{array}{l}\text { Product innovation and } \\
\text { supply chain process }\end{array}$ & $\begin{array}{l}\text { product/process } \\
\text { innovation, } \\
\text { collaboration, } \\
\text { organizational } \\
\text { integration }\end{array}$ & $\begin{array}{l}\text { The organization's } \\
\text { innovative } \\
\text { potential is } \\
\text { influenced by its } \\
\text { access to user } \\
\text { intelligence; need } \\
\text { to integrate product } \\
\text { innovation and } \\
\text { supply chain } \\
\text { processes }\end{array}$ & $\begin{array}{l}\text { Michigan State } \\
\text { University }\end{array}$ & USA & $\begin{array}{l}\text { Conceptual/ } \\
\text { theoretical } \\
\text { paper }\end{array}$ \\
\hline 2006 & $\begin{array}{l}\text { Innovation } \\
\text { Management / } \\
\text { NPD }\end{array}$ & $\begin{array}{l}\text { Baldwin C., } \\
\text { Hienerth C., } \\
\text { von Hippel E. }\end{array}$ & Research Policy & 2.850 & $\begin{array}{ll}\text { How user } & \text { innovations } \\
\text { become } & \text { commercial } \\
\text { products } & \end{array}$ & $\begin{array}{l}\text { user innovation, } \\
\text { communities, } \\
\text { dominant design, } \\
\text { industry evolution, } \\
\text { real options }\end{array}$ & $\begin{array}{l}\text { Model of the } \\
\text { pathways traversed } \\
\text { as user innovations } \\
\text { are transformed } \\
\text { into commercial } \\
\text { products in the } \\
\text { rodeo kayak } \\
\text { industry }\end{array}$ & $\begin{array}{l}\text { Harvard } \\
\text { Business School; } \\
\text { Copenhagen } \\
\text { Business School; } \\
\text { MIT Sloan } \\
\text { School of } \\
\text { Management }\end{array}$ & $\begin{array}{l}\text { USA, } \\
\text { Denmark }\end{array}$ & $\begin{array}{l}\text { Empirical paper } \\
\text { (qualitative } \\
\text { approach - case } \\
\text { history -) }\end{array}$ \\
\hline 2006 & $\begin{array}{l}\text { Innovation } \\
\text { Management / } \\
\text { NPD }\end{array}$ & $\begin{array}{l}\text { von Hippel E., } \\
\text { von Krogh G. }\end{array}$ & $\begin{array}{l}\text { R\&D } \\
\text { Management }\end{array}$ & - & $\begin{array}{l}\text { Private-collective model } \\
\text { of innovation }\end{array}$ & not provided & $\begin{array}{l}\text { Free revealing of } \\
\text { the detailed } \\
\text { workings, } \\
\text { information and } \\
\text { knowledge } \\
\text { regarding of novel } \\
\text { products is a } \\
\text { central feature of } \\
\text { OI. The } \\
\text { phenomenon } \\
\text { suggests that an } \\
\text { alternative model } \\
\text { to private } \\
\text { innovation model } \\
\text { exists }\end{array}$ & $\begin{array}{l}\text { Sloan School of } \\
\text { Management, } \\
\text { MIT; ETH } \\
\text { Zurich, } \\
\text { Department of } \\
\text { Management } \\
\text { Technology } \\
\text { Economics }\end{array}$ & $\begin{array}{l}\text { USA, } \\
\text { Switzerlan } \\
\text { d }\end{array}$ & $\begin{array}{l}\text { Conceptual/ } \\
\text { theoretical } \\
\text { paper }\end{array}$ \\
\hline 2006 & $\begin{array}{l}\text { Innovation } \\
\text { Management / } \\
\text { NPD }\end{array}$ & $\begin{array}{l}\text { Piller F.T., } \\
\text { Walcher D. }\end{array}$ & $\begin{array}{l}\text { R\&D } \\
\text { Management }\end{array}$ & - & $\begin{array}{l}\text { Internet-based toolkits } \\
\text { for idea competitions } \\
\text { (TIC) as a method of } \\
\text { identifying lead users }\end{array}$ & not provided & $\begin{array}{l}\text { The performance } \\
\text { of a TIC is } \\
\text { influenced by: } \\
\text { design of the user } \\
\text { interface, } \\
\text { procedure of the } \\
\text { idea formulation, } \\
\text { methods for } \\
\text { pre-screening the } \\
\text { ideas }\end{array}$ & $\begin{array}{l}\text { TUM Business } \\
\text { School, } \\
\text { Technische } \\
\text { Universität } \\
\text { München; MIT } \\
\text { Sloan School of } \\
\text { Management }\end{array}$ & $\begin{array}{l}\text { Germany, } \\
\text { USA }\end{array}$ & $\begin{array}{l}\text { Empirical paper } \\
\text { (single case } \\
\text { study) }\end{array}$ \\
\hline 2006 & $\begin{array}{l}\text { Technology } \\
\text { and } \\
\text { Innovation } \\
\text { Management }\end{array}$ & $\begin{array}{l}\text { Lettl C., } \\
\text { Herstatt C., } \\
\text { Gemuenden } \\
\text { H.G. }\end{array}$ & $\begin{array}{l}\text { International } \\
\text { Journal of } \\
\text { Technology } \\
\text { Management }\end{array}$ & 0.564 & $\begin{array}{l}\text { Learning from users for } \\
\text { radical innovation }\end{array}$ & $\begin{array}{l}\text { innovation } \\
\text { management, } \\
\text { medical } \\
\text { technology, NPD, } \\
\text { PI, radical } \\
\text { innovation, } \\
\text { technology } \\
\text { management, } \\
\text { user-driven } \\
\text { innovation }\end{array}$ & $\begin{array}{l}\text { By interacting with } \\
\text { users with a } \\
\text { specific set of } \\
\text { characteristics, } \\
\text { firms can obtain } \\
\text { substantial } \\
\text { contributions to the } \\
\text { development of } \\
\text { radical innovations }\end{array}$ & $\begin{array}{l}\text { Berlin University } \\
\text { of Technology; } \\
\text { Hamburg } \\
\text { University of } \\
\text { Technology }\end{array}$ & Germany & $\begin{array}{l}\text { Empirical paper } \\
\text { (multiple case } \\
\text { study) }\end{array}$ \\
\hline 2006 & $\begin{array}{l}\text { Innovation } \\
\text { Management / } \\
\text { NPD }\end{array}$ & $\begin{array}{l}\text { Franke N., } \\
\text { von Hippel E., } \\
\text { Schreier M. }\end{array}$ & $\begin{array}{l}\text { Journal of } \\
\text { Product } \\
\text { Innovation } \\
\text { Management }\end{array}$ & 1.572 & $\begin{array}{l}\text { Exploiting the value of } \\
\text { lead- } \\
\text { user innovations for } \\
\text { commercial advantage }\end{array}$ & 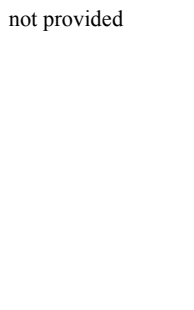 & $\begin{array}{l}\text { Product } \\
\text { modification and } \\
\text { development has } \\
\text { been found to be a } \\
\text { common user } \\
\text { behavior in many } \\
\text { fields: it is } \\
\text { important to find } \\
\text { ways to selectively }\end{array}$ & $\begin{array}{l}\text { Vienna } \\
\text { University of } \\
\text { Economics and } \\
\text { BA; MIT Sloan } \\
\text { School of } \\
\text { Management }\end{array}$ & $\begin{array}{l}\text { Austria, } \\
\text { USA }\end{array}$ & $\begin{array}{l}\text { Empirical paper } \\
\text { (mixed } \\
\text { approach: } \\
\text { quantitative- } \\
\text { survey-, } \\
\text { qualitatitive- } \\
\text { workshops-) }\end{array}$ \\
\hline
\end{tabular}




\begin{tabular}{|c|c|c|c|c|c|c|c|c|c|c|}
\hline & & & & & & & $\begin{array}{l}\text { identify the user } \\
\text { innovations for } \\
\text { commercially } \\
\text { attractive } \\
\end{array}$ & & & \\
\hline 2006 & $\begin{array}{l}\text { Innovation } \\
\text { Management / } \\
\text { NPD }\end{array}$ & $\begin{array}{ll}\text { Ogawa } & \text { S., } \\
\text { Piller F.T. } & \end{array}$ & $\begin{array}{l}\text { MIT Sloan } \\
\text { Management } \\
\text { Review }\end{array}$ & 1.413 & $\begin{array}{l}\text { Integrate customers into } \\
\text { NPD to reduce risks }\end{array}$ & not provided & $\begin{array}{l}\text { The use of } \\
\text { collective customer } \\
\text { commitment can be } \\
\text { particularly } \\
\text { effective for two } \\
\text { types of situations: } \\
\text { (1) testing } \\
\text { innovative } \\
\text { products for which } \\
\text { little experience } \\
\text { exists and market } \\
\text { research is fuzzy; } \\
\text { (2) developing for } \\
\text { small and very } \\
\text { heterogeneous } \\
\text { market segments - } \\
\text { it occurs with } \\
\text { increasing } \\
\text { frequency because } \\
\text { of fast-changing } \\
\text { market trends and } \\
\text { diverse customer } \\
\text { needs - }\end{array}$ & $\begin{array}{l}\text { Graduate School } \\
\text { of BA at Kobe } \\
\text { University in } \\
\text { Kobe; TUM } \\
\text { Business School, } \\
\text { Technische } \\
\text { Universität } \\
\text { München }\end{array}$ & $\begin{array}{l}\text { Japan, } \\
\text { Germany }\end{array}$ & $\begin{array}{l}\text { Empirical paper } \\
\text { (multiple case } \\
\text { study) }\end{array}$ \\
\hline 2006 & Marketing & $\begin{array}{lr}\text { Pitt } & \text { L.F., } \\
\text { Watson } & \text { R.T., } \\
\text { Berthon } & \text { P., } \\
\text { Wynn } & \text { D., } \\
\text { Zinkhan G. }\end{array}$ & $\begin{array}{l}\text { Journal of the } \\
\text { Academy of } \\
\text { Marketing } \\
\text { Science }\end{array}$ & 2.570 & $\begin{array}{l}\text { OS principles and } \\
\text { corporate branding } \\
\text { revision towards a } \\
\text { coproducer perspective }\end{array}$ & $\begin{array}{l}\text { brands, open } \\
\text { source, brand } \\
\text { typology, } \\
\text { prosumer, brand } \\
\text { function, brand } \\
\text { evolution }\end{array}$ & $\begin{array}{l}\text { Typology of brand } \\
\text { aspects that can be } \\
\text { "open" or "closed": } \\
\text { physical, textual, } \\
\text { meaning, } \\
\text { experience. } \\
\text { Open-source } \\
\text { represents a final } \\
\text { phase in the } \\
\text { evolution of } \\
\text { corporate brands } \\
\text { from closed to } \\
\text { open brands }\end{array}$ & $\begin{array}{l}\text { Simon Fraser } \\
\text { University; } \\
\text { Bentley College; } \\
\text { University of } \\
\text { Georgia }\end{array}$ & $\begin{array}{l}\text { Canada, } \\
\text { USA }\end{array}$ & $\begin{array}{l}\text { Conceptual/ } \\
\text { theoretical } \\
\text { paper }\end{array}$ \\
\hline 2005 & Marketing & $\begin{array}{l}\text { Sawhney M., } \\
\text { Verona G., } \\
\text { Prandelli E. }\end{array}$ & $\begin{array}{l}\text { Journal of } \\
\text { Interactive } \\
\text { Marketing }\end{array}$ & 1.000 & $\begin{array}{l}\text { Internet as a platform for } \\
\text { customer engagement in } \\
\text { PD }\end{array}$ & not provided & $\begin{array}{l}\text { Distinctive } \\
\text { capabilities of the } \\
\text { Internet as a } \\
\text { platform for } \\
\text { customer } \\
\text { engagement }\end{array}$ & $\begin{array}{l}\text { Kellogg School } \\
\text { of Management } \\
\text { Northwestern } \\
\text { University; } \\
\text { Bocconi } \\
\text { University }\end{array}$ & USA, Italy & $\begin{array}{l}\text { Empirical paper } \\
\text { (multiple case } \\
\text { study) }\end{array}$ \\
\hline 2005 & $\begin{array}{l}\text { Innovation } \\
\text { Management / } \\
\text { NPD }\end{array}$ & Jeppesen L.B. & $\begin{array}{l}\text { Journal of } \\
\text { Product } \\
\text { Innovation } \\
\text { Management }\end{array}$ & 1.572 & $\begin{array}{l}\text { Relation between the } \\
\text { employment of user } \\
\text { toolkits and the need for } \\
\text { firms to support } \\
\text { consumers }\end{array}$ & not provided & $\begin{array}{l}\text { Consumer-to-cons } \\
\text { umer (C2C) } \\
\text { interaction reduces } \\
\text { the amount of } \\
\text { resources that the } \\
\text { firm needs to } \\
\text { dedicate to support } \\
\text { consumers using } \\
\text { toolkits }\end{array}$ & $\begin{array}{l}\text { Copenhagen } \\
\text { Business School }\end{array}$ & Denmark & $\begin{array}{l}\text { Empirical paper } \\
\text { (mixed } \\
\text { approach: } \\
\text { survey, single } \\
\text { case study) }\end{array}$ \\
\hline 2005 & NPD & $\begin{array}{l}\text { Tietz R., } \\
\text { Morrison P.D., } \\
\text { Luthje C., } \\
\text { Herstatt C. }\end{array}$ & $\begin{array}{l}\text { International } \\
\text { Journal of } \\
\text { Product } \\
\text { Development }\end{array}$ & - & User-innovation process & $\begin{array}{l}\text { user innovation, } \\
\text { lead-user, PD, } \\
\text { innovation } \\
\text { process, end-users, } \\
\text { consumer markets, } \\
\text { user inventors, } \\
\text { idea generation, } \\
\text { consumer goods, } \\
\text { novel products, } \\
\text { kite surfing, idea } \\
\text { realisation, } \\
\text { product design, }\end{array}$ & $\begin{array}{l}\text { Identification of a } \\
\text { user-inventor } \\
\text { approach sequence, } \\
\text { consisting of idea } \\
\text { generation and } \\
\text { realization. } \\
\text { Manufacturer can } \\
\text { profit from more } \\
\text { closely observing } \\
\text { such user activities } \\
\text { to better } \\
\text { understanding of }\end{array}$ & $\begin{array}{l}\text { Hamburg } \\
\text { University of } \\
\text { Technology } \\
\text { (TUHH); School } \\
\text { of Marketing, } \\
\text { The University } \\
\text { of New South } \\
\text { Wales; Institute } \\
\text { of Technology } \\
\text { and Innovation } \\
\text { Management, } \\
\text { Philipps-Univers }\end{array}$ & $\begin{array}{l}\text { Germany, } \\
\text { Australia }\end{array}$ & $\begin{array}{l}\text { Empirical paper } \\
\text { (mixed } \\
\text { approach: } \\
\text { quantitative- } \\
\text { survey-, } \\
\text { qualitative- } \\
\text { interviews -) }\end{array}$ \\
\hline
\end{tabular}




\begin{tabular}{|c|c|c|c|c|c|c|c|c|c|c|}
\hline & & & & & & product innovation & $\begin{array}{l}\text { tacit needs; to } \\
\text { collect user ideas at } \\
\text { very low tariffs; to } \\
\text { increase reputation } \\
\text { as a customer-close } \\
\text { firm; to learn about } \\
\text { the adequacy of } \\
\text { solutions, } \\
\text { preventing } \\
\text { development of } \\
\text { inadequate } \\
\text { solutions }\end{array}$ & ity Marburg & & \\
\hline 2004 & Marketing & $\begin{array}{l}\text { Prahalad C. } \\
\text { K., } \\
\text { Ramaswamy } \\
\text { V. }\end{array}$ & $\begin{array}{l}\text { Journal of } \\
\text { Interactive } \\
\text { Marketing }\end{array}$ & 1.000 & $\begin{array}{l}\text { Co-creation as the base } \\
\text { of value }\end{array}$ & not provided & $\begin{array}{l}\text { High-quality } \\
\text { interactions that } \\
\text { enable customers } \\
\text { to co-create unique } \\
\text { experiences with } \\
\text { the company are } \\
\text { the key to } \\
\text { unlocking new } \\
\text { sources } \\
\text { competitive } \\
\text { advantage }\end{array}$ & $\begin{array}{l}\text { University of } \\
\text { Michigan } \\
\text { Business School }\end{array}$ & USA & $\begin{array}{l}\text { Conceptual/ } \\
\text { theoretical } \\
\text { paper }\end{array}$ \\
\hline 2004 & Management & $\begin{array}{l}\text { Prahalad C. } \\
\text { K., } \\
\text { Ramaswamy } \\
\text { V. }\end{array}$ & $\begin{array}{l}\text { Strategy \& } \\
\text { Leadership }\end{array}$ & _- & $\begin{array}{l}\text { Co-creation with } \\
\text { consumers }\end{array}$ & $\begin{array}{l}\text { Competitive } \\
\text { advantage, } \\
\text { organizations, } \\
\text { value added }\end{array}$ & $\begin{array}{l}\text { The traditional } \\
\text { system of } \\
\text { company-centric } \\
\text { value creation is } \\
\text { becoming obsolete. } \\
\text { DART model for } \\
\text { managing } \\
\text { co-creation of } \\
\text { value process and } \\
\text { to understand it } \\
\text { through: dialogue; } \\
\text { access; risk } \\
\text { assessment; } \\
\text { transparency }\end{array}$ & $\begin{array}{l}\text { University of } \\
\text { Michigan } \\
\text { Business School }\end{array}$ & USA & $\begin{array}{l}\text { Conceptual/ } \\
\text { theoretical } \\
\text { paper }\end{array}$ \\
\hline 2004 & $\begin{array}{l}\text { Innovation } \\
\text { Management / } \\
\text { NPD }\end{array}$ & $\begin{array}{l}\text { Franke } \quad \text { N., } \\
\text { Piller F. }\end{array}$ & $\begin{array}{l}\text { Journal of } \\
\text { Product } \\
\text { Innovation } \\
\text { Management }\end{array}$ & 1.572 & $\begin{array}{l}\text { Value created by toolkits } \\
\text { for user innovation and } \\
\text { design }\end{array}$ & not provided & $\begin{array}{l}\text { Toolkit's ability to } \\
\text { allow customers to } \\
\text { customize products } \\
\text { to suit their } \\
\text { preferences and } \\
\text { creates value for } \\
\text { them in a B2C } \\
\text { setting; consumers } \\
\text { are also willing to } \\
\text { pay a price } \\
\text { premium }\end{array}$ & $\begin{array}{l}\text { Vienna } \\
\text { University of } \\
\text { Business }\end{array}$ & Austria & $\begin{array}{l}\text { Empirical paper } \\
\text { (single case } \\
\text { study) }\end{array}$ \\
\hline 2003 & $\begin{array}{l}\text { Technology } \\
\text { and } \\
\text { Innovation } \\
\text { Management }\end{array}$ & Brockhoff K. & $\begin{array}{l}\text { International } \\
\text { Journal of } \\
\text { Technology } \\
\text { Management }\end{array}$ & 0.564 & $\begin{array}{l}\text { Customers' perspectives } \\
\text { of involvement in NPD }\end{array}$ & $\begin{array}{l}\text { cooperation, } \\
\text { customer } \\
\text { involvement, } \\
\text { innovation, NPD }\end{array}$ & $\begin{array}{l}\text { Better } \\
\text { understanding of } \\
\text { customer } \\
\text { involvement from } \\
\text { the suppliers' side } \\
\end{array}$ & $\begin{array}{l}\text { WHU - Otto } \\
\text { Beisheim School } \\
\text { of Management }\end{array}$ & Germany & $\begin{array}{l}\text { Conceptual/ } \\
\text { theoretical } \\
\text { paper }\end{array}$ \\
\hline 2003 & Management & $\begin{array}{l}\text { Chesbrough } \\
\text { H. }\end{array}$ & $\begin{array}{l}\text { MIT Sloan } \\
\text { Management } \\
\text { Review }\end{array}$ & 1.413 & $\begin{array}{l}\text { Rethinking the } \\
\text { fundamental ways in } \\
\text { which firms generate } \\
\text { ideas and bring them to } \\
\text { market }\end{array}$ & innovation, OI & $\begin{array}{l}\text { OI embraces } \\
\text { external ideas and } \\
\text { knowledge in } \\
\text { conjunction with } \\
\text { internal R\&D; this } \\
\text { change offers novel } \\
\text { ways to create } \\
\text { value }\end{array}$ & $\begin{array}{l}\text { Harvard } \\
\text { Business School } \\
\text { Boston }\end{array}$ & USA & $\begin{array}{l}\text { Conceptual/ } \\
\text { theoretical } \\
\text { paper }\end{array}$ \\
\hline 2002 & $\begin{array}{l}\text { Innovation } \\
\text { Management / } \\
\text { NPD }\end{array}$ & $\begin{array}{l}\text { Dahan E., } \\
\text { Hauser J.R. }\end{array}$ & $\begin{array}{l}\text { Journal of } \\
\text { Product } \\
\text { Innovation } \\
\text { Management } \\
\end{array}$ & 1.572 & $\begin{array}{l}\text { PD process and } \\
\text { customer inputs }\end{array}$ & not provided & $\begin{array}{l}\text { Methods of } \\
\text { customer input in } \\
\text { the various stages } \\
\text { of PD }\end{array}$ & $\begin{array}{l}\text { Sloan School of } \\
\text { Management, } \\
\text { MIT }\end{array}$ & USA & $\begin{array}{l}\text { Methodological } \\
\text { paper }\end{array}$ \\
\hline 2002 & $\begin{array}{l}\text { Innovation } \\
\text { Management/ }\end{array}$ & Danneels E. & $\begin{array}{l}\text { Strategic } \\
\text { Management }\end{array}$ & 3.367 & $\begin{array}{l}\text { Dynamics of product } \\
\text { innovation: }\end{array}$ & $\begin{array}{l}\text { dynamic } \\
\text { capabilities, firm }\end{array}$ & $\begin{array}{l}\text { Based on the } \\
\text { notion that new }\end{array}$ & $\begin{array}{l}\text { Worcester } \\
\text { Polytechnic }\end{array}$ & USA & $\begin{array}{l}\text { Empirical paper } \\
\text { (multiple case }\end{array}$ \\
\hline
\end{tabular}




\begin{tabular}{|c|c|c|c|c|c|c|c|c|c|c|}
\hline & NPD & & Journal & & $\begin{array}{l}\text { alternatives and role of } \\
\text { consumers }\end{array}$ & $\begin{array}{l}\text { competences, } \\
\text { organizational } \\
\text { learning, path } \\
\text { dependency, } \\
\text { product innovation }\end{array}$ & $\begin{array}{l}\text { products are } \\
\text { created by linking } \\
\text { competences } \\
\text { relating to } \\
\text { technologies and } \\
\text { customers, a } \\
\text { typology classifies } \\
\text { new product } \\
\text { projects based on } \\
\text { whether a new } \\
\text { product can draw } \\
\text { on existing } \\
\text { competences, or } \\
\text { whether it requires } \\
\text { competences the } \\
\text { firm does not yet } \\
\text { have }\end{array}$ & Institute & & study) \\
\hline 2002 & Management & Nambisan S. & $\begin{array}{l}\text { Academy of } \\
\text { Management } \\
\text { Review }\end{array}$ & 7.895 & $\begin{array}{l}\text { Virtual customer } \\
\text { environments design for } \\
\text { NPD }\end{array}$ & not provided & $\begin{array}{l}\text { Propositions } \\
\text { relating customer } \\
\text { environment } \\
\text { design elements to } \\
\text { successful value } \\
\text { creation and NPD } \\
\text { success }\end{array}$ & $\begin{array}{l}\text { Rensselaer } \\
\text { Polytechnic } \\
\text { Institute, Lally } \\
\text { School of } \\
\text { Management }\end{array}$ & USA & $\begin{array}{l}\text { Conceptual/ } \\
\text { theoretical } \\
\text { paper }\end{array}$ \\
\hline 2002 & $\begin{array}{l}\text { Technology } \\
\text { and } \\
\text { Innovation } \\
\text { Management }\end{array}$ & $\begin{array}{l}\text { von Hippel E., } \\
\text { Katz R. }\end{array}$ & $\begin{array}{l}\text { Management } \\
\text { Science }\end{array}$ & 1.859 & $\begin{array}{l}\text { Transferring } \\
\text { need-related aspects of } \\
\text { product development to } \\
\text { users }\end{array}$ & $\begin{array}{l}\text { user Innovation, } \\
\text { toolkits, mass } \\
\text { customization, PD }\end{array}$ & $\begin{array}{l}\text { Exploring toolkits } \\
\text { for user innovation } \\
\text { and explaining why } \\
\text { and how they work }\end{array}$ & $\begin{array}{l}\text { Sloan School of } \\
\text { Management, } \\
\text { MIT; } \\
\text { Northeastern } \\
\text { University, } \\
\text { Boston } \\
\end{array}$ & USA & $\begin{array}{l}\text { Conceptual/ } \\
\text { theoretical } \\
\text { paper }\end{array}$ \\
\hline 2002 & $\begin{array}{l}\text { Business } \\
\text { research }\end{array}$ & $\begin{array}{l}\text { Thomke S., } \\
\text { von Hippel E. }\end{array}$ & $\begin{array}{l}\text { Harvard } \\
\text { Business } \\
\text { Review }\end{array}$ & 1.519 & Customers as Innovators & not provided & $\begin{array}{l}\text { Basic principles for } \\
\text { industries } \\
\text { undergoing } \\
\text { transformations } \\
\text { about the location } \\
\text { where value is } \\
\text { created }\end{array}$ & $\begin{array}{l}\text { Harvard } \\
\text { Business School; } \\
\text { Sloan School of } \\
\text { Management, } \\
\text { MIT }\end{array}$ & USA & $\begin{array}{l}\text { Conceptual/ } \\
\text { theoretical } \\
\text { paper }\end{array}$ \\
\hline 2002 & $\begin{array}{l}\text { Innovation } \\
\text { Management / } \\
\text { NPD }\end{array}$ & $\begin{array}{l}\text { Lilien G.L., } \\
\text { Morrison P.D., } \\
\text { Searls K., } \\
\text { Sonnack M., } \\
\text { von Hippel E. }\end{array}$ & $\begin{array}{l}\text { Management } \\
\text { Science }\end{array}$ & 1.859 & $\begin{array}{l}\text { Lead-user } \\
\text { idea-generation process } \\
\text { for NPD }\end{array}$ & $\begin{array}{l}\text { new product } \\
\text { development, lead } \\
\text { users, idea } \\
\text { generation }\end{array}$ & $\begin{array}{l}\text { The LU } \\
\text { idea-generation } \\
\text { method does } \\
\text { appear to generate } \\
\text { better forecast sales } \\
\text { than traditional } \\
\text { methods }\end{array}$ & $\begin{array}{l}\text { Smeal College of } \\
\text { BA, } \\
\text { Pennsylvania } \\
\text { State University; } \\
\text { University of } \\
\text { New South } \\
\text { Wales; } \\
\text { MIT Sloan } \\
\text { School of } \\
\text { Management } \\
\end{array}$ & $\begin{array}{l}\text { USA, } \\
\text { Australia }\end{array}$ & $\begin{array}{l}\text { Empirical paper } \\
\text { (single case } \\
\text { study) }\end{array}$ \\
\hline 2001 & $\begin{array}{l}\text { Innovation } \\
\text { Management / } \\
\text { NPD }\end{array}$ & von Hippel E. & $\begin{array}{l}\text { Journal of } \\
\text { Product } \\
\text { Innovation } \\
\text { Management }\end{array}$ & 1.572 & $\begin{array}{l}\text { User toolkits for } \\
\text { innovation vs traditional, } \\
\text { manufacturer methods }\end{array}$ & not provided & $\begin{array}{l}\text { User toolkits for } \\
\text { innovation can be } \\
\text { applied: } \\
\text {-to both physical } \\
\text { and information } \\
\text { goods, } \\
\text {-to all types of } \\
\text { products where } \\
\text { heterogeneity of } \\
\text { user demand makes } \\
\text { customization } \\
\text { valuable to buyers }\end{array}$ & $\begin{array}{l}\text { Sloan School of } \\
\text { Management, } \\
\text { MIT }\end{array}$ & USA & $\begin{array}{l}\text { Conceptual/ } \\
\text { theoretical } \\
\text { paper }\end{array}$ \\
\hline 2000 & $\begin{array}{l}\text { Business } \\
\text { research }\end{array}$ & $\begin{array}{l}\text { Prahalad C.K., } \\
\text { Ramaswamy } \\
\text { V. }\end{array}$ & $\begin{array}{l}\text { Harvard } \\
\text { Business } \\
\text { Review }\end{array}$ & 1.519 & $\begin{array}{l}\text { Co-Opting Customer } \\
\text { Competence }\end{array}$ & not provided & $\begin{array}{l}\text { In a market in } \\
\text { which } \\
\text { technology-enable } \\
\mathrm{d} \text { consumers can } \\
\text { dialogue with } \\
\text { manufacturers, } \\
\text { firms have to } \\
\text { recognize that they }\end{array}$ & $\begin{array}{l}\text { University of } \\
\text { Michigan } \\
\text { Business School }\end{array}$ & USA & $\begin{array}{l}\text { Conceptual/ } \\
\text { theoretical } \\
\text { paper }\end{array}$ \\
\hline
\end{tabular}




\begin{tabular}{|c|c|c|c|c|c|c|c|c|c|}
\hline & & & & & & & $\begin{array}{l}\text { are becoming } \\
\text { partners in creating } \\
\text { value. } \\
\text { The shifting role of } \\
\text { the consumers } \\
\text { affects the notion } \\
\text { of a firm's core } \\
\text { competencies. } \\
\text { Where previously, } \\
\text { businesses learned } \\
\text { to draw on the } \\
\text { competencies and } \\
\text { resources of their } \\
\text { business partners } \\
\text { and suppliers to } \\
\text { compete } \\
\text { effectively, they } \\
\text { must now include } \\
\text { consumers as part } \\
\text { of the extended } \\
\text { enterprise. } \\
\text { Managers have to } \\
\text { engage them in: } \\
\text {-active, explicit, } \\
\text { and ongoing } \\
\text { dialogue; } \\
\text {-mobilize } \\
\text { consumers' } \\
\text { communities; } \\
\text {-manage customer } \\
\text { diversity; } \\
\text {-engage them in } \\
\text { co-creating } \\
\text { personalized } \\
\text { experiences }\end{array}$ & & \\
\hline 1998 & Management & $\begin{array}{l}\text { Slater S. F., } \\
\text { Narver J.C. }\end{array}$ & $\begin{array}{l}\text { Strategic } \\
\text { Management } \\
\text { Journal }\end{array}$ & 3.367 & $\begin{array}{l}\text { Customer-led vs } \\
\text { market-oriented } \\
\text { business approach }\end{array}$ & $\begin{array}{l}\text { market orientation, } \\
\text { innovation, } \\
\text { corporate culture, } \\
\text { discontinuous } \\
\text { change }\end{array}$ & $\begin{array}{lr}\text { A } & \text { market } \\
\text { orientation } & \text { is } \\
\text { essential } & \text { to } \\
\text { innovation process } \\
\text { success } \\
\end{array}$ & $\begin{array}{l}\text { University of USA } \\
\text { Washington }\end{array}$ & $\begin{array}{l}\text { Conceptual/ } \\
\text { theoretical } \\
\text { paper }\end{array}$ \\
\hline 1995 & $\begin{array}{l}\text { Technology } \\
\text { and } \\
\text { Innovation } \\
\text { Management }\end{array}$ & $\begin{array}{l}\text { Gales L., } \\
\text { Mansour-Cole } \\
\text { D. }\end{array}$ & $\begin{array}{l}\text { Journal of } \\
\text { Engineering and } \\
\text { Technology } \\
\text { Management }\end{array}$ & 0.967 & $\begin{array}{l}\text { User involvement in } \\
\text { innovation projects }\end{array}$ & $\begin{array}{l}\text { user involvement, } \\
\text { information } \\
\text { processing, } \\
\text { innovation } \\
\text { management }\end{array}$ & $\begin{array}{l}\text { User involvement } \\
\text { increases as } \\
\text { projects progress } \\
\text { from idea } \\
\text { generation to } \\
\text { commercialization } \\
\text { and with respect to } \\
\text { uncertainty }\end{array}$ & $\begin{array}{l}\text { College of BA, USA } \\
\text { University of } \\
\text { Cincinnati }\end{array}$ & $\begin{array}{l}\text { Empirical paper } \\
\text { (quantitative } \\
\text { approach: } \\
\text { survey) }\end{array}$ \\
\hline 1994 & $\begin{array}{l}\text { Marketing } \\
\text { management }\end{array}$ & Day G.S. & $\begin{array}{l}\text { California } \\
\text { Management } \\
\text { Review }\end{array}$ & 1.667 & $\begin{array}{l}\text { Market-driven firms and } \\
\text { processes }\end{array}$ & not provided & $\begin{array}{l}\text { Understanding } \\
\text { ways to learn about } \\
\text { markets: step } \\
\text { analysis and } \\
\text { method suggested } \\
\end{array}$ & $\begin{array}{l}\text { Wharton School, USA } \\
\text { University of } \\
\text { Pennsylvania }\end{array}$ & $\begin{array}{l}\text { Conceptual/ } \\
\text { theoretical } \\
\text { paper }\end{array}$ \\
\hline 1990 & $\begin{array}{l}\text { Innovation } \\
\text { Management / } \\
\text { NPD }\end{array}$ & $\begin{array}{l}\text { Stuermer M., } \\
\text { Spaeth S., Von } \\
\text { Krogh G. }\end{array}$ & $\begin{array}{l}\text { R\&D } \\
\text { Management }\end{array}$ & _ & $\begin{array}{l}\text { Private-collective } \\
\text { innovation }\end{array}$ & not provided & $\begin{array}{l}\text { Managers should } \\
\text { investigate ways to } \\
\text { implement } \\
\text { private-collective } \\
\text { innovation: sharing } \\
\text { development costs } \\
\text { and enabling } \\
\text { contributions from } \\
\text { third parties are } \\
\text { reasons why the } \\
\text { model is attractive. } \\
\text { Its implementation } \\
\text { is associated with } \\
\text { benefits, costs and }\end{array}$ & $\begin{array}{l}\text { ETH Zurich, Switzerlan } \\
\text { Department of } \mathrm{d} \\
\text { Management } \\
\text { Technology and } \\
\text { Economics }\end{array}$ & $\begin{array}{l}\text { Empirical paper } \\
\text { (single case } \\
\text { study) }\end{array}$ \\
\hline
\end{tabular}




\begin{tabular}{|c|c|c|c|c|c|c|c|c|c|c|}
\hline & & & & & & & $\begin{array}{l}\text { strategies to } \\
\text { mitigate these }\end{array}$ & & & \\
\hline 1986 & $\begin{array}{l}\text { Innovation } \\
\text { Management / } \\
\text { NPD }\end{array}$ & von Hippel E. & $\begin{array}{l}\text { Management } \\
\text { Science }\end{array}$ & 1.859 & Lead Users & $\begin{array}{l}\text { innovation } \\
\text { management, } \\
\text { marketing - new } \\
\text { products, R\&D }\end{array}$ & $\begin{array}{l}\text { How lead users can } \\
\text { be identified; how } \\
\text { their perceptions } \\
\text { and preferences } \\
\text { can be incorporated } \\
\text { into marketing } \\
\text { research analyses } \\
\text { of needs for new } \\
\text { products }\end{array}$ & $\begin{array}{l}\text { Sloan School of } \\
\text { Management, } \\
\text { MIT }\end{array}$ & USA & $\begin{array}{l}\text { Conceptual/ } \\
\text { theoretical } \\
\text { paper }\end{array}$ \\
\hline 1983 & $\begin{array}{l}\text { Innovation } \\
\text { Management / } \\
\text { NPD }\end{array}$ & von Hippel E. & $\begin{array}{l}\text { MIT Sloan } \\
\text { School of } \\
\text { Management } \\
\text { Working Paper }\end{array}$ & - & $\begin{array}{l}\text { Novel product concepts } \\
\text { from lead users }\end{array}$ & not provided & 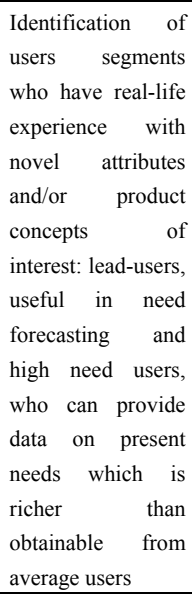 & $\begin{array}{l}\text { Sloan School of } \\
\text { Management, } \\
\text { MIT }\end{array}$ & USA & $\begin{array}{l}\text { Conceptual/ } \\
\text { theoretical } \\
\text { paper }\end{array}$ \\
\hline 1976 & $\begin{array}{l}\text { Technology } \\
\text { and } \\
\text { Innovation } \\
\text { Management }\end{array}$ & 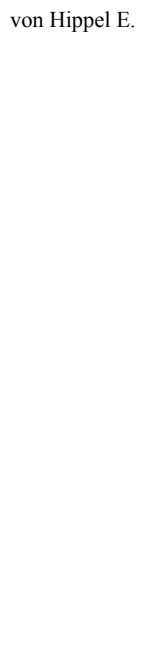 & Research Policy & 2.850 & $\begin{array}{l}\text { Role of users in the } \\
\text { scientific instrument } \\
\text { innovation process }\end{array}$ & not provided & $\begin{array}{l}\text { The } \\
\text { user-dominated } \\
\text { innovation pattern } \\
\text { observed in } \\
\text { scientific } \\
\text { instruments may } \\
\text { play a major role in } \\
\text { numerous other } \\
\text { sectors. In the } \\
\text { context analyzed, } \\
\text { the role of the } \\
\text { manufacturer was } \\
\text { restricted to the } \\
\text { performance of } \\
\text { product work on } \\
\text { the user prototype } \\
\text { and to the } \\
\text { manufacture and } \\
\text { sale }\end{array}$ & $\begin{array}{l}\text { Sloan School of } \\
\text { Management, } \\
\text { MIT }\end{array}$ & USA & $\begin{array}{l}\text { Empirical paper } \\
\text { (quantitative } \\
\text { approach: } \\
\text { survey) }\end{array}$ \\
\hline
\end{tabular}

\section{Data Analysis and Findings}

Disregarding aspects such as the number of pages of the contributions and other characteristics, the selected material was finally analysed. The main findings are described below.

\subsection{Publication Trend}

Analyzing the number of contributions in the analysed sample, subdivided by year (Figure 1), it is possible to detect an exponential growth in the studies devoted to the explored subject, especially starting from 2001-2002 (RQ1). 


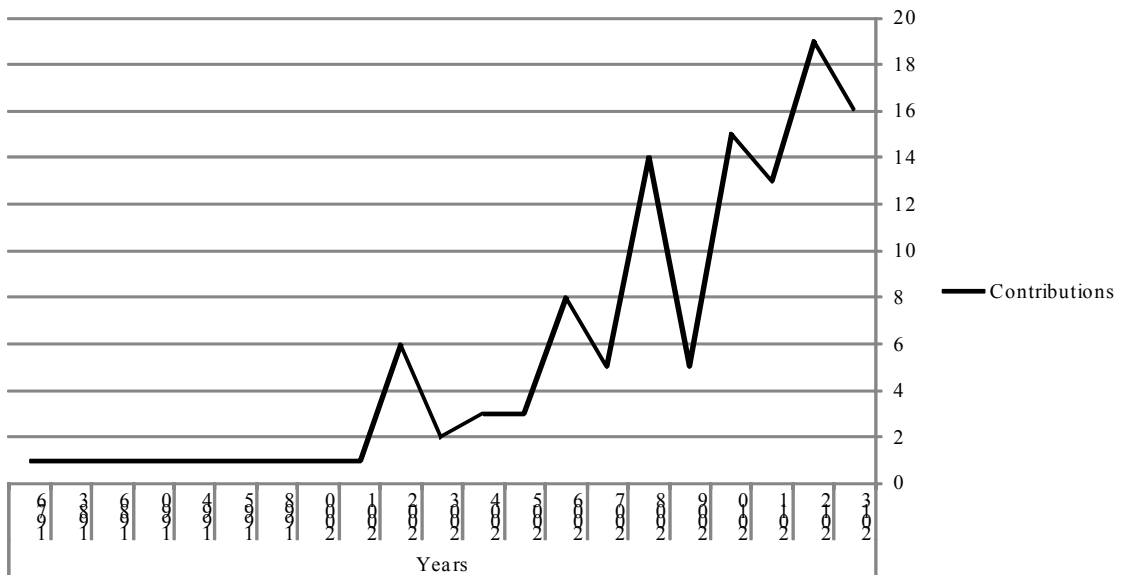

Figure 1. Publication trend

\subsection{Academic Area / Research Setting}

The highest number of contributions in the analysed sample (RQ2) have been produced in the Technology and Innovation Management (22.88\%) area. The areas which follow are (Figure 2).

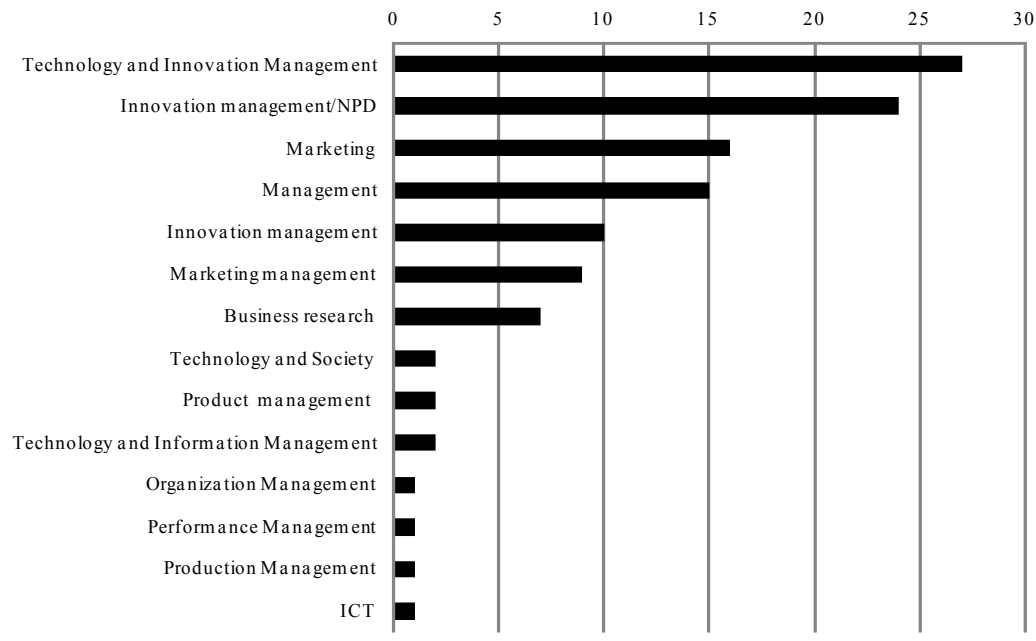

Figure 2. Academic area/research setting

NPD (20.34\%); Marketing (13.56\%);Management (12.71\%);Innovation management (8.47\%);Marketing management (7.63\%); Business research (5.93\%); Technology and Society (1.69\%), Product management (1.69\%) and Technology \& Information Management (1.69\%);Organization Management (0.85\%), Performance Management (0.85\%) and Production Management; ICT (0.85\%).

\subsection{Scientific and academic journals ranking}

By limiting the analysis to scientific journals - considering the interdisciplinary nature of the explored topic - it becomes clear that contributions are fragmented in a large number of heterogeneous sources (RQ3), which present equally variable bibliometric indicators (Figure 3). Using the Impact Factor (IF) developed by Thomson Reuters (chosen among the most widespread evaluation parameters) the relative value of all indexed periodicals in the ISItable was verified (Note 2). 


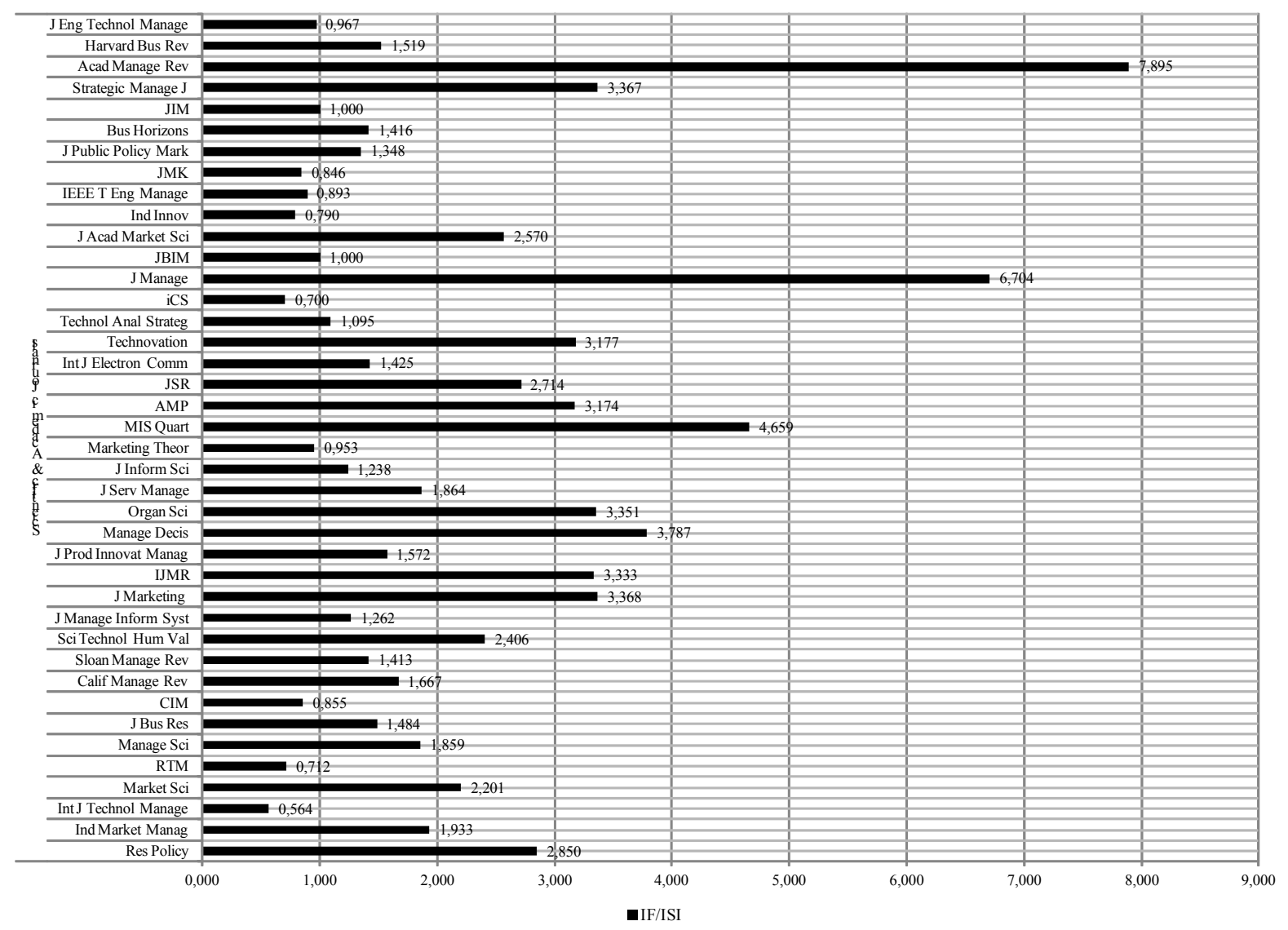

Figure 3. Journal rankings (IF/ISI)

\subsection{Represented Countries}

The most prolific country (RQ4) in terms of publication of scientific-academic contributions on the topic in the considered sample are the United States with 50\% (59) of all contributions, followed by:

Austria, 14.41\% (17); Denmark 13.56\% (16); Germany 11.86\% (14); Italy 7.63\% (9); Australia $6.78 \%(8)$ and Canada 6.78\% (8); Great Britain 5.93\% (7); The Netherlands 5.08\% (6); Finland 4.24\% (5); France 3.39\% (4) and Switzerland 3.39\% (4); China and Taiwan 2.54\% (3); Sweden 1.69\% (2); Belgium, Brazil, Korea, Japan, Israel, Luxembourg, Mexico, New Zealand, Nigeria, Norway, Spain with $0.85 \%$ (1 contribution/country).

\subsection{Types of studies}

The prevalent type of study (RQ5) are empirical contributions (Figures 5 and 6) (55\%, i.e. 65 papers), followed by theoretical-conceptual contributions (41\%, 48 papers) and methodological studies $(4 \%, 5)$.

Among empirical papers, the highest number of studies follows a qualitative approach (59\%, i.e. 38 of them), followed by papers based on qualitative methods $(26 \%$, i.e. 17) and on mixed approaches, which use a mix of quantitative and qualitative tools ( $15 \%$, i.e. 10 papers).

Among qualitative studies, the prevalent approach is that of the case study $(92.10 \%$, i.e. 35 papers), more specifically single case study $68.57 \%$ (24 papers) and multiple case study $31.43 \%$ (11 papers). 


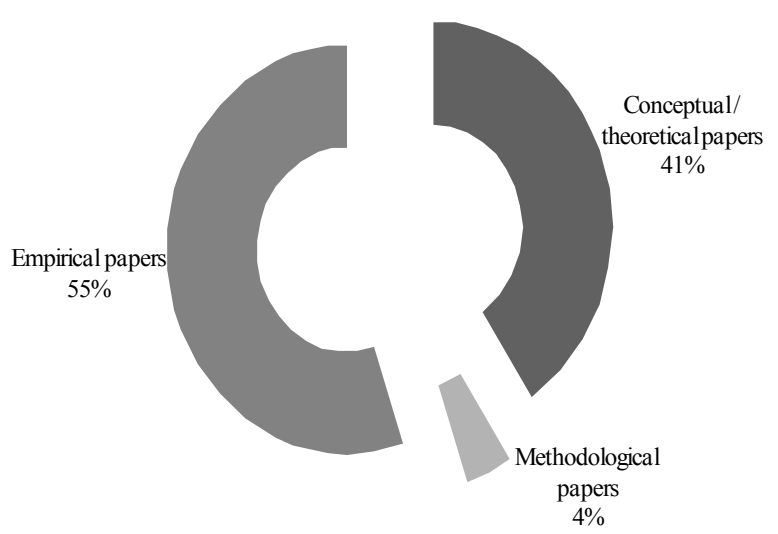

Figure 4. Types of contributions

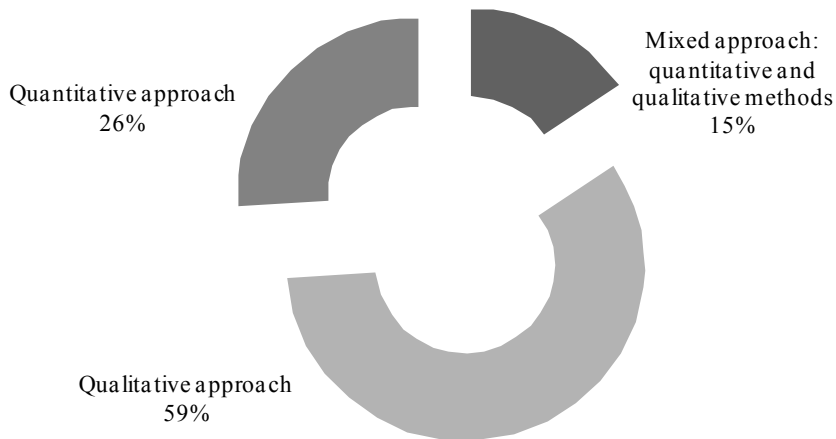

Figure 5. Empirical papers: methods

\subsection{Key Topics}

The most studied topics or sub-topics (RQ6a) in the considered sample (Figure 7) are as follows:

New role of the empowered consumer in the innovation process (19.49\%) and Consumer competence (19.49\%); Co-creation with consumers (14.41\%); Market-driven / demand pull / market oriented innovation (13.55\%); Open Innovation (OI) paradigm (11.86\%); Virtual worlds and opportunities for firms to collaborate with co-creating users (10.17\%) and Benefits of the Web for NPD user involvement (10.17\%); Online communities and innovation dynamics (7.63\%); Lead-users (6.78\%); User innovation (5.08\%) and User centric innovation (5.08\%); Crowdsourcing (3.39\%) and User toolkits for innovation - focus on instrumental features - (3.39\%); Effect of collaborative strategies - on innovation, product price, consumer - $(2.54 \%)$; User vs. producers / professionals in generating ideas and innovation (1.69\%).

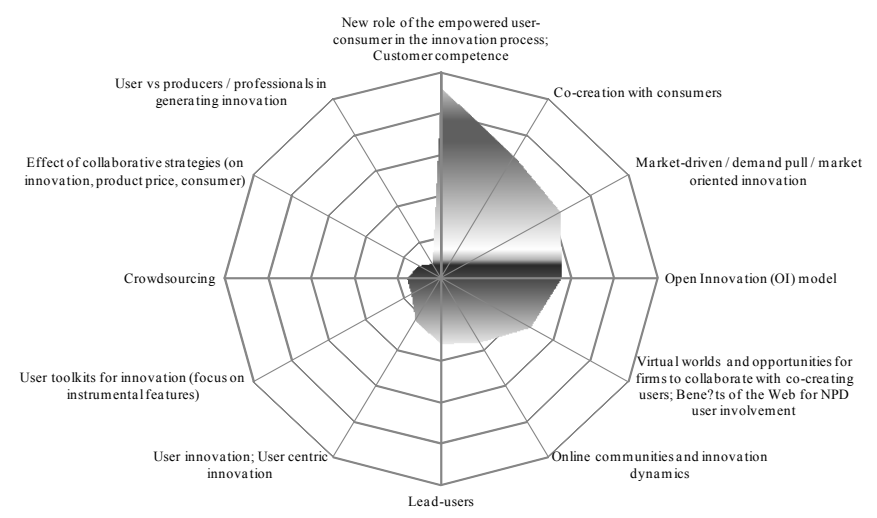

Figure 6. Key topics 


\subsection{Keyword Clusters}

The keywords supplied by the paper authors (RQ6b) - after removing the lexical elements considered as secondary in terms of relevance (Note 3) for the studied topic - were indexed in 17 homogeneous thematic groups. They were categorized by grouping occurrences with a similar content. The identified semantic clusters, in order of identified absolute frequency, are the following (Figure 8): Innovation (15.41\%); Consumer-centricity in innovation process $(11.65 \%)$; Community, online community $(10.15 \%)$; Co-creation $(8.65 \%)$ and New consumer role (8.65\%); Technology, virtual environments (8.27\%); Product development (7.14\%); Collaboration (6.77\%); Openness (6.39\%); Value (4.13\%); Idea (2.63); Network (2.25\%) and Knowledge (2.25\%); Crowdsourcing (1.88\%) and Customization (1.88\%); B2C (1.13\%) and Engagement (1.13\%).

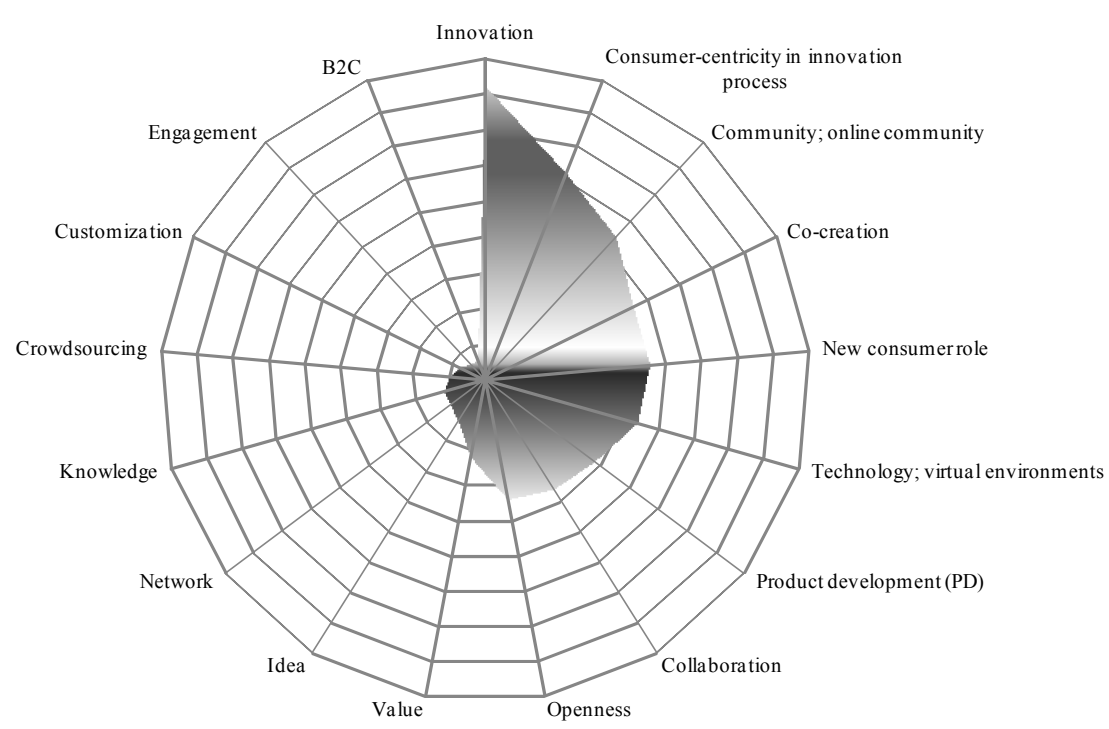

Figure 7. Keyword clusters

\subsection{Authorship}

The prevalent type of paper has multi-authorship or shared authorship ( $76.27 \%$, i.e. 90 papers), while the sole-authored type accounts for $23.73 \%$ (28) of all papers. Contributions which can be considered as having multi-institutional and multi-national authorship-namely are the result of the cooperation between several authors belonging to scientific institution in different countries-are the minority (35.59\%, i.e. 42 papers), while papers which involve researchers from the same country are $64.41 \%$ (76) of the total.

\subsection{Most Prolific Authors}

In terms of publication of scientific-academic contributions on the topic in the considered sample (Table 2) the most prolific authors are: Eric von Hippel; Johann Füller; Cornelius Herstatt; Martin Schreier; Satish Nambisan; Christoph Fuchs; Kurt Matzler; Venkat Ramaswamy.

Table 2. Most-prolific authors

\begin{tabular}{lllllllll}
\hline & \multicolumn{3}{c}{ (continued) } & \multicolumn{5}{c}{ (continued) } \\
\hline Name & No. & $\boldsymbol{\%}$ & Name & No. & \% & Name & No. & \% \\
\hline von Hippel E. & 13 & 11,02 & Devaraj S.S. & 1 & 0,85 & Mahr D. & 1 & 0,85 \\
Füller J. & 8 & 6,78 & Di Stefano G. & 1 & 0,85 & Mansour-Cole D. & 1 & 0,85 \\
Herstatt C. & 6 & 5,08 & Djelassi S. & 1 & 0,85 & Marsden J.R. & 1 & 0,85 \\
Schreier M. & 6 & 5,08 & Dorotic M. & 1 & 0,85 & Mele C. & 1 & 0,85 \\
Nambisan S. & 5 & 4,24 & Dutton W.H. & 1 & 0,85 & Molina A. & 1 & 0,85 \\
Fuchs C. & 4 & 3,39 & Eisenberg I. & 1 & 0,85 & Morgan J. & 1 & 0,85 \\
Matzler K. & 4 & 3,39 & Ellis S.C. & 1 & 0,85 & Nambisan P. & 1 & 0,85 \\
Ramaswamy V. & 4 & 3,39 & Estellés-Arolas E. & 1 & 0,85 & Narver J.C. & 1 & 0,85 \\
\hline
\end{tabular}




\begin{tabular}{|c|c|c|c|c|c|c|c|c|}
\hline Baron R.A. & 3 & 2,54 & Etgar M. & 1 & 0,85 & Nyström A.G. & 1 & 0,85 \\
\hline Berthon P.R. & 3 & 2,54 & Eze S.C. & 1 & 0,85 & Obrist M. & 1 & 0,85 \\
\hline Chesbrough $\mathrm{H}$. & 3 & 2,54 & Fang W.C. & 1 & 0,85 & Ogawa S. & 1 & 0,85 \\
\hline Piller F.T. & 3 & 2,54 & Gales L. & 1 & 0,85 & Ojanen V. & 1 & 0,85 \\
\hline Pitt L.F. & 3 & 2,54 & Gambardella A. & 1 & 0,85 & Olander H. & 1 & 0,85 \\
\hline Prahalad C.K. & 3 & 2,54 & Gan C. & 1 & 0,85 & Ollila S. & 1 & 0,85 \\
\hline Prandelli E. & 3 & 2,54 & Gandia R. & 1 & 0,85 & Olson David L. & 1 & 0,85 \\
\hline Baldwin C. & 2 & 1,69 & Garry T. & 1 & 0,85 & Parmentier G. & 1 & 0,85 \\
\hline Bogers M. & 2 & 1,69 & Gasparin M. & 1 & 0,85 & Pazgal A. & 1 & 0,85 \\
\hline Dahl D.W. & 2 & 1,69 & Gassmann $\mathrm{O}$ & 1 & 0,85 & Pezzei R. & 1 & 0,85 \\
\hline Dahlander L. & 2 & 1,69 & Gebauer J. & 1 & 0,85 & Poetz M.K. & 1 & 0,85 \\
\hline Franke N. & 2 & 1,69 & Gemuenden H.G. & 1 & 0,85 & Porter C.E. & 1 & 0,85 \\
\hline Frederiksen L. & 2 & 1,69 & Gianiodis P.T. & 1 & 0,85 & Raasch C. & 1 & 0,85 \\
\hline Frow $\mathrm{P}$. & 2 & 1,69 & Giannopoulou E. & 1 & 0,85 & Repo P. & 1 & 0,85 \\
\hline Jawecki G. & 2 & 1,69 & Godin B. & 1 & 0,85 & Romero D. & 1 & 0,85 \\
\hline Jespersen K.R. & 2 & 1,69 & Goduscheit R.C. & 1 & 0,85 & Rossi C. & 1 & 0,85 \\
\hline Kohler T. & 2 & 1,69 & González-Ladrón-de-Guevara F. & 1 & 0,85 & Rullani F. & 1 & 0,85 \\
\hline Lakhani K.R. & 2 & 1,69 & Greer C.R. & 1 & 0,85 & Russo-Spena T. & 1 & 0,85 \\
\hline Lettl C. & 2 & 1,69 & Gyrd-Jones R.I. & 1 & 0,85 & Salter A. & 1 & 0,85 \\
\hline Lichtenthaler U. & 2 & 1,69 & Hallikas J. & 1 & 0,85 & Sasinovskaya O. & 1 & 0,85 \\
\hline McCarthy I. & 2 & 1,69 & Harwood T. & 1 & 0,85 & Sawhney M. & 1 & 0,85 \\
\hline Morrison P.D. & 2 & 1,69 & Hatch MJ. & 1 & 0,85 & Schau H.J. & 1 & 0,85 \\
\hline Mühlbacher H. & 2 & 1,69 & Hauser J.R. & 1 & 0,85 & Schweitzer F.M. & 1 & 0,85 \\
\hline Payne A.F. & 2 & 1,69 & Hautz J. & 1 & 0,85 & Searls K. & 1 & 0,85 \\
\hline Schultz M. & 2 & 1,69 & Heiskanen E. & 1 & 0,85 & Secchi E. & 1 & 0,85 \\
\hline Storbacka K. & 2 & 1,69 & Hemetsberger A. & 1 & 0,85 & Sheth J.N. & 1 & 0,85 \\
\hline Verona G. & 2 & 1,69 & Hoyer W. & 1 & 0,85 & Singh S.S. & 1 & 0,85 \\
\hline von Krogh G. & 2 & 1,69 & Hutter K. & 1 & 0,85 & Slater S.F. & 1 & 0,85 \\
\hline West J. & 2 & 1,69 & Hyysalo S. & 1 & 0,85 & Sonnack M. & 1 & 0,85 \\
\hline Afuah A. & 1 & 0,85 & Iglesias O. & 1 & 0,85 & Spaeth S. & 1 & 0,85 \\
\hline Alexy O. & 1 & 0,85 & Ind $N$. & 1 & 0,85 & Stieger D. & 1 & 0,85 \\
\hline Anderson $\mathrm{H}$. & 1 & 0,85 & Jensen M.B. & 1 & 0,85 & Stuermer M. & 1 & 0,85 \\
\hline Awa H.O. & 1 & 0,85 & Jeppesen L.B. & 1 & 0,85 & Sun D. & 1 & 0,85 \\
\hline Bae Z. & 1 & 0,85 & Jørgensen J.H. & 1 & 0,85 & Swink M. & 1 & 0,85 \\
\hline Bastian B. & 1 & 0,85 & Kang S.H. & 1 & 0,85 & Syam N.B. & 1 & 0,85 \\
\hline Bayus B.L. & 1 & 0,85 & Kates S.M. & 1 & 0,85 & Thomke S. & 1 & 0,85 \\
\hline Bilgram V. & 1 & 0,85 & Katz R. & 1 & 0,85 & Tietz R. & 1 & 0,85 \\
\hline Bin Guo & 1 & 0,85 & Keinz P. & 1 & 0,85 & Trimi S. & 1 & 0,85 \\
\hline Blomqvist K. & 1 & 0,85 & Kim J.H. & 1 & 0,85 & Uncles M.D. & 1 & 0,85 \\
\hline Borella Guido A.L. & 1 & 0,85 & King A. & 1 & 0,85 & Uslay C. & 1 & 0,85 \\
\hline Brabham D.C. & 1 & 0,85 & Kornum N. & 1 & 0,85 & Vargo S.L. & 1 & 0,85 \\
\hline Braga A.C. & 1 & 0,85 & Kosonen M. & 1 & 0,85 & Varnes C.J. & 1 & 0,85 \\
\hline Brem A. & 1 & 0,85 & Kotro T. & 1 & 0,85 & Voigt K.-I. & 1 & 0,85 \\
\hline Brockhoff K. & 1 & 0,85 & Kozinets R.V. & 1 & 0,85 & Walcher D. & 1 & 0,85 \\
\hline Buchinger W. & 1 & 0,85 & Krafft M. & 1 & 0,85 & Wang R & 1 & 0,85 \\
\hline Campbell C.L. & 1 & 0,85 & Lane J.P. & 1 & 0,85 & Warnke P. & 1 & 0,85 \\
\hline Chandy R. & 1 & 0,85 & Las Casas A.L. & 1 & 0,85 & Watson R.T. & 1 & 0,85 \\
\hline Chen L. & 1 & 0,85 & Lee S.M. & 1 & 0,85 & Weber M. & 1 & 0,85 \\
\hline Christiansen J.K. & 1 & 0,85 & Lei D. & 1 & 0,85 & Westerlund M. & 1 & 0,85 \\
\hline Cova B. & 1 & 0,85 & Leitner K.H. & 1 & 0,85 & Wu S.C. & 1 & 0,85 \\
\hline Criscuolo P. & 1 & 0,85 & Leminen S. & 1 & 0,85 & Wynn D. & 1 & 0,85 \\
\hline Dahan E. & 1 & 0,85 & Lievens A. & 1 & 0,85 & Yström A. & 1 & 0,85 \\
\hline Dalli D. & 1 & 0,85 & Lilien G.L. & 1 & 0,85 & Zhang Z. & 1 & 0,85 \\
\hline Danneels E. & 1 & 0,85 & Lock P. & 1 & 0,85 & Zinkhan G. & 1 & 0,85 \\
\hline Day G.S. & 1 & 0,85 & Luthje C. & 1 & 0,85 & Zwass V. & 1 & 0,85 \\
\hline Decoopman I. & 1 & 0,85 & Macdonald E.K & 1 & 0,85 & Zwick D. & 1 & 0,85 \\
\hline
\end{tabular}




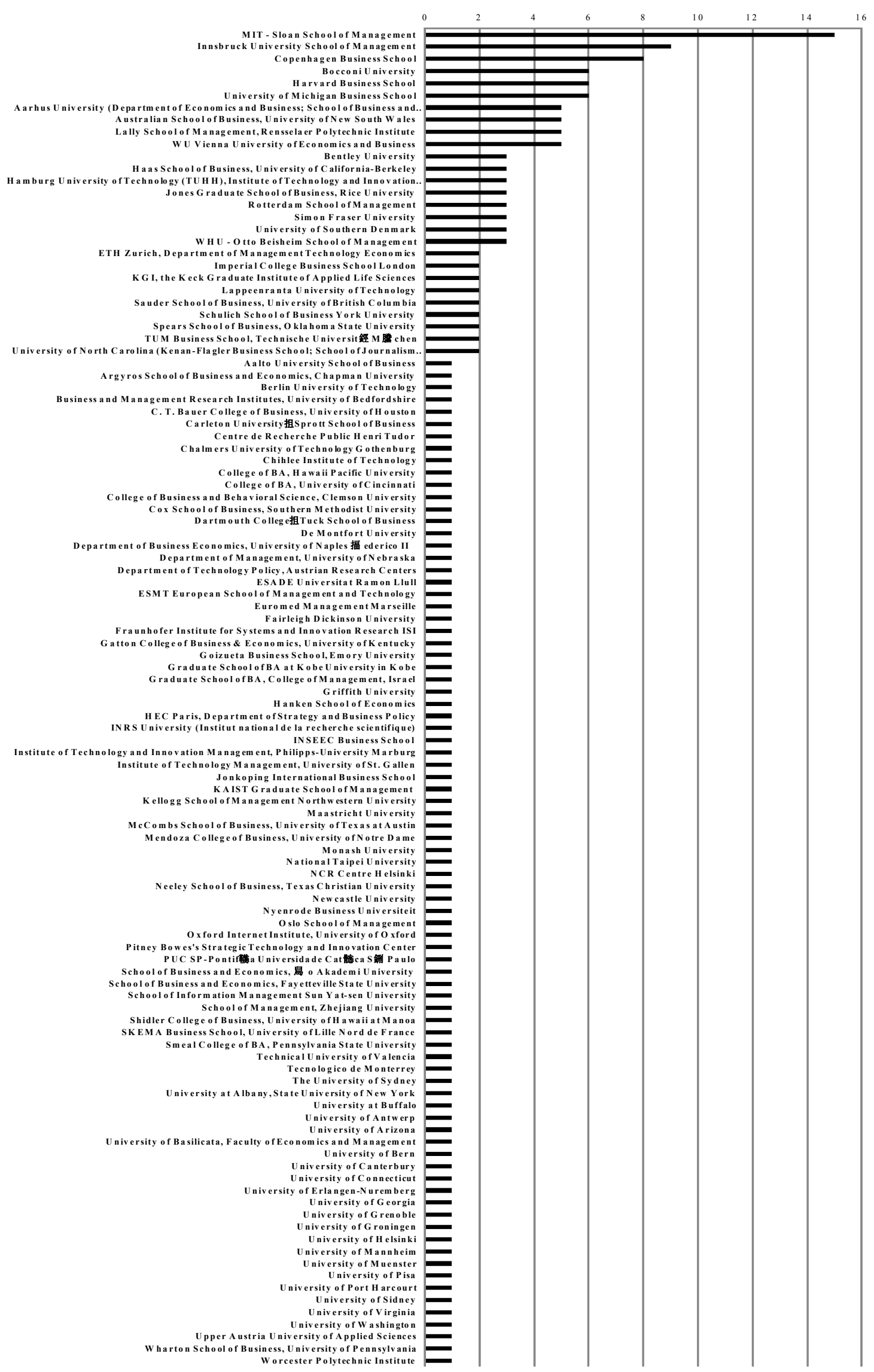

Figure 8. Represented scientific institutions 


\subsection{Scientific Institutions (Academic Organisations, Research Centres)}

The most prolific scientific institution (RQ7) in terms of publication of scientific-academic contributions on the topic in the considered sample (Figure 9) is the MIT Sloan School of Management, the business school of the Massachusetts Institute of Technology, in Cambridge, Massachusetts, USA.

Three European institutions follow: the business school of the Austrian Innsbruck University, the Danish Copenhagen Business School and the Italian Bocconi University.

\section{Conclusion}

\subsection{Discussion}

This article presents an overview of the state of the art in the research on consumer involvement in marketing and innovation processes. Different aspects stand out in this review. The field of study is still relatively young and thus attracts many researchers from different study domains. They bring different approaches, constructs, and methods to their analyses, which explain why similar goals are assessed in different ways. Interdisciplinary influences are expected to continue to contribute to more theory building. A wealth of methods means a wealth of findings, perspectives, and details, but, at the same time, heterogeneity leads to difficulties in comparing results among studies.

Specifically, when it comes to the specific research questions to which the study aims to answer, the analysis first shows a remarkable growth in the publishing activity in this area in recent years, which is expected to continue to flourish. As expected, a high fragmentation of the scientific production in a high number of heterogeneous sources has also been observed. However, the highest number of studies in the sample has been produced in the Technology and Innovation Management area, which would suggest that the sudden development of digital technologies was a propelling factor, given the availability of new information and communication infrastructures to support innovation. The analysis also seems to suggest a general geographical pattern in the study of the topic, and more specifically, a"westernization". Indeed, the most prolific authors with the highest number of occurrences have an affiliation with scientific institutions in North America or Europe. Based on this review, the majority of studies are of empirical nature. As a consequence, a methodological standardization in studying and analyzing the topic exists. Qualitative methods are the most widely used, especially the single case study: one can conclude that a substantial amount of published survey research is not chiefly concerned with generalisability. Finally, findings highlight that scholars have paid particular attention to the new role of the empowered consumer and to consumers' competence in the innovation process. Researchers seem to have focused their attention on the external environment as a possible source of knowledge and insights, and on its integration within firm boundaries.

\subsection{Theoretical Implications}

In line with expectations, and based on the results of this review, it can be argued that no dominant paradigm per se has emerged. Different theoretical bodies have been employed: relationship marketing studies; new product innovation (NPI) research and collaborative marketing patterns; management and organisational studies on network-firms and Open Innovation (OI).

The concept of relationship marketing (Christopher, Payne, \& Ballantyne, 1991; McKenna, 1991; Pepper \& Rogers, 1993) and the research streams derived from it, apply the metaphor of personal relationship to the exchange processes between a company and consumers. A key idea in the later development of this approach, or New Relationship Marketing (Gummesson, 2002), is the potential that the Internet has to enhance the ability of firms to engage customers in several ways, allowing companies to transform episodic and one-way interactions into a persistent dialogue with them. According to this perspective, "technological forces are shaping the practice of Relationship Marketing" (Sheth, Parvatiyar, \& Sinha, 2012, p. 10) and without an effective use of technology, relationship marketing is not an effective strategy (Zineldin, 2000). In embracing the key assumption that relationships with customers play a central role in the process of economic value creation, the evolution of this idea into a tool in Customer Relationship Management (CRM) also offers a strategic connection between marketing strategies and ICTs (Jayachandran, Sharma, Kaufman, \& Raman, 2005), thus creating the paradigm of information-enabled relationship marketing (Ryals \& Payne, 2001). Ahuja and Medury (2010) follow the same approach: it becomes essential to create and maintain adequate Customer Information Management systems: "companies interact with customers, treat them as organizational assets, learn about them and through the process of incorporating feedback and co-creation" (ivi, p. 94).

While collaboration with customers can involve several business processes, one of the most important aspects is cooperating to create value through product innovation. NPI research and the related international empirical 
literature have given great impetus to co-creation mechanisms (Von Hippel, 1976, 1978, 1986, 1988; Day, 1991; Webster, 1994; Gales \& Mansour-Cole, 1995; Bruce, Leverick, Littler, \& Wilson, 1995; Slater \& Narver, 1998; Verona, 1999; Thomke, 1998, 2002; Danneels, 2002; Urban \& Hauser, 2002), acknowledging the power of collaboration and going beyond a company's borders and demand-pull dynamics as the main factors behind this process in current times.

Literature on collaborative marketing (Prahalad \& Ramaswamy, 2000, 2004a, 2004b, 2004c; Vargo \& Lusch, 2004; 2006; 2008; Payne, Storbacka, \& Frow, 2008) presents the concept of convergence between consumers and producers as an invitation to combine their respective skills in a partnership. The main focus is the role of companies in identifying and supporting those consumers' activities which lead to value creation.

The contribution of management studies on OI (Chesbrough, 2003, 2006, 2011; Nambisan \& Sawhney, 2007) and on the network and systemic model of organisations (Butera, 1995; Senge, Kleiner, Roberts, Ross, Roth, \&Smith, 1994; Lane, Serra, Villani, \& Ansaloni, 2006; Golinelli, 2010) is particularly relevant in connection to the idea of a collective enterprise. This is linked to the disappearance of organisational borders, the emergence of looser relationships and the enlargement of a company's network. In a systemic approach, knowledge (Nonaka \& Takeuchi, 1996) and skills owned by consumers are a great potential available to companies to foster their evolution processes.

The combination of these separate theoretical bodies allows the identification of key concepts which, extrapolated from the reviewed literature, try to grasp the main dimensions that have to be taken into account to explore the complex nature of the investigated topic: relationship, consumer engagement, value co-creation, openness. Each research stream, within its discipline of reference, takes on a different epistemological perspective, which highlights complementary aspects.

\subsection{Limitations and Directions for Future Research}

This study presents a recent review of the topic, but some limitations of the analysis carried out should also be highlighted. First, findings are limited by the fast-paced nature of the publishing activity. Second, the analysis carried out, although allowing work on a robust database, focuses on journal articles, conference and work papers and selects contributions on the basis of keywords. It would be useful to extend the analysis to scholarly handbooks, book chapters and monographs. A network analysis linking scholars and thematic content areas would also be interesting. Third, the author recognizes that the review is mainly carried out from a corporate perspective, without taking into account consumer responses to collaborative dynamics. Future reviews may further analyze the topic going beyond the managerial point of view, extending the review by including other topics linked to the micro-level where consumers act. This could allow the identification of possible contributions related to the effectiveness of collaborative approaches in relation to different consumer audiences and their responses (for example, the relationship between the topic and firm/brand reputation, consumer loyalty, effectiveness of products in terms of greater closeness to customer needs, positioning in terms of better differentiation). Some methodological issues also emerged after examining the contributions surveyed in this review. In particular, it is deemed important to rely more on methods that today appear to be under-utilized in order to enrich the field with heterogeneous perspectives: methodological studies which suggest new methods and systems to analyse and measure the phenomenon; empirical papers of quantitative-extensive type and with a longitudinal design, which would allow causality inference and generalisability; theoretical contributions attempting to link and integrate definitions, concepts and insights originating from different research settings, placing them within holistic interpretative frameworks with practical and managerial implications to deal with the collaborative process.

Future research should also be conducted on costs, risks and outcomes related to the implementation of collaborative processes between companies and consumers and on the development of metrics to measure them. The quality and quantity of external contributions-of potentially unlimited quantity and of highly variable quality - may create overwork in terms of time management, skills and planning needed to deal with this flow and mutual exchange, as well as opportunities for managers themselves. In such situations, problems about scarcity of resources and attention become particularly important. Finally, adopting an interdisciplinary, multi-functional approach, future research should also look at the following other aspects:

- Collaborative processes and specific company's features;

- Types of external contributors and different stages of collaborative processes: which consumer segments should firms target at each stage?

- $\quad$ Firm-level and consumer-level impediments of co-creation; 
- Collaborative dynamics, company's boundaries extension, corporate identity: is a hyper-adaptation risk feasible?

- Perspectives for the Organizational Communication discipline: support for the creation of an open mentality to tackle the 'not invented here' syndrome; managing possible tensions linked to the impact of co-creation processes which involve external subjects on the company's micro-culture - the consumer owns competences and skills and is placed in the condition of turning them into practice, however it is possible that the internal resources could report a reduction of power;

- Perspectives for the PR field: strategic support in the management of external collaborative processes with the aim of limiting possible critical points;

- Relationship between collaborative approaches and the evolution of the concept of Corporate Social Responsibility (CSR), moving towards the "umbrella" definition of Sustainability, which includes the traditional elements of green/environmental policy, as well as new aspects related to the concept of stakeholder engagement, economic and business behaviour, respect of privacy and, more importantly, the inclusion of the 'consumerism' component related to the preferences and desires of consumer segments;

- Relationship between the communication of the open innovation strategy engaging consumers and the macro industrial company's paradigm (please refer to: Rindova \& Fombrun, 1999): does it have any influence on the evolution of the served market dynamics?

\section{References}

Adomavicius, G., \& Tuzhilin, A. (2005). Toward the Next Generation of Recommender Systems: A Survey of the State-of-the-Art and Possible Extensions. EEE Transactions on Knowledge and Data Engineering, 17(6), 734-749. http://dx.doi.org/10.1109/TKDE.2005.99

Afuah, A. (2003). Redefining Firm Boundaries in the Face of the Internet: Are Firms Really Shrinking? Academy of Management Review, 28(1), 34-53. http://dx.doi.org/10.5465/AMR.2003.8925207

Ahuja, V., \& Medury, Y. (2010). Corporate blogs as e-CRM tools-Building consumer engagement through content management. Journal of Database Marketing and Customer Strategy Management, 17(2), 91-105. http://dx.doi.org/10.1057/dbm.2010.8

Berthon, P. R., Campbell, C. L., Pitt, L. F., \& McCarthy, I. (2011). Creative Consumers: Awareness, Attitude and Action. Journal of Consumer Marketing, 28(7), 500-507.http://dx.doi.org/10.1108/07363761111181482

Berthon, P. R., Pitt, L. F., McCarthy, I., \& Kates, S. M. (2007). When Customers Get Clever: Managerial Approaches to Dealing with Creative Consumers. Business Horizons, 50(1), 39-48. http://dx.doi.org/10.1016/j.bushor.2006.05.005

Bilgram, V., Brem, A., \& Voigt, K. I. (2008). User-Centric Innovations in New Product Development-Systematic Identification of Lead Users Harnessing Interactive and Collaborative Online-Tools. International Journal of Innovation Management, 12(3), 419-458. http://dx.doi.org/10.1142/S1363919608002096

Bruce, M, Leverick, F., Littler, D., \& Wilson D. (1995). Success factors for collaborative product development: a study of suppliers of information and communication technology. $R \& D$ Management, 25(1), 33-44. http://dx.doi.org/10.1111/j.1467-9310.1995.tb00898.x

Butera, F. (1995). Human oriented management of Change. A conceptual model. Advances in Human Factors/Ergonomics, 20/b, 1003-1010. http://dx.doi.org/10.1016/S0921-2647(06)80344-8

Chen, Y., \& Xie, J. (2008). Online Consumer Review: Word-of-mouth as A New Element of Marketing Communication Mix. Management Science, 54(3), 477-491. http://dx.doi.org/10.1287/mnsc.1070.0810

Chesbrough, H. (2003). Open Platform Innovation: Creating Value from Internal and External Innovation. Intel Technology Journal, $7(3), \quad 5-9$. https://noggin.intel.com/content/open-platform-innovation-creating-value-from-internal-and-external-innov ation

Chesbrough, H. (2006). Open Business Models: How to Thrive in the New Innovation Landscape. Boston: Harvard Business School Press.

Chesbrough, H. (2011). Open Services Innovation: Rethinking Your Business to Grow and Compete in a New Era. San Francisco: Jossey-Bass. A Wiley Imprint.

Choi, S. H., \& Cheung, H. H. (2008). A versatile virtual prototyping system for rapid product development. 
Computers in Industry, 59, 477-488. http://dx.doi.org/10.1016/j.compind.2007.12.003

Christopher, M., Payne, A., \& Ballantyne, D. (1991). Relationship Marketing. Oxford: Butterworth-Heinemann.

Cova, B., \& Cova, V. (2012). On the road to prosumption: marketing discourse and the development of consumer competencies. Consumption Markets and Culture, 15(2), 149-168. http://dx.doi.org/10.1080/10253866.2012.654956

Cova, B., \& Dalli, D. (2009). Working Consumers: The Next Step in Marketing Theory? Marketing Theory, 9(3), 315-339. http://dx.doi.org/10.1177/1470593109338144

Cova, B., \&White, T. (2010). Counter-brand and alter-brand communities: the impact of Web 2.0 on tribal marketing approaches. Journal of Marketing Management, 26(3/4), 256-270. http://dx.doi.org/10.1080/02672570903566276

Cova, B., Dalli, D., \& Zwick, D. (2011). Critical perspectives on consumers' role as 'producers': Broadening the debate on value co-creation in marketing processes. Marketing Theory, 11(3), 231-241. http://dx.doi.org/10.1177/1470593111408171

Cova, B., Kozinets, R. V., \& Shankar, A. (2007). Consumer Tribes. Oxford: Elsevier Butterworth Heinemann.

Dahan, E., \& Hauser, J.R. (2002). The Virtual Customer. Journal of Product Innovation Management, 19(5), 332-354. http://dx.doi.org/10.1111/1540-5885.1950332

Danneels, E. (2002). The Dynamics of Product Innovation and Firm Competences. Strategic Management Journal, 23(12), 1095-1121. http://dx.doi.org/10.1002/smj.275

Day, G. (1991). Learning about markets Marketing Association Report (pp. 91-117). Cambridge (MA): Marketing Science Institute.

Di Stefano, G., Gambardella, A., \& Verona, G. (2012). Technology push and demand pull perspectives in innovation studies: Current findings and future research directions. Research Policy, 41(8), 1283-1295. http://dx.doi.org/10.1016/j.respol.2012.03.021

Echeverri, P., \& Skålen P. (2011). Co-creation and co-destruction: a practice theory based study of interactive value formation. Marketing Theory, 11(3), 351-373. http://dx.doi.org/10.1177/1470593111408181

Fisher, D., \& Smith, S. (2011). Co-creation is chaotic: What it means for marketing when no one has control. Marketing Theory, 11, 325-350. http://dx.doi.org/10.1177/1470593111408179

Ford, D. (2004). The IMP Group and International Marketing. International Marketing Review, 21(2), 139-141. http://dx.doi.org/10.1108/02651330410531358

Frow, P., Payne, A. F., \& Storbacka, K. (2011). Co-Creation: A Typology and Conceptual Framework. In Proceedings of ANZMAC 2011, Perth, WA, 1-6. http://anzmac.info/conference/2011/Papers\%20by\%20Track/Track\%2011.\%20Relationship\%20Marketing/ Payne,\%20Adrian\%20Paper\%20366.pdf

Füller, J., \& von Hippel E. (2008). Costless Creation of Strong Brands by User Communities: Implications for Producer-Owned Brands. MIT Sloan School of Management Working Paper, 1-30. http://dx.doi.org/10.2139/ssrn.1275838

Füller, J., Muhlbacher, H., Matzler, K., \& Jawecki G. (2009). Consumer empowerment through internet-based co-creation. Journal of Management Information Systems, 26, 71-102. http://dx.doi.org/10.2753/MIS0742-1222260303

Gales, L., \& Mansour-Cole D. (1995). User involvement in innovation projects: Toward a theory of user information processing. Journal of Engineering and Technology Management, 12, 77-109. http://dx.doi.org/10.1016/0923-4748(95)00005-7

Gianiodis, P. T., Ellis, S. C., \& Secchi, E. (2010). Advancing a typology of open innovation strategies. International Journal of Innovation Management, 14(4), 531-572. http://dx.doi.org/10.1142/S1363919610002775

Giannopoulou, E., Yström, A., \& Ollila, S. (2011). Turning open innovation into practice: open innovation research through the lens of managers. International Journal of Innovation Management, 15(3), 505-524. http://dx.doi.org/10.1142/S1363919611003465

Golinelli, G. M. (2010). Viable Systems Approach (VSA). Governing Business Dynamic. Padova, IT: Cedam, Kluwer. 
Greer, C. R., \& Lei, D. (2012). Collaborative Innovation with Customers: A Review of the Literature and Suggestions for Future Research. International Journal of Management Reviews, 14, 63-84. http://dx.doi.org/10.1111/j.1468-2370.2011.00310.x

Grönross, C. (1990). Relationship Approach to Marketing in Service Contexts: The Marketing and Organizational Behavior Interface. Journal of Business Research, 20(1), 3-11. http://dx.doi.org/10.1016/0148-2963(90)90037-E

Gummesson, E., \& Polese, F. (2009). B2B is not an island! Journal of Business and Industrial Marketing, 24(5/6), 337-350. http://dx.doi.org/10.1108/08858620910966228

Gummesson, E. (2002). Relationship marketing in the new economy. Journal of Relationship Marketing, 1(1), 37-57. http://dx.doi.org/10.1300/J366v01n01_04.

Håkansson, H., \& Lundgren, A. (1995). Industrial Networks and Technical Innovation. In Möller K., \& Wilson D. Boston (Eds.), Business Marketing: An Interaction and Network Perspective. USA: Kluwer Academic Publishers.

Håkansson, H. (1982). International Marketing and Purchasing of Industrial Goods - An Interaction Approach. Chichester: IMP Project Group, John Wiley and Sons.

Håkansson, H., Ford, D., Gadde, L-E., Snehota, I., \& Waluszewski A. (2009). Business in Networks. Chichester, England: John Wiley and Sons Ltd.

Hoyer, W. D., Chandy, R., Dorotic, M., Krafft, M., \& Singh, S. S. (2010). Consumer Co-creation in New Product Development. Journal of Service Research, 13(3), 283-296. http://dx.doi.org/10.1177/1094670510375604

Iglesias, O., Ind, N., \& Alfaro, M. (2013). The organic view of the brand: A brand value co-creation model. The Journal of Brand Management, 20(8), 670-688. http://dx.doi.org/10.1057/bm.2013.8

Jayachandran, S., Sharma, S., Kaufman, P., \& Raman, P. (2005). The role of relational information processes and technology use in customer relationship management. Journal of Marketing, 69(4) 177-192. http://dx.doi.org/10.1509/jmkg.2005.69.4.177

Jespersen, K. R. (2011). Online Channels and Innovation: Are Users being Empowered and Involved? International Journal of Innovation Management, 15(6), 1141-1159. http://dx.doi.org/10.1142/S1363919611003684

Kärkkäinen, H., Jussila, J., \& Väisänen, J. (2013). Social Media Use and Potential in Business-to-Business Companies' Innovation. International Journal of Ambient Computing and Intelligence (IJACI), 5(1), 53-71. http://dx.doi.org/10.4018/jaci.2013010104.

Kassarjian, H. H. (1977). Content analysis in consumer research. Journal of Consumer Research, 4, 8-18. http://dx.doi.org/10.1086/208674

Krippendorff, K. (1980). Content Analysis: An Introduction to its Methodology. London: Sage.

Lane, D. A., Serra, R., Villani, M., \& Ansaloni, L. (2006). A Theory-Based Dynamical Model of Innovation Processes. ComPlexUs, 2, 177-194. http://dx.doi.org/10.1159/000093689

McKenna, R. (1991). Relationship Marketing: Successful Strategies for the Age of the Customer. Cambridge, MA: Perseus Publishing.

Morgan, J., \& Wang, R. (2010). Tournament for Ideas. California Management Review, 52(2), 77-97. http://dx.doi.org/10.1525/cmr.2010.52.2.77

Nambisan, S., \& Sawhney, M. (2007). The global brain: Your Roadmap for Innovating Faster and Smarter in a Networked World. Wharton School Publishing, PA.

Nonaka, I., \& Takeuchi, H. (1996). The knowledge-creating company: How Japanese companies create the $\begin{array}{lllll}\text { dynamics of innovation. Long Range Planning, } & \text { 29(4), }\end{array}$ http://dx.doi.org/10.1016/0024-6301(96)81509-3

Ofek, E., \& Toubia, O. (2010). Marketing and Innovation Management: An Integrated Perspective. Now Publishers Inc.

Page, K. L., \& Pitt, L. (2011). Web 2.0, Social Media, and Creative Consumers: Special Issue. Journal of Consumer Behaviour, 10(6), 313-372. http://dx.doi.org/10.1002/cb.390

Payne, A. F., Storbacka, K., \& Frow, P. (2008). Managing the co-creation of value. Journal of the Academy of 
Marketing Science, 36(1), 83-96. http://dx.doi.org/10.1007/s11747-007-0070-0

Pepper, D., \& Rogers, M. (1993). The One-to-One Future: Building Relationships One Customer at a Time. New York, NY: Currency/Doubleday.

Prahalad, C. K., \& Ramaswamy, V. (2000). Co-opting customer competence. Harvard Business Review, 78(1), $79-87$.

https://hbr.org/download/co-opting-customer-competence/R00108-PDF-ENG/R00108-PDF-ENG.PDF

Prahalad, C. K., \& Ramaswamy, V. (2004a). Co-creation Experiences: The Next Practice in Value Creation. Journal of Interactive Marketing, 18(3), 5-14. http://dx.doi.org/10.1002/dir.20015

Prahalad, C. K., \& Ramaswamy, V. (2004b). Co-creating unique value with the customers. Strategy and Leadership, 32(3), 4-9. http://dx.doi.org/10.1108/10878570410699249

Prahalad, C. K., \& Ramaswamy, V. (2004c).The Future of Competition: Co-creating Unique Value with Customers. Boston, MA: Harvard Business School Press.

Prandelli, E., Sawhney, M., \& Verona, G. (2008). Collaborating with Customers to Innovate. Conceiving and Marketing Products in the Network Age. Edgar, Sheffield Brightman.

Prandelli, E.,Verona, G., \& Raccagni, D. (2006). Diffusion of Web-based product innovation. California Management Review, 48(4), 109-135. http://dx.doi.org/10.2307/41166363.

Rindova, V. P., \& Fombrun, C. J. (1999). Constructing competitive advantage: the role of firm-constituent interactions. Strategic Management Journal, 20(8), 691-710. http://dx.doi.org/10.1002/(SICI)1097-0266(199908)20:8\%3C691::AID-SMJ48\%3E3.0.CO;2-1

Romero, D., \& Molina, A. (2011). Collaborative networked organisations and customer communities: value co-creation and co-innovation in the networking era. Production Planning and Control: The Management of Operations, 22(5-6), 447-472. http://dx.doi.org/10.1080/09537287.2010.536619

Rumrill, P. D., Fitzgerald, S. M., \& Merchant, W. R. (2010). Using scoping literature reviews as a means of understanding and interpreting existing literature. Work: A Journal of Prevention, Assessment and Rehabilitation, 35(3), 399-404. http://dx.doi.org/10.3233/WOR-2010-0998

Ryals, L., \& Payne, A. (2001). Customer relationship management in financial services: towards information-enabled relationship marketing. Journal of Strategic Marketing, 9(1), 3-27. http://dx.doi.org/10.1080/713775725

Sawhney, M., Verona, G., \& Prandelli, E. (2005). Collaborating to Create: The Internet as a Platform for Customer Engagement in Product Innovation. Journal of Interactive Marketing, 19(4), 4-17. http://dx.doi.org/10.1002/dir.20046

Senge, P. M., Kleiner, A., Roberts, R., Ross, R., Roth, G., \& Smith, B. (1994). The Fifth Discipline Fieldbook: Strategies and Tools for Building a Learning Organization. New York: Doubleday.

Sheth, J. N., \& Uslay, C. (2007). Implications of the Revised Definition of Marketing: From Exchange to Value Creation. Journal of Public Policy and Marketing, 26(2), 302-307. http://dx.doi.org/10.1509/jppm.26.2.302

Sheth, J. N., Parvatiyar, A., \& Sinha, M. (2012). The Conceptual Foundations of Relationship Marketing: Review and Synthesis. Economic Sociology-The European Electronic Newsletter, 13(3). Retrieved from http://pubman.mpdl.mpg.de/pubman/item/escidoc:1662344:1/component/escidoc:1662342/mpifg_zs12_40. pdf\#page $=4$

Slater, S. F., \& Narver, J. C. (1998). Customer-led and market-oriented: let's not confuse the two. Strategic Management Journal, 19(10), 1001-1006. http://dx.doi.org/10.1002/(SICI)1097-0266(199810)19:10\%3C1001::AID-SMJ996\%3E3.0.CO;2-4

Thomke, S., \& Von Hippel, E. (2002). Customers as Innovators: A New Way to Create Value. Harvard Business Review, 80(4), 74-81. http://hbr.org/2002/04/customers-as-innovators-a-new-way-to-create-value/ar/1

Thomke, S. (1998). Managing Experimentation in the Design of New Products. Management Science, 44(6), 743-762. http://dx.doi.org/10.1287/mnsc.44.6.743

Urban, G. L., \& Hauser, J. R. (2002). Listening in to find unmet customer needs and solutions. Working Paper, 156, July, Center for eBusiness, MIT Press. http://dx.doi.org/10.2139/ssrn.373061

Vallaster, C., \& Von Wallpach, S. (2013). An online discursive inquiry into the social dynamics of multi-stakeholder brand meaning co-creation. Journal of Business Research, Special Issue on Virtual 
Dialogue, 66(9), 1505-1515. http://dx.doi.org/10.1016/j.jbusres.2012.09.012

Vargo, S., \& Lusch, R.F. (2004). Evolving to a New Dominant Logic for Marketing. Journal of Marketing, 68, 1-17. http://dx.doi.org/10.1509/jmkg.68.1.1.24036

Vargo, S., \& Lusch, R. F. (2006). The Service Dominant Logic of Marketing. Dialog, Debate and Directions. Armonk, New York: M.E. Sharpe.

Vargo, S., \& Lusch, R. F. (2008). Service-dominant logic: continuing the evolution. Journal of the Academic Marketing Science, 36, 1-10. http://dx.doi.org/10.1007/s11747-007-0069-6

Verona, G. (1999). A Resource-based View of Product Development. The Academy of Management Review, 24(1), 132-142. http://dx.doi.org/10.5465/AMR.1999.1580445

Von Hippel, E. (1976). The Dominant Role of Users in the Scientific Instrument Innovation Process. Research Policy, 5(3), 212-239. http://dx.doi.org/10.1016/0048-7333(76)90028-7

Von Hippel, E. (1978). Successful Industrial Products From Customer Ideas. Journal of Marketing, 42, 39-49. http://dx.doi.org/10.2307/1250327

Von Hippel, E. (1986). Lead Users: A Source of Novel Product Concepts. Management Science, 32(7), 791-805. http://dx.doi.org/10.1287/mnsc.32.7.791

Von Hippel, E. (1988). The Sources of Innovation. Oxford: Oxford University Press.

Von Hippel, E. (2001). Perspective: User toolkits for innovation. Journal of Product Innovation Management, 18(4), 247-257. http://dx.doi.org/10.1016/S0737-6782(01)00090-X

Von Hippel, E., \& Katz, R. (2002). Shifting innovation to users via toolkits. Management Science, 48(7), 821-833. http://dx.doi.org/10.1287/mnsc.48.7.821.2817

Webster Jr, F. E. (1994). Market-Driven Management. New York: John Wiley and Sons.

Wind, J., \& Rangaswamy, A. (2001). Customerization: The Next Revolution in Mass Customization. Journal of Interactive Marketing, 15(1), 13-32. http://dx.doi.org/10.1002/1520-6653(200124)15:1\%3C13::AID-DIR1001\%3E3.0.CO;2-\%23

Wipperfürth, A. (2005). Brand Hijack: Marketing without Marketing. New York: Portfolio.

Zineldin, M. (2000). Beyond relationship marketing: technologicalship marketing. Marketing Intelligence and Planning, 18(1), 9-23. http://dx.doi.org/10.1108/02634500010308549

Zwick, D., Bonsu, S. K., \& Darmody, A. (2008). Putting Consumers to Work: Co-creation and new marketing govern-mentality. Journal of Consumer Culture, 8(2), 163-196. http://dx.doi.org/10.1177/1469540508090089

\section{Notes}

Note 1. The expressions user, consumer, customer, shall be used as synonyms, as the objectives of the stud do not include the identification of the role of the different types of involved figures (buyer, end user, influencer and so on). Also, by limiting the analysis to the context of $\mathrm{B} 2 \mathrm{C}$ markets, the term customer does not seem to create any confusion with the concepts of intermediate/trade customer or client meaning a purchasing organisation, as found in $\mathrm{B} 2 \mathrm{~B}$ markets.

Note 2. Reference is made to the data of the last available year at the time of the survey: 2012 .

Note 3. For instance: keywords related to the specific case studies analysed in the papers (ex. kajak industry).

\section{Copyrights}

Copyright for this article is retained by the author(s), with first publication rights granted to the journal.

This is an open-access article distributed under the terms and conditions of the Creative Commons Attribution license (http://creativecommons.org/licenses/by/3.0/). 